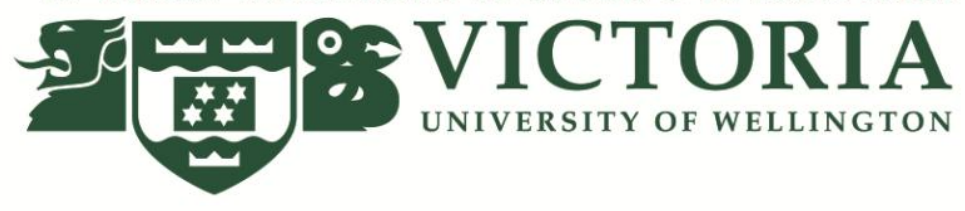

\title{
Factors and consumer attitudes that affect the sustainable management of used mobile phones: A repertory grid analysis.
}

By

Philip Coffey

A thesis submitted to the Victoria University of Wellington in fulfilment of the requirements for the degree of Master of Commerce in Information Systems

VICTORIA UNIVERSITY OF WELLINGTON 


\begin{abstract}
Used mobile phones with their small size but vast numbers, create a unique problem when it comes to managing the part they play in the creation of electronic waste (E-waste). Whilst previous studies have identified what consumers appear to be doing with their used mobile phones, there is also a need to better understand why they are doing it. This study investigated what factors appear to influence consumer attitudes towards adopting a more sustainable approach when dealing with their used mobile phones. A reuse, refurbish and recycle strategy was used as a lens to examine the current literature from which an initial model was developed. Using the repertory grid interview technique a group of participants was interviewed to try to determine their core beliefs when it came to managing their used mobile phones. Analysis of the interviews was completed using several analysis techniques including word clouds, percentage similarity analysis, and Honey's content analysis. The results of the study indicate that consumers care about the effect of used mobile phones on the environment although the degree of concern appears to vary across individuals. In addition, it was identified that in general, consumers perceive reuse, refurbishing, and recycling all as positive ways to sustainably manage used mobile phones, whilst environmental awareness appears to play a significant role in engaging people with recycling and being a rational for storing used mobile phones. Finally, the study suggests that telecommunication providers when trying to improve engagement with takeback schemes should focus more on consumers' environmental concerns and social norms, rather than financial incentives or promoting easy engagement.
\end{abstract}




\section{Contents}

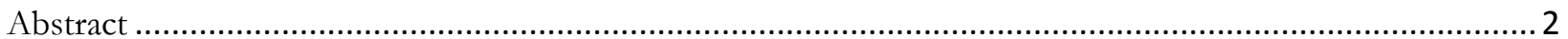

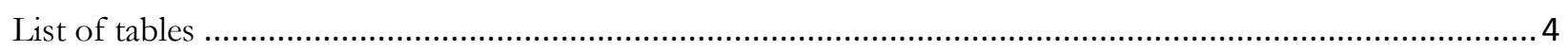

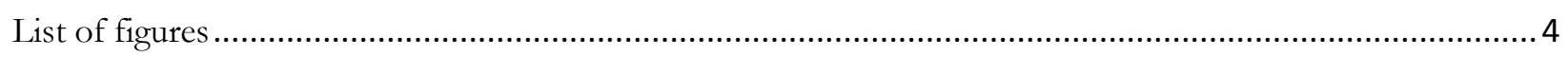

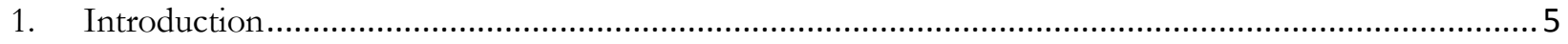

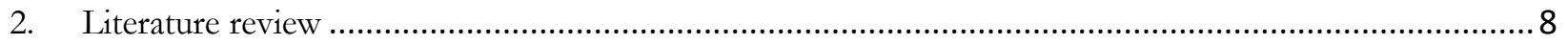

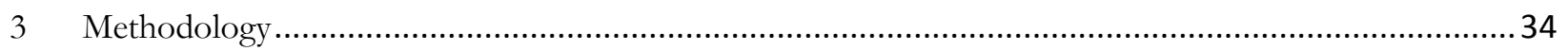

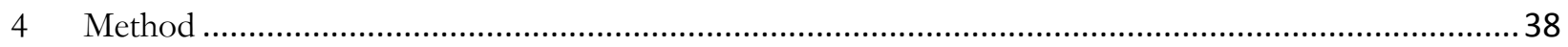

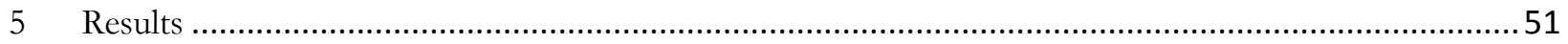

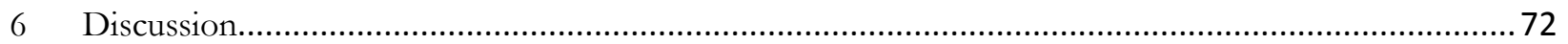

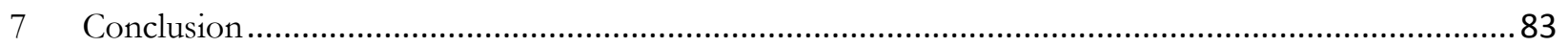

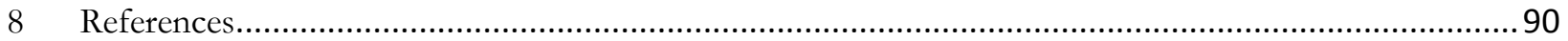

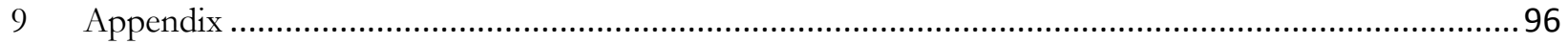




\section{List of tables}

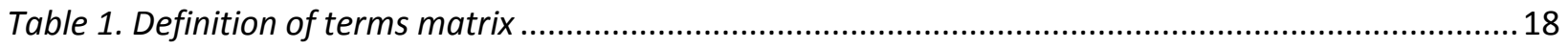

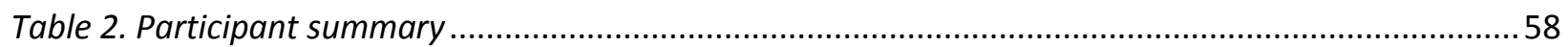

\section{List of figures}

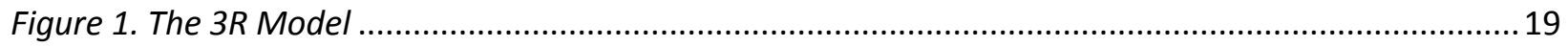

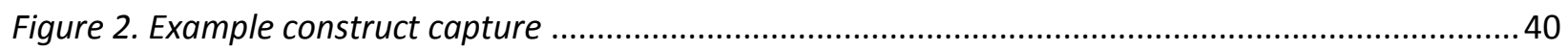

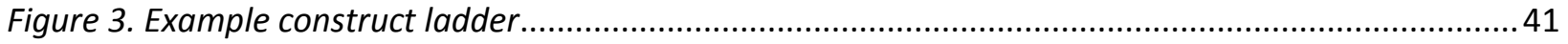

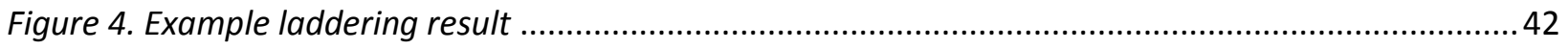

Figure 5. Example construct ladder with supplied construct - SUP ............................................................ 42

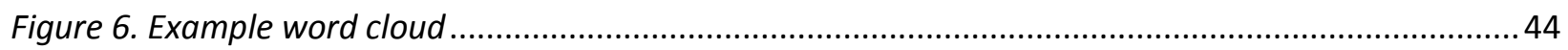

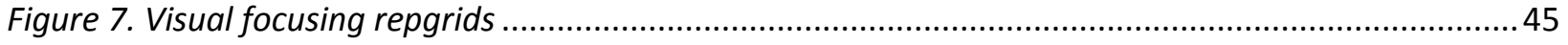

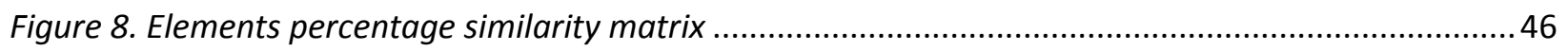

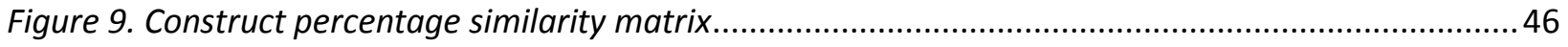

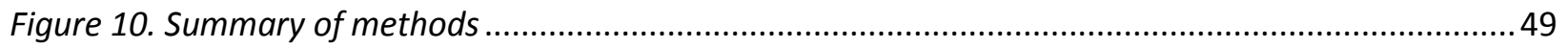

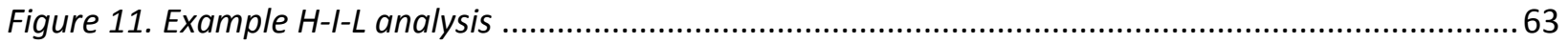

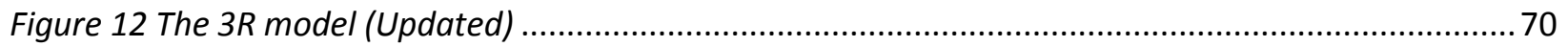

Figure 13. The $3 R$ model (Content analysis applied) ............................................................................... 71

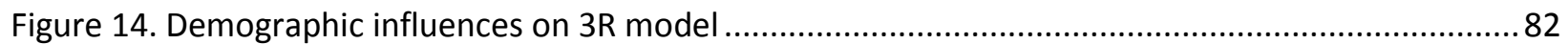




\section{Introduction}

\subsection{Research motivation}

As mobile phones become more widely used, society as a whole, shares responsibility for managing their environmental impact. This thesis attempts to explore this area, by trying to understand both the factors and consumer attitudes that play a role, however, as part of this process some assumptions have been made based on what would broadly be accepted as general knowledge. These assumptions include that as a planet, we are becoming more dependent on technology than at any other point in our history. This dependence on technology has both enriched our lives and created an expanding problem. Whilst the issue of electronic waste has been around since electronic consumer goods were first produced, as they have become more diverse and widespread, the issue of managing them when they reach the end of their useful lives has become increasingly problematic. A growing part of the electronic waste (e-waste) problem is the mobile phone. It is a ubiquitous device that has become an essential component of many peoples' lives, providing not only a way of keeping in contact with others, but also serving as a platform with which users can integrate many parts of their lives, from controlling home appliances to consuming entertainment such as games and movies.

The issue with mobile phones is that people appear to prefer to keep them rather than send them for recycling (Welfens, Nordmann, Seibt, \& Schmitt, 2013). The problem with this approach is that they are more likely to end up in the household waste stream than recycling centre's (Jang \& Kim, 2010). However, even when recycling options are available, it is suggested approximately $90 \%$ of mobile phones still end up in landfills (Silveira \& Chang, 2010). The problem with this outcome is that when mobiles phones are disposed of in landfills, rather than being recycled, there is the potential for them to leach toxic chemicals, such as heavy metals into the surrounding environment (Sarath, Bonda, Mohanty, \& Nayak, 2015). Even when mobile phones are recycled, if it is not done in a safe way, there is still potential to create significant environmental damage (Heacock, Kelly, \& Suk, 2016). This situation is made worse by the rapid life cycle of mobile phones that is between nine months and two years and is driven by both technological and fashion changes (Wilhelm, Hutchins, Mars, \& Benoit-Norris, 2015). 


\subsection{Research significance}

Because of the speed at which mobile phones are becoming obsolete, and new devices are being purchased, there needs to be an appreciation of the potential environmental impact. In order to help achieve this, research can attempt to explore how consumer attitudes and their underlying factors play a role when it comes to how consumers manage their used mobile phones. As a result, it is proposed that this research is significant because it attempts to explore these consumer attitudes, along with their underlying factors, and develop a model that explains their interaction. If research can better understand how these factors and attitudes work together, it may be possible to put into place systems and processes that will ensure more a sustainable outcome. This is important, for whilst sustainability should be balanced against societal progression, by ignoring the issues of small high-volume electronic waste, we run the risk of irrevocably changing or destroying vital ecosystems.

\subsection{Thesis structure}

\section{Chapter 1 -Introduction}

The current section introduces the research and the rationale for conducting it. It also provides a brief overview of the structure of the thesis.

\section{Chapter 2 -Literature review}

This section of the thesis examines the current body of knowledge in order to identify what consumer attitudes are potentially linked to the reuse, recycling and refurbishing of mobile phones. It also attempts to identify the underlying factors that may be driving those consumer attitudes. Using this information, a model is developed that attempts to explain these relationships.

\section{Chapter 3-Methodology}

In this section, various research perspectives are reviewed to help understand the rational for the perspective chosen in this research. It explores areas such as ontology and epistemology and discusses the specific methodology that was chosen. It also explores why the selected methodology is appropriate for the type of research being conducted.

\section{Chapter 4-Method}

Chapter 4 examines the underlying theory that that was adopted for this research, and how that theory forms the basis of the repertory grid (repgrid) and laddering interview techniques that were 
also used in this research. The chapter goes on to explain how these methods work together and how the repgrid method uses the concepts of reuse, refurbishing and recycling in conjunction with laddering to tap into the participant's view of the world. The various methods of analysis that were used in the research are also described, and finally a diagrammatic summary of the interview techniques and analysis methods is provided.

\section{Chapter 5 - Results}

In chapter 5 the results of the research are discussed, first from an individual participants' perspective, and then from a group perspective. For the individual participant analysis, demographic information is also included to give a sense of how participants' backgrounds may affect their views. At the end of the section, a summary is provided that contains both the participants' demographic information and a summary of the important areas that emerged from the analysis.

The group analysis section explores the results from a group perspective, and attempts to compare and contrast the results of each participant in order to identify similarities and differences based on the analysis of their responses. It goes on to present the results of Honey's content analysis, and uses those results to describe how adjustments were made to the original model, developed during the literature review. It also attempts to identify the relative importance of each of the factors and attitudes that are presented in the model.

\section{Chapter 6-Discussion}

In the discussion, the implications for the results are discussed with direct reference to both the factors and attitudes presented in the refined model. It compares the results to other studies and attempts to draw parallels with other researchers work. Finally, it attempts to discuss how the refined model fails to account for other potential factors, and how those factors may have an effect on the model presented.

\section{Chapter 7 - Conclusion}

In this section, conclusions are drawn and the implications for both theory and practice are discussed. The limitations of the research are also presented along with potential areas of exploration for future research. 


\section{Literature review}

The body of knowledge that examines how people interact with the natural environment and contains terms such as sustainability (Glavič \& Lukman, 2007) and electronic waste (e-waste) (STeP, 2016). Whilst these terms may be commonly referenced in everyday conversation about the environment, they still require a degree of exploration, to define what they mean in terms of this research.

\subsection{Sustainability}

The term sustainability is ubiquitous and has been used in a variety of contexts, and can relate to areas such as the economy or the environment (Brown, Hanson, Liverman, \& Merideth, 1987; Hart, 1997). Whilst the context may differ, it conveys a message of a self-supporting entity or system that works in harmony with surrounding entities and systems (Brown et al., 1987). Brown et al. (1987) attempted to establish a universal definition of sustainability with a focus on the interactions of humans with the wider environment. They defined that sustainability in its broadest sense includes the need to consider all of the components of a biosphere even when they have no readily identifiable benefit to people. For the purposes of this research, sustainability is defined as the need to manage any detrimental long-term environmental effects.

Although sustainability is a concept that has been around for a number of years there is a need to better understand both the factors and attitudes that are likely to impact an individual's adoption of sustainable practices (Kiron, Kruschwitz, Haanaes, \& Velken, 2012). A study by Leiserowitz, Kates, and Parris (2006) indicated there are three different types of barriers that exist when it comes to people adopting sustainability; these are global, individual and structural barriers. Global barriers are those values that limit the level of importance sustainability holds in comparison to rival ideas such as economic advancement. Leiserowitz et al. (2006) also discussed how barriers at the individual level are created because of limitations in areas such as knowledge, literacy and money. Whilst barriers such as knowledge and education can be addressed using educational programmes, financial inequity of individuals will always dictate the level of importance people place on adopting sustainable processes, especially if those processes have a financial impact. Finally, there are structural barriers, which are created by regulations, policies, and subsidies. Whilst policies exist that 
promote the use of less sustainable approaches, or where infrastructure is lacking, there will be only limited uptake of sustainable practices (Leiserowitz et al., 2006).

\subsection{E-waste}

The issue of electronic waste (e-waste) appears to be related to sustainability and has been defined as “......all types of electrical or electronic equipment (EEE) and its parts that have been discarded without intention for reuse by the owner" (STeP, 2016). For the purposes of this research, this definition of e-waste has been adopted.

Currently New Zealand does not have any legislation to deal with e-waste recycling (Morton, 2016). A report by written by the Computer Access NZ Trust (CANZ) that was the result of an investigation into computer e-waste in New Zealand identified that "The Government has made it clear that its preferred approach for dealing with e-waste is for voluntary industry-led solutions with shared responsibility." (MacGibbon \& Zwimpfer, 2006, p. 11). In 2008, the New Zealand government introduced The Waste Minimisation Act that created the ability to develop accreditation schemes and introduce legislation to help control for a broad range of factors related to waste disposal (Ministry for the Enviroment, 2015b). The Waste Minimisation Act 2008 focuses on all types of waste, not just electronic. It enables the Minister for the Environment to declare a priority product forcing that product to be managed through a mandatory product stewardship scheme. The Minster must be satisfied that three criteria are met before declaring a priority product;

1. The product will or may cause significant environmental harm when it becomes waste, or

2. There are significant benefits from reduction, reuse, recycling, recovery, or treatment of the product, and

3. The product can be effectively managed under a product stewardship scheme.

As yet, no products have been declared a priority product (Ministry for the Enviroment, 2015a).

The New Zealand Telecommunications Forum (TCF) is a registered incorporated society, which manages an accredited voluntary product stewardship scheme RE: MOBILE that encourages the recycling of mobile phones (New Zealand Telecommunications Forum, 2017). It has a number of members including some of the larger telecommunication providers in New Zealand, which include Vodafone, Telecom and 2Degrees. These telecommunication businesses provide collection bins at 
their mobile phone outlets in order to collect all types of mobile phones, along with their electronic accessories such as chargers (New Zealand Telecommunications Forum, 2017). Mobile phones that are collected and are still operational, are refurbished and sent for resale overseas, whilst phones that cannot be refurbished are broken down and sent to overseas recycling centers (Ministry for the Enviroment, 2014).

The existence of the TCF and its membership group is in line with suggestions made by Kiron et al. (2012) that some progress is being made towards sustainability from a business perspective, and that more businesses are adopting the view that sustainability is an important aspect of doing businesses. This increase in adoption appears to be driven by a growing consumer focus on sustainability (Kiron et al., 2012). Some advantages for businesses in adopting a more sustainable approach, is that they can end up reducing costs as they work with suppliers to develop more environmentally friendly materials (Nidumolu, Prahalad, \& Rangaswami, 2009) along with improving their processes (Russo \& Fouts, 1997). In addition, the affects of having a corporate image that is perceived to be associated with sustainability should not be underestimated. Whilst the link between corporate image, reputation and profitability is not always clear, it has been suggested that a focus on sustainability and environmental issues can lead to increased profitability, especially when sustainability is a concern for customers (Russo \& Fouts, 1997).

The European Union (EU) has also attempted to address the issue of e-waste by introducing the waste electrical and electronic equipment (WEEE) directive in 2003 which set the minimum levels of electronic waste that producers were required to collect (Silveira \& Chang, 2010). The EU also introduced the Restriction of Hazardous Substances (RoHS) directive, which came into force the same year, which restricted the amount of hazardous materials that could be used in electronic goods (European Commission, 2011). In 2013, an updated RoHS directive came into force (Department for Business Innovation \& Skills, 2014) and in 2014, a new version of the WEEE directive also came into force and included the introduction of a new measure to calculate the required amount of e-waste being collected (European Commission, 2016). According to the European Commission (2012a), this new measure was based on the average weight of electronic products that were introduced into the EU market over the previous three years and was to have been adopted by all member states by 2016. The new target was 45 percent of the average weight of WEEE that was produced across the European Union. This was to increase gradually until it reached 65 percent by 2019. Alternatively, member states had the option of adopting a measure of 
85 percent of the WEEE generated within their territory. Currently the EU predicts that 12 million tonnes of WEEE will be generated in 2020 in Europe alone (European Commission, 2016). The global amount of e-waste generated in 2012 was estimated to be 49 million tons and it has been projected that by 2017 e-waste will have increased to 65 million tons per year (STeP, 2013). The majority of e-waste can be attributed to rich nations (Panambunan-Ferse \& Breiter, 2013) with Perkins, Brune Drisse, Nxele, and Sly (2014) reporting that America generates nearly six times more e-waste than China per head of population.

\subsection{Mobile phones as e-waste}

Mobile phones vary in terms of their functionality, from the simple "dumb" phone, whose main function is voice and text messaging, to the high performance models that have the capability to run various applications. However, this research does not distinguish between these different types of mobile phones, their functionality, or the impact of that functionality on sustainability, but instead focuses on the mobile phone as a general concept.

As one of the smallest yet most widely used devices, mobile phones are a significant contributor to the issue of e-waste (Ongondo \& Williams, 2011b). Their contribution is becoming increasingly problematic as technological advancements lead to increasing numbers of mobile phones being released every year (Wilhelm, Yankov, \& Magee, 2011). The original brick phones available in the early 1980's were used by only a few people (Wilson, 2010), however, by 2013, mobile phone sales worldwide totaled 1.8 billion units, an increase of 3.5 percent from 2012 (Rivera \& van der Meulen, 2014). It is predicted that the number of mobile phones being retired every year will continue to grow, however, only a small number of these mobile phones are being collected (Geyer \& Blass, 2010).

In India, it is expected that the amount of e-waste created as a direct result of mobile phones will increase 18-fold by 2020 (Perkins et al., 2014), and in China it is estimated that about 70 million mobile phones are entering the e-waste stream every year (Wang, Zhang, Yin, \& Zhang, 2011). In Japan, it is currently estimated that there are over 200 million used mobile phones being stored in people's homes (Sugiyama, Honma, \& Mishima, 2016), whilst Ongondo and Williams (2011a) suggests that in the UK it is estimated that higher education students alone have 3.7 million phones stockpiled. When considering Europe, the total number of mobile phones being stockpiled is estimated to be 29.3 million, whilst in the USA there is estimated be 26.1 million (Ongondo \& 
Williams, 2011a). In New Zealand, in 2013/2014 there were over 5.3 million mobile connections with mobile revenue sales of 2.49 billion dollars, whilst it has been suggested that mobile phones are being replaced every 18 months (Commerce Commision, 2015). This results in estimates of up to three million mobile phones becoming obsolete every year with only about two per cent of those phones being recycled, with suggestions that over 70 percent of people have at least one unconnected mobile phone (Fletcher, 2014).

Mobile phones are now being designed to be more environmentally friendly, for example, Huawei have five mobile phones certified at platinum UL110 certification (Huawei, 2015). This certification was developed by UL, a multinational company, to measure the sustainability of mobile phones (UL, 2017). However, whilst progress has been made on making mobile phones more environmentally friendly, the issue of managing mobile phones once they reach the end of their useful lives remains problematic.

Mobile phones can be defined as being a disposal technology, that is, a technology that is expected to have a short life cycle (Huang \& Truong, 2008). The problem with mobile phones is that they are being created in large numbers, which is compounded by their rapid rate of succession, as new models are released (Wilhelm et al., 2011). This short innovation cycle along with low recycling rates means the issue of mobile phone waste will only increase (Perkins et al., 2014). Another problematic aspect of mobile phones is their relatively small size which means people find it easier to dispose of them in inconspicuous ways that are not necessary sustainable, such as in household rubbish (Huang \& Truong, 2008).

Their small size also makes them much easier to store, and as a result, consumers may be less inhibited when it comes to storing them in a cupboard or drawer because they take up little room (Baxter \& Gram-Hanssen, 2016). It may even encourage consumers to store several generations of mobile phones at home, compounding the problem for the future, as it only delays the entry of the mobile phones into the waste stream (Jang \& Kim, 2010). Because of their size, mobile phones are defined as category 6 under the latest waste electrical and electronic equipment (WEEE) directive, which includes all IT and telecommunication equipment that are no bigger than $50 \mathrm{~cm}$ in any direction. (European Commission, 2012b) 
Mobile phones that are collected for reuse often end up going to third world countries (Panambunan-Ferse \& Breiter, 2013), however, the importation of mobile phones for reuse can be problematic. For instance in 2010, 3,112,669 used mobiles phones were imported into Sri Lanka and that volume steadily increased to 3,968,084 in 2013, which indicates a 27 percent growth in import volumes over a 4-year period (Thavalingam \& Karunasena, 2016). This creates an issue when the mobile phones reach the end of their useful lives and need to be recycled, because in developing countries, the importation and recycling of e-waste is often unregulated (Heacock et al., 2015). Ghana for example has no regulations to manage the inflow of waste electronic products (AmoyawOsei et al., 2011). As a result, of the 70 percent of second hand products that were imported in 2009, a significant portion were deemed to be electronic products that were nearly at the end of their and likely to enter the waste stream within a short period (Amoyaw-Osei et al., 2011). Informal recycling is problematic because the recycling process, such as the burning of electronics in large open fires, releases large amounts of toxic gas (Sarath, Bonda, Mohanty, \& Nayak, 2015) and the process is often overseen by people without any protective clothing (Welfens et al., 2013). There is also the potential for chemicals within the mobile phones to combine during low temperature open burning and create by-products that are even more toxic than the phones individual components (Nnorom \& Osibanjo, 2009). Other environmental issues occur when toxic chemicals such as lead, mercury, chromium, plastics and flame-retardants leach into the surrounding environment (Heacock, Kelly, \& Suk, 2016). Some of the adverse health effects that have been shown to have a strong correlation with this type of e-waste pollution include spontaneous abortions, stillbirths, premature births and high levels of DNA damage (Grant et al., 2013).

The issue of informal recycling is also a problem in more developed countries where there is a lack of legislation. For example, in China acid baths and open incineration methods are still employed by private workshops (Yin, Gao, \& Xu, 2014). This type of practice has led to countries implementing legislation that prohibits the importation of used electronic waste. For instance Egypt has implemented legislation banning the import of electronic products that are intended for reuse if those products are greater than five years old (Chaplin \& Westervelt, 2015).

In 1994, New Zealand signed up to the Basel Convention, which deals with the import and export of hazardous waste across international boundaries (Ministry for the Environment, 2007). In 2002, the Basel Convention began to address issues of e-waste including introducing the Mobile Phone 
Partnership Initiative (MPPI), which promotes the management of end-of-life mobile phones and focuses on;

1. Achieving better product stewardship.

2. Influencing consumer behaviour towards more environmentally friendly actions.

3. Promoting the best reuse, refurbishing, material recovery, recycling and disposal options.

4. Mobilising political and institutional support for environmentally sound management. (Basel Convention, 2008)

In October 2008 the overall guidance for MPPI was submitted to the ninth meeting of the Conference of Parties to the Basel Convention and in October 2011 the final guidance for MPPI was adopted (Basel Convention, 2011).

\subsection{The 3 R's}

Various strategies have been proposed for dealing with e-waste, which has resulted in a variety of definitions (Rathore, Kota, \& Chakrabarti, 2011). Murugesan (2008) discussed a strategy for keeping e-waste out of landfills that consisted of three stages, reuse, refurbish and recycling (The 3 R's). This approach includes the option of reuse when products are still functional when they reach the end of their useful life with a particular consumer. A mobile phone may still be functional, but some owners feel the need to upgrade to a newer model every 1-2 years. The phone could be reused by being passed on to another consumer with more basic requirements.

Alternatively, King, Burgess, Ijomah, and McMahon (2006) suggest a strategy that consists of four stages, repair, recondition, remanufacture and recycling. Repairing, reconditioning and remanufacturing all relate to undertaking some degree of rework to prepare equipment for reuse. Recycling, however, occurs when all the previous approaches are no longer applicable. It is based on the idea of capturing raw materials from discarded waste through a process of breaking down the redundant equipment.

When it comes to the concept of refurbishing, the ideas presented by Murugesan (2008) appear to align more with King et al's (2006) repair, recondition, and remanufacture strategies. The intent appears to be the upgrading or reconditioning of technology, in order to extend its useful lifespan, rather than just replacing it with new technology. This extension of lifespan, as with reuse, aims to reduce the technology's overall environmental footprint. 
Both Murugesan's (2008) and King et al's (2006) option of recycling align clearly and describes the ultimate end-of-life cycle consideration for any technology. It comes after all other approaches for extending a products lifecycle have been exhausted. The importance of recycling is that it prevents electronic waste from ending up in landfills. This in turn prevents seepage of toxic chemicals into the local environment including waterways. Because the strategy discussed by Murugesan (2008) is more concise in nature, whilst capturing the essence of King et al.'s (2006) strategy, this study will adopt the 3 R's approach presented by Murugesan (2008).

\subsection{Understanding the 3 R's}

Whilst the 3R's proposed by Murugesan (2008) are strategies designed to limit the environmental impact of e-waste this study will consider the 3R's from a different perspective. The Collins online dictionary defines attitude as "the way a person views something or tends to behave towards it, often in an evaluative way" (HarperCollins, 2016). Using this definition, it is proposed that the 3R's can be used as a lens to examine consumer attitudes towards the sustainable management of used mobile phones. For this research, reuse, refurbish and recycle are all potential outcomes when a consumer's current mobile phone is replaced and is perceived to have reached the end of its initial useful life. However, each of these outcomes is based on a different underlying concept, the implications of which are discussed next.

\subsubsection{Reuse}

For the purposes in this research, reuse is defined as an outcome that is based on consumer attitudes towards the storing of a used mobile phone for future reuse, or alternatively, consumer attitudes towards the passing of a mobile phone onto a third party for reuse, but not recycling. This can include on giving or selling the mobile phone to friends, family, charity, or even being used as a trade-in for a new mobile phone. A mobile phone could even be stored for a period and then passed onto a third party.

\subsubsection{Refurbish}

Some studies (Quariguasi Frota Neto \& Bloemhof-Ruwaard, 2009) use the term refurbish or remanufacture to represent processes such as the repair or upgrading of a mobile phone for resale. However, for the purpose of this research, refurbishing is defined as an outcome that is based on consumer attitudes towards the purchasing of a refurbished mobile phone. 


\subsubsection{Recycle}

For the purposes in this research, recycling is defined as an outcome that is based on consumer attitudes towards engaging with recycling. For this study, this is when a mobile phone is sent to a third party to be broken down into its component parts or materials for reuse, but where the phone will not be reused as a communication device.

When it comes to managing e-waste recycling, the United States Enviromental Protection Agency (2012) identifies two conceptual approaches. The first is open loop recycling where the product at the end of the recycling process is often of a lower quality than the original material used. This results in the recycled material often being used in lower requirement products. An example, is where the plastics from PCs, when recycled, are often used in road projects rather than creating cases for new PCs. An alternative is closed loop recycling which is an approach that is ultimately more beneficial for the environment. To achieve this all recycled materials are able to be recycled back into the same kind of product. An example of this would be the process of recycling an aluminum can, and using the recycled material to create another aluminum can. This study does not differentiate between closed loop and open loop recycling, as they are both a more sustainable recycling approach, than dumping mobile phones in a landfill.

\subsection{Extending the 3R's - The 3R model}

A review of the current literature was undertaken using the library database available through Victoria University as well as the identification of relevant articles and books using the google scholar search engine. Keywords such as 'sustainability', 'mobile phones', 'attitudes' and 'e-waste' both individually and in combinations were used during keyword searches. Additional key words and sentences were then identified from the literature and used to expand the literature search further. Citation tracking was also used where citations in relevant articles were tracked back to their source material. The model (Figure 1) was developed using the 3R's described by Murugesan (2008) as a lens through which to examine the literature (Appendix 1). Since a significant body of knowledge had already been developed that examined mobile phones at the end of their useful lives from a sustainability aspect, it was anticipated there would be enough theory to develop an initial model (Braun \& Clarke, 2006). 
Initially, a causation, deductive coding approach was adopted during the literature review using methods described by Miles, Huberman, and Saldana (2013). Causation coding “...is appropriate for discerning motives, belief systems, world views, processes, recent histories, interrelationships, and the complexity of influences and effects on human actions and phenomena." (Miles et al., 2013, p. 79). This meant that during the literature review initial master codes were developed to reflect areas in each class of variable. For example, attitudes that affected reuse were coded as reuse-storing or reuse-on giving. The literature was then reviewed again to identify sub codes that reflected the model factors and their perceived direction of causation. However, it became clear that there was an overlap in terms of how certain factors or sub codes could be applied to more than one higher level attitude or code, as a result a simultaneous coding approach was then also adopted forming a hybrid approach. A simultaneous coding approach is where there is "...application of two or more different codes to a single qualitative datum, or the overlapped occurrence of two or more codes applied to sequential units of qualitative data."(Miles et al., 2013, p. 81). For example, when it was identified that "income" could relate to both "risk of refurbished" or "engaging" it was coded as both "income-risk refurb" and "income-engaging". This hybrid approach was adopted because it is sometimes necessary to development hybrid coding methods that reflect the needs of the study (Saldaña, 2013).

Whilst a number of relationships are proposed in this study, it is also likely there may be other interfactor relationships occurring that have yet to be identified. This means that whilst the model indicates absolute relationships in order to maintain clarity of presentation, it is possible that the factors themselves have influential relationships with each another. Therefore, it is possible groups of factors will have varying levels of influence across the system depending on the users unique set of life experiences. It should also be noted that whilst the link between people's attitudes and behavior has been investigated by a number of researchers, it is not within the scope of this research.

Finally, for the purposes of this study, several terms can represent the same meaning (Table 1). This means that in some places, the terms may have been used interchangeably depending on whether the 3R model was being discussed or the results of the content analysis coding were being discussed. 


\begin{tabular}{|c|c|c|}
\hline Model & Coding & Example \\
\hline Outcome & Theme & Reuse, Refurbish, Recycle \\
\hline Attitude & Category & Storing, On giving, Purchasing refurbished, Engagement \\
\hline Factor & Subcategory & Size, Spare, Ease, Environmental awareness.... \\
\hline
\end{tabular}

Table 1. Definition of terms matrix 


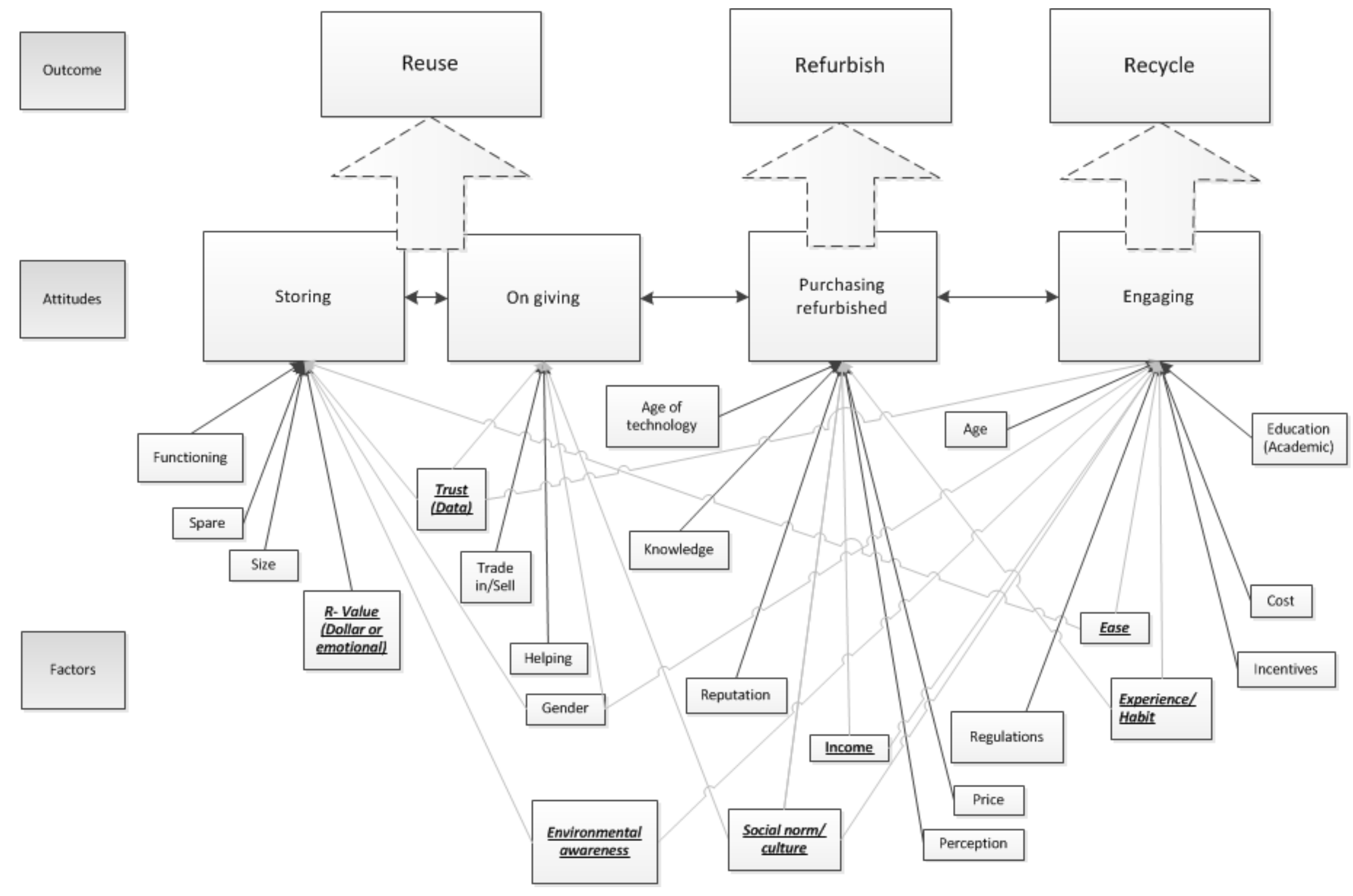

Figure 1. The 3R Model 


\subsubsection{Reuse}

During the literature review two attitudes were identified as affecting reuse, these were storing and the on giving of mobile phones. These attitudes are in turn influenced by a number of underlying factors, these attitudes and underlying factors are discussed next.

\section{Storing (Attitude)}

Once a mobile phone reaches the perceived end of its useful life several researchers have identified that they are often stored (Baxter \& Gram-Hanssen, 2016; Ongondo \& Williams, 2011a; Speake \& Yangke, 2015; Suckling \& Lee, 2015; Welfens et al., 2013; Wilhelm et al., 2015; Ylä-Mella, Keiski, \& Pongrácz, 2015). The term storing for this research, is defined as the attitude a person has towards storing a mobile phone. This attitude can be influenced by several types of factors, including whether it is intended the mobile phone is to be used in future or whether to keep the mobile phone for purely sentimental reasons. For the purposes of this study, the storage location of the mobile phone whether it is in a drawer at home or elsewhere is less important than the intent behind the reason to store it.

\section{Gender (Factor)}

The impact of gender on the storage of mobile phones was examined by Ongondo and Williams (2011a) and it was suggested there was a link between gender and mobile phone storage. They concluded that more females store mobile phones when the number of mobile phones being stored was between one and two. Conversely, more males stored mobile phones when the number of mobile phones being stored was greater than three. Overall, it was concluded that in total males appeared to be storing more mobile phones than females. One of the reasons suggested to explain this was that females might be more attached to their phones whilst males may be prefers to replace them more often.

\section{Spare (Factor)}

The notion of storing a spare phone has been linked to the perception of needing a spare phone, if the users' new phone becomes lost or stops working (Welfens et al., 2013). However, other studies identified that mobile phones are stored even if they are no longer working (Huang \& Truong, 2008; Ylä-Mella et al., 2015) which means they have no use as a spare unless they are deemed useful for spare parts. It has also been suggested that even 
when a phone is stored with the intent of future use, users rarely actually end up reusing their spare backup phone (Huang \& Truong, 2008). The act of storing a mobile phone as a spare precludes it from realising its full reuse potential (Ylä-Mella et al., 2015).

\section{Functioning (Factor)}

As already mentioned, whilst consumers often keep a phone as a backup (Huang \& Truong, 2008) there is an assumption that the phone is in working order. However, in a study by Welfens et al. (2013) it was identified that over 55 percent of the respondents not only kept working mobile phones but also mobile phones that were no longer functioning. This conflicts with the idea that a mobile phone is stored for future use, and instead suggests some other drivers may also be at play.

\section{Residual value - Financial or emotional (Factor)}

Mobile phones may still have some economic residual value (Thavalingam \& Karunasena, 2016) or residual emotional value (Welfens et al., 2015; Ylä-Mella et al., 2015) once they are perceived to have reached the end of their useful lives. The economic value that remains locked within a mobile phone may be based on just the raw materials or could be the brand and level of functionality attributed to a top range phone that is still in demand. The residual emotional value a user may attach to their mobile phone may occur because of how pervasive mobile phones are in many aspects of our everyday lives (Welfens et al., 2015). If

some users perceive their old mobile phones have residual value they may feel opposed to just disposing of it or giving it away (Baxter \& Gram-Hanssen, 2016; Ongondo \& Williams, 2011a). With no simple and immediate alternatives to recovering the perceived residual economic value or rationalising away the emotional value, users may resort to storing the phone.

\section{Size (Factor)}

Studies that have examined e-waste, suggest that larger items such as fridge's and televisions are more difficult to dispose of in an inconspicuous manner because their size inhibits irresponsible disposal methods, their size also means they are less likely to be stored (Baxter \& Gram-Hanssen, 2016; Darby \& Obara, 2005). However, mobile phones are small and do not face the same size barriers associated with larger e-waste items, which means that a 
mobile phones small size, may encourage both storage as well disposal in general house waste (Baxter \& Gram-Hanssen, 2016).

\section{Ease (Factor)}

Effort has been identified as an important factor in influencing a user's decision to store their old phones, as effort is often required in not only seeking out an environmentally friendly disposal options, but also to deliver the used mobile phone either by hand or by mail (Thavalingam \& Karunasena, 2016; Ylä-Mella et al., 2015).

\section{Environmental awareness (Factor)}

A lack of environmental awareness has been suggested to play a role in why old mobile phones are stored, that is because users may perceive that storing their old mobile phone is an environmentally neutral approach (Baxter \& Gram-Hanssen, 2016). Unfortunately stored mobile phones are thought to be more at risk of being disposed of in general household rubbish (Jang \& Kim, 2010), and often because they are stored for so long they can become obsolete, which hinders the ability to extend their useful lives (Welfens et al., 2013).

\section{Trust (Data) (Factor)}

Welfens et al. (2015) suggested that the issue of trust might be linked to users preferring to store their old mobile phones. The trust issue relates to the lack of transparency that may surround recycling processes and the fear that unscrupulous recycling providers may try to leverage some value from any data that remains on an old mobile phone once it is sent for processing

\section{On giving (Attitude)}

For this research, on giving is defined as the on giving or selling of a mobile, whether that be family, friends or even using the phone as a trade-in to purchase a new mobile phone. The ultimate intent of this decision is that the mobile phone will be reused in some fashion.

\section{Helping (Factor)}

In deciding whether or not to pass on an old mobile phone users have the ability to assess the impact and outcome of their actions. Some studies have identified that users may give away their old mobile phones to either family members or friends (Ongondo \& Williams, 
2011a; Welfens et al., 2015; Yin et al., 2014). Since there does not appear to be an economic incentive to their actions, it must be assumed the phones are being passed on to be helpful, and the reward may be based on the strengthening of social relationships.

\section{Trade in/Sell (Factor)}

Users have several options when looking at disposing of their old mobile phone. However, it has been suggested users are unlikely to dispose of their old mobile phones through recycling schemes when they are able to reclaim some of the residual economic value by selling them (Yin et al., 2014). If harnessing economic value were important then it would likely also apply to users who decide to trade in their old phones when they upgrade (Welfens et al., 2013). Contract renewals that encourage mobile phone trade-ins are likely to incentivise users to trade in their old mobile phone and maximize any residual value (Huang \& Truong, 2008).

\section{Social Norm/ Culture (Factor)}

In general, it has been suggested that behaviour is impacted by both the social and cultural background of individuals (Hansmann, Bernasconi, Smieszek, Loukopoulos, \& Scholz, 2006) which may help explain why users do not always pass on old mobile phones to family and friends. In this regard, a study by Panambunan-Ferse and Breiter (2013) suggested users are less likely to pass on their mobile phones if they are perceived to be old and outdated. This is because giving away old technology as gifts is not perceived in a positive way by the wider social community. This means, that whilst some users do pass on their old mobile phones because they may want to help, the decision to do so may be affected by social or cultural perceptions.

\section{Trust (Data) (Factor)}

Whilst trust may play a role in whether users decide to store their old mobile phones (Welfens et al., 2015) it appears the same concerns may also play a role in a user's decision to pass it onto a third party. Kissling et al. (2013) identified that secure data sanitisation is a barrier for users who considered engaging in the reuse of electronic equipment, and indicates users are concerned that their personal information may be used without their permission. However, this does not mean that an increased level of trust in a mobile phone take back 
program will lead to a proportional decrease in mobile phones being stored, and a proportional increase in the number of phones being sent for processing. It is suggested; that the degree of influence trust has on the decision to store or give to a third party will be different for each user.

\section{Gender (Factor)}

As with storing a mobile phone, gender has also been indicated as potentially having an impact on a consumer's attitude towards on giving a mobile phone to a third party. This is because Darby and Obara (2005) suggested females are more likely to give their old mobile phones to charity compared to males.

\subsubsection{Refurbish}

Refurbishing has been linked to one underlying attitude, the attitude towards purchasing a refurbished mobile phone. That is because the process of sending a phone away to a third party for refurbishing is already contained within the reuse outcome, whilst there is still a need to account for people's perceptions towards purchasing a refurbished mobile phone.

\section{Purchasing refurbished (Attitude)}

As with attitudes towards storing and on giving, it appears purchasing, a refurbished mobile phone is impacted by a number of underlying factors.

\section{Knowledge (Factor)}

In making the decision to purchase a refurbished mobile phone, it is suggested that knowledge of how mobile phones are refurbished, may help users balance the perceived benefits and risks associated with purchasing used goods (van Weelden, Mugge, \& Bakker, 2015). It has been proposed, that some degree of knowledge assurance could be achieved by the use of a quality verification process (Ylä-Mella et al., 2015), as quality has been identified as a major concern amongst potential buyers (Kissling et al., 2013). Knowledge could also be increased by promoting and using more transparent refurbishing processes, including the ability to track mobile phones from their source (Kissling et al., 2013). This approach might also help combat issues where users feel phones have been designed to break down after a certain period of time (van Weelden et al., 2015), however, media coverage of the predicted 
life expectancies of popular brands is likely to impact the issue of perceived design obsolescence (Gibbs, 2016).

\section{Reputation (Factor)}

If all stakeholders in the refurbishing value chain have a strong reputation, this helps users with managing their risk assessment of purchasing used goods (Kissling et al., 2013), and is important where users may have suspicions around reliability (Ylä-Mella et al., 2015), which also indicates brand reputation may play a role in consumer choice (Geyer \& Blass, 2010). However, Abbey, Meloy, Guide, and Atalay (2015) have suggested there may be complex links between brand and the decision to purchase reused goods, as a result, it is not possible to fully understand how concepts such as brand, influences intent to purchase with our current understanding (van Weelden et al., 2015). It has also been suggested that second hand dealers only play a minor role in the overall mobile phone market (Jang \& Kim, 2010), however, this only appears to apply to more developed countries. In third world countries second hand mobile phones dealers have a greater share of the mobile phone market, which is indicated by the need to import second hand phones to keep up with market demand (Thavalingam \& Karunasena, 2016), and is also supported by Apple's attempt to enter the second hand phone market in countries like India (George, 2016).

\section{Price (Factor)}

Several factors have been established as likely to affect the resale value of used mobile phone, these include brand, model, age and condition (Geyer \& Blass, 2010). Studies by YläMella et al. (2015) and van Weelden et al. (2015) identified that price is a leading factor in the decision to purchase a used mobile, this idea is further explored by van Weelden et al. (2015), where it was suggested product price has a twofold impact. Too high a price will likely result in users not purchasing a used mobile phone because it may not be seen as value for money. On the other hand, users are also likely to reject a used mobile phone if it is priced too low, as it introduces suspicions around quality. In contrast, and as with reputation, it has been suggested that whilst price does appear to play a role, how it actually impacts a user's decision to purchase a used mobile phone in the context of all other factors is not clear (Abbey, Meloy, Guide, et al., 2015). 
Age of technology (Factor)

The fast pace of technology can result in the perception that products have shortened lifespans which is a concern for some consumers (Ylä-Mella et al., 2015), as it can create the perception that a second hand mobile phone will quickly become obsolete (van Weelden et al., 2015). As a result, it has been suggested that within an eight month period some mobile phone models can lose up to 30 percent of their resale value (Geyer \& Blass, 2010). This may deter some users from purchasing a refurbished mobile phone.

\section{Income (Factor)}

The demand to own a mobile phone is ubiquitous but is likely hindered by consumers' income levels. This economic barrier to owning a mobile phone is consistent with the suggestion that the financial status of people has an impact on the decision to purchase a second hand mobile phone (Thavalingam \& Karunasena, 2016). If the financial status of an individual affects their ability to purchase, it is more likely that people with low incomes are more likely to use second hand mobile phones (Wang et al., 2011).

\section{Perception (Factor)}

An individual's perception of the acceptability of purchasing a second hand mobile phone is likely to affect their attitude towards refurbished mobile phones. This is supported in a study by Abbey, Meloy, Blackburn, and Guide (2015) where free association was used to determine consumer perspectives of refurbished products. One of the effects consumers demonstrated was that of repulsion or disgust. If a consumer feels a sense of repulsion or disgust towards a refurbished mobile phone, that sense of aversion is likely to affect their attitude towards purchasing a refurbished phone. In fact, it has been show that reminders of the refurbishing process can actually increase levels of aversion (Abbey, Meloy, Blackburn, et al., 2015). It has also been suggested, that refurbished products do not give consumers the same degree of purchase enjoyment as new products (van Weelden et al., 2015).

\section{Social Norm/Culture (Factor)}

As previously suggested, social and cultural background may affect behaviour (Hansmann et al., 2006). Therefore, a person's attitude towards the use of a refurbished mobile phone can be affected by their social referents as people rely on other people's opinions when making decisions (van Weelden et al., 2015). This means that through social influence those around 
us are able to influence the way we perceive and develop our attitudes, which in turn means we can also influence those around us. For example, it has been identified that family has a significant impact on a person's perceptions of waste (Ojeda-Benítez, Armijo-de Vega, \& Marquez-Montenegro, 2008). If a person belongs to a social group that actively encouraged the use of refurbished mobile phones through positive affirmation, it is possible that their opinion of using refurbished mobile phones would also be positive. However, the same effect would also hold true for a group that held negative views towards refurbished mobile phones. This means that if perceptions around mobile phone reuse need to be addressed as part of an educational program, groups of individuals would need to be targeted so they can understand the sustainability impacts of the choices they make (Welfens et al., 2013).

\section{Experience/Habit (Factor)}

It has been suggested that habitual behavior towards ignoring refurbished products means consumers are less likely to consider refurbished goods especially when they are not offered by standard retail outlets (van Weelden et al., 2015). This suggests that consumers may be more likely to purchase a new mobile phone by force of habit than consider the option of purchasing a refurbished mobile phone.

\subsubsection{Recycle}

Recycling is the last of the three outcomes presented in the $3 \mathrm{R}$ model, and as with refurbishing, has been linked to a single attitude, engaging.

\section{Engaging (Attitude)}

Engagement is defined as the decision consumers make to engage in the recycling of mobile phones. It is proposed that engagement is directly affected by several underlying factors. Engagement is important, because for any recycling program to be successful, consumers need to be actively engaged (Yau, 2012).

\section{Regulations (Factor)}

From a consumer perspective a low level of environmental engagement appears to be associated with the absence of regulations (Yin et al., 2014), and it is suggested that any mobile phone take back scheme needs to include some mandatory aspects (Silveira \& Chang, 2010). The issue of regulations and the role they play in achieving a greater degree of 
environmental engagement, is also raised by several other studies (Baxter \& Gram-Hanssen, 2016; Jang \& Kim, 2010; Speake \& Yangke, 2015; Suckling \& Lee, 2015; Thavalingam \& Karunasena, 2016). It is important to consider though, that whilst more developed nations such as those found in the EU use regulations as a tool for to helping manage engagement, less developed countries tend to have fewer regulations when it comes to e-waste (Nnorom, Ohakwe, \& Osibanjo, 2009). This does pose a problem since most refurbished mobile phones are sold in developing countries (Geyer \& Blass, 2010)

\section{Ease (Factor)}

When it comes to engaging with mobile take back programmes, the collection method and the overall convenience of using the program, is a factor in whether or not consumers use the program (Ongondo \& Williams, 2011a, 2011b; Welfens et al., 2015). This includes having permanent (Ylä-Mella et al., 2015), easily accessible and highly visible return points located in high traffic public and private places (Silveira \& Chang, 2010). The ready accessibility of return points decreases the difficulty of engaging with return programmes and allows consumers to more easily adopt the use of a return program into their everyday lives (Huang \& Truong, 2008). Conversely, if programmes are time intensive and lack convenience, the degree of consumer engagement decreases (Nnorom et al., 2009; Wang et al., 2011).

\section{Incentives (Factor)}

Incentives are deemed important in getting consumers to engage in take back programmes (Ongondo \& Williams, 2011a) and the absence of any incentives is suggested to be a barrier to people returning old mobile phones (Wilhelm et al., 2015). Incentives need to be in the form of "immediate material compensation" (Welfens et al., 2015, p. 5) in order to engage consumers and previous efforts have included monetary payments and donations to charity (Ongondo \& Williams, 2011b). It is also possible for incentives to have a perverse effect, particularly when network operators offer new phones or free upgrades, these are unlikely to be declined and therefore speed up the rate at which new technology becomes obsolete (Huang \& Truong, 2008; Ongondo \& Williams, 2011b). However, a study conducted by Speake and Yangke (2015), suggested there was little evidence to indicate incentives motivated consumers to engage with take back programmes. 
Cost (Factor)

Cost can be used to describe any impact related to time or finance. For this study, the impact of time is included under the ease of disposal, and therefore cost for this study is purely financial. As a result if the financial cost of engagement remains low consumers are more likely to engage in take back programmes (Huang \& Truong, 2008; Wilhelm et al., 2015), however, consumers may be more willing to pay when there are regulations involved (Wang et al., 2011).

\section{Social Norm/ Culture (Factor)}

Supported by previous assertions that social and cultural background may affect behaviour (Hansmann et al., 2006), the presence of social norms and culture can act as a driver to encourage engagement, or as barrier, that limits engagement (Thavalingam \& Karunasena, 2016; Welfens et al., 2015). This is because people tend to refer to the beliefs of others when evaluating choices (van Weelden et al., 2015). It also means that those with strong beliefs, for instance, what is good for society may have an influence on those around them which is supported by Hansmann et al. (2006) who suggested that behaviour is impacted by both the social and cultrual background of individuals. However, the absence of social norms and culture in relation to recycling can create a barrier to engagement (Wilhelm et al., 2015) which suggests that the default position of consumers when there are no social or culture pressures, may be disengagement.

\section{Trust (Data) (Factor)}

A lack of trust has been suggested to play a role in the formation of attitudes towards reuse, and the same factors appear to influence consumers' choice when it comes to sending phones for recycling. Mistrust appears to occur when there is a lack of transparency around the collection process and can create a level of concern around the inappropriate use of information that may remain on the phone once it has been returned (Welfens et al., 2015; Welfens et al., 2013). However, a study by Baxter and Gram-Hanssen (2016) suggested that concerns around information security did not appear to be a major concern in household surveys and as a result may be more of a concern for businesses. 


\section{Income (Factor)}

It has been suggested that consumers with higher income levels are more likely to engage with take back programmes (Nnorom et al., 2009), which is supported by Wang et al. (2011) who suggested low income is a barrier to engagement, and as a result low income people rarely engage with recycling programmes. One reason behind this suggestion is that low income people may not be able to afford the transport to go to recycling centres. Another explanation may be that people with higher incomes place less emphasis on the potential residual financial value of their old phones. Contrary to the low-income barrier suggestion, Wang et al. (2011) in a study of Chinese consumers suggested there was no difference in the willingness of poor or wealthy consumers to pay for recycling. This idea was extended further in a study by Zhao, Gao, Wu, Wang, and Zhu (2014) that suggested consumers with higher incomes were less likely to take part in recycling programmes.

\section{Gender (Factor)}

Several studies have indicated that there appears to be a difference in the level of engagement displayed by each gender when it comes to recycling (Darby \& Obara, 2005; Speake \& Yangke, 2015). Both Speake and Yangke (2015) and Darby and Obara (2005) suggest that females were either more engaged or more willing to recycle compared to males, however, the increased level of female recycling only appeared to occur when they were aware of recycling options (Darby \& Obara, 2005).

\section{Age (Factor)}

An examination of the impact of age on environmental awareness indicated a link between age and the awareness of mobile phone take back schemes, and showed that students aged 24 years or younger, were more aware of available phone take back schemes than older students (Ongondo \& Williams, 2011a). This potential lack of environmental awareness amongst older people aligns to some degree with a study by Nnorom et al. (2009), which showed people older than 65 years of age were less willing to pay a premium on mobile phones to ensure mobile phones were disposed of in an environmentally sustainable way. It is important to note, that the authors of the study pointed out that older people may be less swayed by additional features of new phones, of which being environmentally friendly may be a part. 
Other studies have suggested age plays a role in how engaged people are for different reasons (Speake \& Yangke, 2015; Wang et al., 2011; Zhao et al., 2014). These studies propose that older people are more likely to participate in recycling programmes when compared to younger people because older people have experienced the depression era (Zhao et al., 2014). Whilst Ongondo and Williams (2011a) suggests a correlation does exist between age and willingness to recycle, the study used university students as its sample group, which means it is unlikely they were exposed to depression era experiences. However, this does not preclude the idea that other difficult economic times may have shaped their experiences. Other studies have indicated that the younger millennial generation is one of the most socially conscious generations (Meister \& Willyerd, 2010) with concern for both environmental and social issues (Barber, Taylor, \& Dodd, 2009).

\section{Education - Academic (Factor)}

It has been suggested that more highly educated consumers are able to better understand complex environmental issues (Zhao et al., 2014) and are therefore more likely to engage in recycling. However, when academic education was tested there did not appear to be a significant difference in environmental awareness between consumers who had an undergraduate or postgraduate degree. There was a difference noted though, when comparing college graduates to consumers who only had a high school qualification or less (Yin et al., 2014). Therefore, it appears as though the difference may be most pronounced when comparing consumers who have tertiary qualifications, with those that do not.

\section{Environmental awareness (Factor)}

Of all potential factors, environmental awareness appears to be one of the most important. In this study, environmental awareness includes education around take back services for both reusing and recycling mobile phones. It has been linked to consumers being willing to pay for more environmentally green electronic products (Nnorom et al., 2009), whilst a lack of environmental awareness has been associated with consumers not engaging with take back programmes (Ongondo \& Williams, 2011a, 2011b; Welfens et al., 2015). This means consumers are more likely to dispose of mobile phones in an environmentally unsustainable manner (Thavalingam \& Karunasena, 2016) and it has been suggested that consumers who 
have a greater environmental awareness are more likely to engage in recycling compared to those who are environmentally unaware (Leiserowitz et al., 2006; Welfens et al., 2015; Yin et al., 2014). It has been proposed that when trying to raise environmental awareness through education messages those messages should be clear (Welfens et al., 2013) and should be delivered in ways that target specific social groups (Welfens et al., 2015). However, Wang et al. (2011) suggested that environmental awareness was not a statistically significant factor in consumers recycling behavior, which is similar to a study by Baxter and Gram-Hanssen (2016) that suggested even when consumers are environmentally aware they are less likely to recycle mobile phones as intensively as they do other types of electronic products.

\section{Experience/Habit (Factor)}

It has been proposed that having had some level of experience with general recycling to the point of creating a habit is linked with e-waste recycling (Speake \& Yangke, 2015). This suggestion is based on the idea that the habit of recycling non e-waste products would have become ingrained in a person's everyday behavior. As a result, the step to recycling e-waste does not require the establishment of new habits and it is merely an extension of current habits (Welfens et al., 2015). This is important because the creation of new habits can be extremely difficult (Darby \& Obara, 2005).

\section{Gender (Factor)}

It has been suggested that there is a gender difference between males and females when it comes to engagement (Speake \& Yangke, 2015). The proposed difference is based on the results of testing whether different genders have a different willingness to pay when it comes to environmentally friendly phones. The results indicated females were more willing to pay and as a result, it was proposed they might have a greater environmental consciousness or awareness.

\section{Summary}

The purpose of the literature review was to examine what research had been done that related to the sustainable management of used mobile phones and to identify potential underlying factors and consumer attitudes. Because reuse, recycle and refurbish have been suggested as a strategy to encourage managing used IT products in more sustainable way, the literature was examined using 
these three areas as focal points. The resulting $3 \mathrm{R}$ model (Figure 1) has attempted to explain the relevance of each factor and its relationship to higher-level attitudes as a first step in attempting to answer the question,

What factors and consumer attitudes affect the sustainable management of used mobile phones? 


\section{Methodology}

\subsection{Paradigm}

When it comes to information systems (IS) the more traditional natural science approaches have historically formed the basis of mainstream research, with the adoption of interpretive research being a more recent occurrence. An indication of its growing acceptance was when MIS quarterly promoted its adoption in 1993 (Walsham, 1995a). Interpretive research is based on the perspective that "....our knowledge of reality is a social construction by human actors" (Walsham, 1995a, p. 365). Both Myers (2013) interpretive paradigm and Guba and Lincoln's (1994) critical theory and constructivism paradigms accept the subjective nature of research and acknowledge that participant values are an important part of any research. Whilst these three subjectively based paradigms are related, Guba and Lincoln (1994) propose critical theory focuses on both the historical realism of the environment and the ability to make change within that environment. This research is less focused on the historical context or attempting to make any changes to what is being studied; it is focused on understanding how participants construct their own realities. As a result the interpretive paradigm suggested by Myers (2013) and the constructivism paradigm suggested by Guba and Lincoln (1994) appear consistent with the intent of this research. A further examination of these paradigms suggests that while they have different titles, they have similar philosophical perspectives. However, Myers (2013) interpretative paradigm resonates more deeply with perspective of this research and is ultimately, why it was adopted. It is important to note though, that whilst a paradigm reflects the researchers view towards data and its treatment, the selection of both the data collection and data analysis techniques are not directly linked to the choice of paradigm (Sandelowski, 2000).

\subsection{Qualitative research}

The decision on which approach is appropriate is driven by the purpose of the research. Quantitative methods are based in the natural sciences; they rely on the generation of numerical data that allows for mathematical modeling and statistical analysis. Qualitative methods on the other hand are based in the social sciences and rely on rich descriptive data generated whilst studying social phenomenon (Myers \& Avison, 2002). This research is focused on understanding socially based phenomena; as a result, a qualitative method has been adopted. This is supported by Myers (2013) 
who argues that if you want to investigate what motivates people and the reasons for what they do along with context of their beliefs, qualitative research is the most appropriate approach.

\subsection{Ontology}

The ontological perspective adopted by a researcher is based on what perspective that researcher holds of both the social and physical worlds (Myers \& Avison, 2002). There is the objective worldview where the world is seen to exist independently of humans and so can be measured empirically and can be understood from an objective perspective. There is also the subjective perspective, where the world is viewed as being created by, and only existing because of the actions and interactions of the people within that world. The interpretive researcher recognises the world is socially constructed and it is the action and interaction of people that reinforces the constructs that create the world. As a result, it is impossible for the interpretive researcher to view the world from a purely objective perspective (Myers \& Avison, 2002).

Archer (1988) suggested three ontological perspectives, 'external realism' where reality is constructed independently of people's actions, 'internal realism' where reality is created between people and the related human experience and 'subjective realism' where each person is responsible for how they construct their own reality. Usually an IS researcher undertaking interpretative research would be aligned with either 'internal realism’ or ‘subjective realism’ (Myers \& Avison, 2002).

By adopting a subjective perspective, researchers acknowledge their own biases and preconceptions will have an effect on the research. It is also important to acknowledge that the researcher's interaction with the subjects changes both the perception of the researcher and that of the subject (Walsham, 1995a). The 'internal realism’ postulated by Archer (1988) and suggested in Myers and Avison (2002) for interpretive research will be adopted for this research. That is because whilst the subjective nature of the world is important it must be considered in the context of the experiences people create when they interact with one another.

\subsection{Epistemology}

Epistemology defines what is needed to both construct and evaluate knowledge (Myers \& Avison, 2002). Since this study recognises the importance of the subjective nature of the social world it means the social world cannot be understood using hypothetical deductions or empirical analysis. It 
requires an understanding of the meanings of social practices and how they are shared and understood between people (Myers \& Avison, 2002). This means in order to understand what has occurred the researcher needs to understand the meaning behind what has occurred. That is because only by understanding the intent behind the action can you understand why the action was being undertaken. Understanding of the intent can only be realised when the social system with its intrinsic meanings is also considered (Guba \& Lincoln, 1994).

Archer (1988) suggests two epistemological perspectives for the interpretive researcher, the nonpositivism perspective, or the normative perspective. The non-positivism perspective is that "Facts and values are intertwined and hard to disentangle: both are involved in scientific knowledge" whilst the normative perspective is where "Scientific knowledge is ideological and inevitably conductive to particular sets of social ends" (Archer, 1988, p. 273). This research rejects the ideological nature of scientific knowledge and accepts that knowledge is a complex mix of facts and values, which cannot be easily disengaged from each other (Walsham, 1995b).

This research is attempting to understand people's perspective of the world when they think of sustainable mobile phone practices. It is attempting to achieve this by understanding the underlying factors that drive their attitudes, which ultimately influence their actions. This can only be achieved when their attitudes are considered in the context of their social environment, as the environment will attribute meaning to their attitudes. Therefore, this study is adopting a non-positivism epistemological perspective.

\subsection{Methodology}

Many research articles promote the use of an interpretive approach without defining what that means (Caelli, Ray, \& Mill, 2003). This includes the inability to clearly state the underlying epistemological, ontological and methodological perspectives. Whilst this research adopts an interpretive approach, and has identified its ontological and epistemological perspectives, the classical methodologies attributed to the interpretive paradigm such as a case study, ethnography or grounded research do not succinctly fit this research. As a result this research will adopt a pluralistic approach (Landry \& Banville, 1992) and draw on a methodology that has been used extensively in health science research (Brewer, Harwood, McCann, Crengle, \& Worrall, 2014; Kalengayi, Hurtig, Ahlm, \& Ahlberg, 2012; Lasiuk, Comeau, \& Newburn-Cook, 2013; Maheu \& Thorne, 2008; Muscat, 
2010; Thorne et al., 2010) called interpretive description. Interpretive description was developed because of the limitations of traditional qualitative research methodologies (Thorne, Kirkham, \& MacDonald-Emes, 1997) and is designed to be "more responsive to the experience-based questions" (Thorne, Kirkham, \& O'Flynn-Magee, 2004, p. 1). Interpretive description presumes some theoretical knowledge is brought to the research, which can then be used to provide an initial model around which design and analytical decisions can be made (Thorne et al., 2004). However, interpretive description does not define a series of specific steps that will automatically lead to the discovery of new knowledge. Instead, it draws on the theoretical foundations of ethnography, grounded theory and phenomenology and uses them as a conceptual model rather than from a purist perspective (Thorne, 2008). Because of these qualitative roots, interpretive description can also draw on a broad range of research methods. The freedom of not being constrained by the limitations of more traditional qualitative research methodologies and the ability to develop an initial model using the current literature is consistent with the requirements of this study. 


\section{Method}

As described in the methodology section, this research is focused on understanding how people's attitudes are defined by their experiences. In order to achieve this, Personal construct theory (PCT) was adopted. PCT was first proposed by Kelly (1955) to explain how people conceptualise their experiences. It views individuals as scientists, who actively try to understand the world around them by attempting to recognise repeating patterns in their experiences (Jankowicz, 2005), and then representing those patterns as bi polar concepts called constructs (Paszkowska-Rogacz \& Kabzinska, 2012).

The repertory grid (repgrid) interview method, also developed by Kelly, is a way to tap into people's constructs. It is being increasingly used in information systems research because it helps to elicit participants underlying attitudes and beliefs (Bernard \& Flitman, 2002; Hunter \& Beck, 2000; Tan \& Hunter, 2002; Whyte \& Bytheway, 1996), and can be used for both quantitative and qualitative research (Marsden \& Littler, 2000). Because repgrids allow access to peoples' underlying attitudes and beliefs it is an ideal tool to help determine how those underlying factors, and people's experiences of those factors, form their attitudes to reuse, refurbishing and recycling (Figure 1).

A repgrid consists of a matrix of elements and constructs, the elements are areas of interest whilst the constructs are descriptions that are used to explain the elements, and are created as poles. The elements and constructs can be created either by the interviewer (supplied element) or the subject (elicited element) (Tan \& Hunter, 2002). For this research, the elements were developed by the researcher, with all but one of the constructs being elicited from the participants, which is consistent with the approach suggest by Jankowicz (2005). This single supplied construct called an overall summative construct was supplied so that Honey's content analysis could be conducted. A description of Honey's content analysis is provided in section 4.8. Whilst the use of preconceived elements does have the potential to introduce some researcher bias, it is the preferred approach when trying to compare participants' mental models as it acts as a common denominator (Wright, 2006). The elements themselves are aligned between the opposing poles of the constructs and each element is rated using either a Likert or dichotomous yes/no scale to indicate which pole it is closer to. A five point Likert scale was adopted for use in this study, because "To use a wider range is probably spurious, since you'd be asking people to make finer discriminations than they can 
accurately express in a consistent way across the whole grid." (Jankowicz, 2005, p. 36). Because elements were supplied and not elicited', a process of element familiarization (Fallman \& Waterworth, 2005) was undertaken with each participant prior to the interview, to ensure they fully understood the context of each element.

In total five elements were used during the interview process. These consisted of three elements relating to reusing, refurbishing, and recycling old mobile phones as these represented the area of interest. Two grounding elements were also provided, "the worst thing to do with an old mobile phone" and "the ideal thing to do with an old mobile phone" to determine what the participant felt were the worst and ideal things to do. Whilst the two grounding elements may not seem as specific as the elements relating to reusing, refurbishing, and recycling, they are provided in order to try to understand the area under investigation from the participants' perspective. This means not forcing participants to pick reuse, refurbish or recycling if those elements do not exactly fit their world perspective. For instance, from the participants' perspective, the ideal thing to do may consist of areas from both reuse and recycling whilst the same concept also applies to the worst thing to do. This approach is supported in that it has been proposed "There are no hard and fast rules regarding element set selection." and "The essential issue must be that the person is able to construe the set of elements and finds them relevant.” (Pope \& Denicolo, 1993, p.152).

A triadic elicitation process was used during the interviews, where participants were presented with a group of three elements, and asked which two elements were similar to each other but were different from the third. An example, is where participants were asked, of these three areas, "Reusing an old mobile phone", "Purchasing a refurbished mobile phone" and the "Ideal thing to do with old mobile phone", which two were similar but different from the third. The phrase or word the participant used to described why two of the elements were similar was captured as an initial construct at the emergent pole of the repertory grid. For example, "Reusing an old mobile phone" and "Purchasing a refurbished old mobile phone" could be similar because of the idea of "reusing parts for self'. The phrase or word that the participant then used to describe why the third element was not similar was then captured as an initial construct at the implicit pole of repertory grid. In this example, the "Ideal thing to do with old mobile phone" might be not similar because of the idea that it's "still in one piece" (Figure 2). 


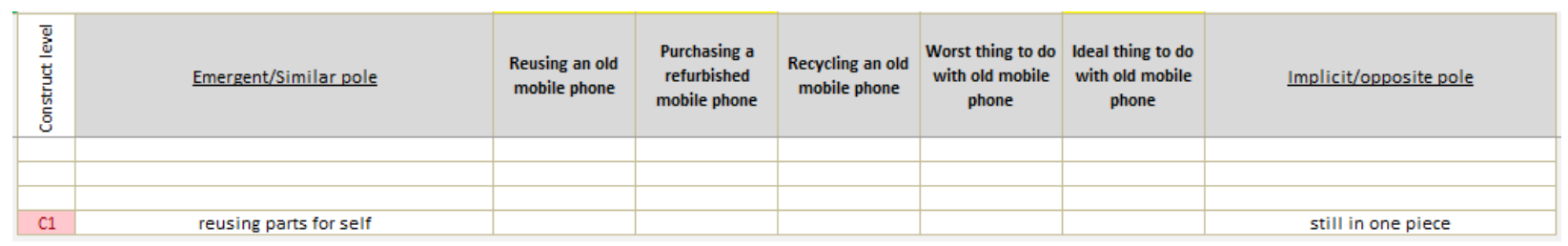

Figure 2. Example construct capture

In an attempt to access the participants core constructs, a laddering interview technique was also adopted.

\subsection{Laddering}

Laddering is an interview technique that was suggested by Hinkle (1965), which is also based on the notion of personal constructs found in PCT. Hinkle (1965, p. 14) describes it as "The hierarchical technique for eliciting the superordinate constructs of the preferred self-hierarchy". It is achieved by asking a series of "why" or "what" questions to ladder up or down when eliciting different level constructs. This continues for each construct until no new ideas or descriptions emerge. For this research, participants were laddered upwards (Figure 3) in an attempt to access their core constructs, because "Core constructs' remind the person of who s/he is; what really, really matters to them and what they value in existence....." (Jankowicz, 2005, p. 83). 


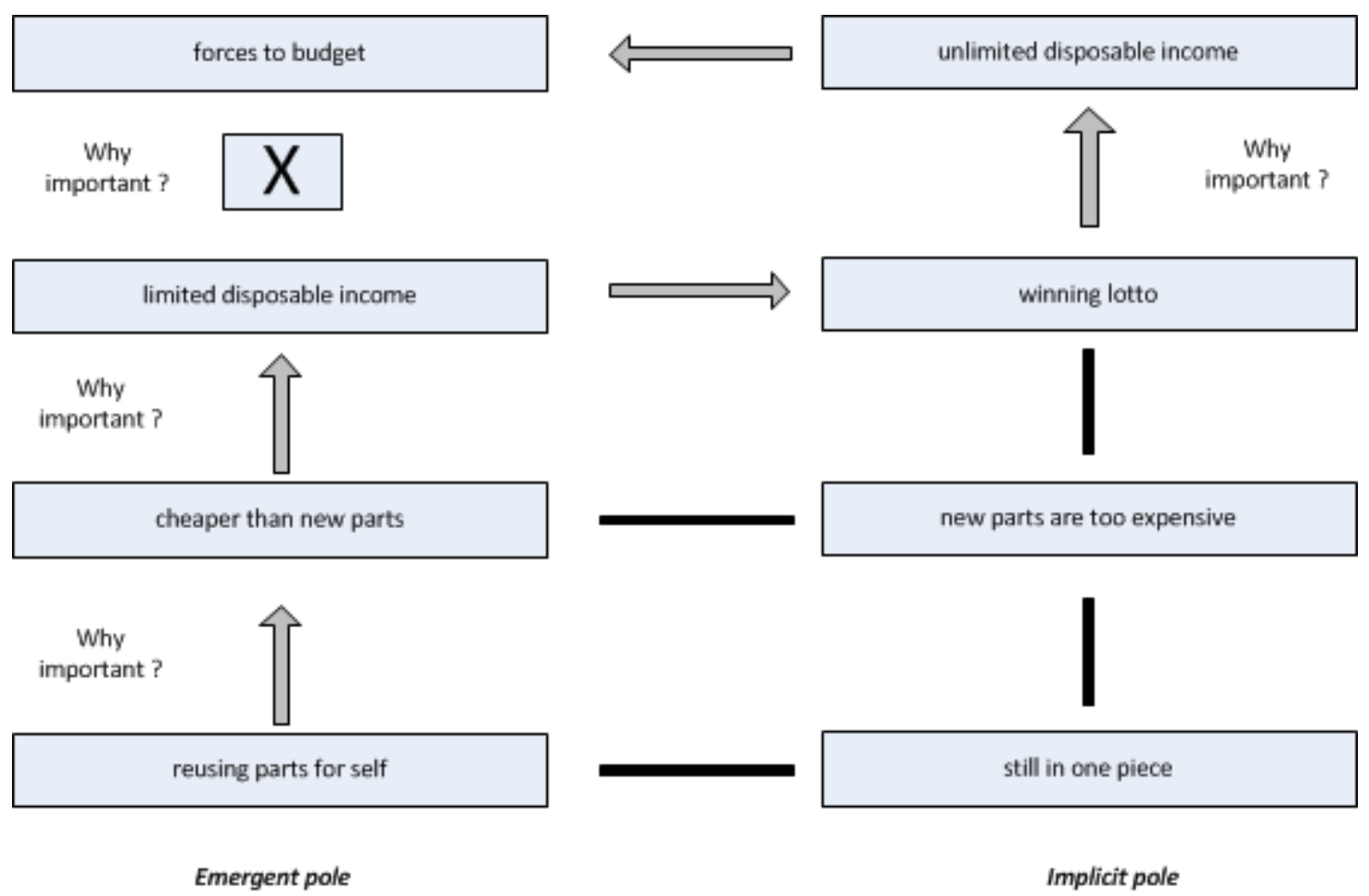

Figure 3. Example construct ladder

As part of this process, participants were asked to explain in one or a few words why they felt the initial construct they had provided at the emergent pole, during the triadic elicitation (Figure 2) was important to them; this was then captured as a higher-level construct on the construct ladder (Figure 3). They were then asked to provide a contrasting statement in an attempt to capture the opposing construct; the response was then captured at the implicit pole. This process continued until the participant felt they could no longer answer the why question, or that repeating answers were beginning to emerge. In some instances where a participant was unable to describe why a construct was important to them, the interview crossed over to the opposing pole where the "why" question was repeated (Figure 3). If the pole of a new construct emerged, the participant was then asked for a contrasting statement and the interview moved back to the original side of the ladder. Once the core construct had been reached at the top of a construct ladder, participants were then asked to first rate the three elements that were used to elicit the original subordinate construct on a scale of one to five with one being closer to the emergent pole and five being closer to the implicit pole (Figure 4). This process continued until all of the possible triadic combinations of elements were exhausted. During the catchall phase, at the end of the interview, participants were asked to consider if there were any areas they felt had not already been covered during the construct elicitation part of the interview but 
were relevant to the area under investigation. As part of this process, the participants were reminded of the concepts behind the elements. Any constructs established during the catch all phase were also subject to the same laddering approach used during the triadic elicitation.

\begin{tabular}{|c|c|c|c|c|c|c|c|}
\hline 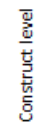 & Emergent/Similar pole & $\begin{array}{c}\text { Reusing an old } \\
\text { mobile phone }\end{array}$ & $\begin{array}{c}\text { Purchasing a } \\
\text { refurbished } \\
\text { mobile phone }\end{array}$ & $\begin{array}{l}\text { Recycling an old } \\
\text { mobile phone }\end{array}$ & $\begin{array}{c}\text { Worst thing to do } \\
\text { with old mobile } \\
\text { phone }\end{array}$ & $\begin{array}{l}\text { Ideal thing to do } \\
\text { with old mobile } \\
\text { phone }\end{array}$ & Implicit/opposite pole \\
\hline sc1.3 & forces to budget & 3 & 5 & 4 & 1 & 5 & unlimited disposable income \\
\hline sc1.2 & limited disposable income & & & & & & winning lotto \\
\hline sc1.1 & cheaper than new parts & & & & & & new parts are too expensive \\
\hline $\mathrm{C} 1$ & reusing parts for self & & & & & & still in one piece \\
\hline
\end{tabular}

Figure 4. Example laddering result

Participants were then presented with the overall summative construct. Whilst they were not asked to ladder up from this construct, as it needed to be kept the same for all participants, they were asked to rate it in the same way the core constructs were rated (Figure 5). The aim of this was to provide a common construct that could be used to compare all the other constructs across the group using Honey's content analysis.

\begin{tabular}{|c|c|c|c|c|c|c|c|}
\hline 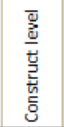 & Emergent/Similar pole & $\begin{array}{c}\text { Reusing an old } \\
\text { mobile phone }\end{array}$ & $\begin{array}{l}\text { Purchasing a } \\
\text { refurbished } \\
\text { mobile phone }\end{array}$ & $\begin{array}{l}\text { Recycling an old } \\
\text { mobile phone }\end{array}$ & $\begin{array}{l}\text { Worst thing to do } \\
\text { with old mobile } \\
\text { phone }\end{array}$ & $\begin{array}{c}\text { Ideal thing to do } \\
\text { with old mobile } \\
\text { phone }\end{array}$ & Implicit/opposite pole \\
\hline sup & Overall - Most effective & 1 & 4 & 3 & 5 & 2 & Overall - Least effective \\
\hline sc10.2 & enjoy repurposing existing components & 1 & 3 & 4 & 5 & 2 & dislike repurposing components \\
\hline sc10.3 & build something different & & & & & & build something the same \\
\hline sc10.2 & can use components & & & & & & can't use component \\
\hline sc10.1 & does not allow for disassembly & & & & & & allows to be disassembled \\
\hline $\mathrm{c} 10$ & no longer have the unit & & & & & & you have control of the unit \\
\hline sc9.2 & keep in touch with people & 3 & 1 & 5 & 4 & 2 & lose touch with people \\
\hline sc9.1 & so you can make phone calls & & & & & & unable to make phone calls \\
\hline C9 & because you have possession of a mobile phone & & & & & & you don't have a mobile phone \\
\hline$s c 8.2$ & you can no longer enjoy dismantling it & 5 & 3 & 2 & 1 & 4 & you can enjoy dismantling \\
\hline sc8.1 & you are unable to alter unit & & & & & & you are able $o$ alter the unit \\
\hline C8 & you no longer have the unit & & & & & & you don't lose control of the unit \\
\hline sc7.5 & I love it & 1 & 2 & 4 & 5 & 3 & I hate it \\
\hline sc7.4 & I like to know things & & & & & & ignorance \\
\hline sc7.3 & learn new things & & & & & & don't learn new things \\
\hline sc7.2 & I find it a challenge & & & & & & I find it easy \\
\hline sc7.1 & because I enjoy it & & & & & & I find it un-enjoyable \\
\hline c7 & because you can dismantle both & & & & & & no longer have unit \\
\hline erfit & I Ann't like th he etresead & ? & 1 & $A$ & 5 & 2 & I like to he etresear \\
\hline
\end{tabular}

Figure 5. Example construct ladder with supplied construct - SUP

Due to the laddering process and catchall phase, interviews took between one and two hours with the average length being approximately one hour and 25 minutes. All interviews were audio recorded and supplemented with the creation of interview memos. 


\subsection{Sample size and selection}

Tan and Hunter (2002) suggest a sample size between 15 and 25 is large enough to generate enough constructs within the repgrid technique, however, Winter (2003) suggests a smaller sample size of between 10-17 is sufficient to elicit a satisfactory number of factors, whilst other studies have only used 2 participants for repgrid generation (Phythian \& King, 1992). For this research, 12 participants were interviewed using a convenience sampling approach with each interview expected to produce at least 10 elicited constructs and a single supplied construct. These 11 constructs, did not include additional constructs identified during the catch all phase towards the end of each interview. This minimum number of expected constructs fell with the range of 10 to 15 constructs suggested by Jankowicz (2005).

Whilst a convenience sampling approach was adopted, care was taken to ensure participants were as diverse as possible. However, none of the participants appeared to hold extreme passionate views on either the environment or environmental issues. Two participants did mention they were either vegetarian or vegan but this was more focused on their concern for animal welfare rather than a greater concern for the environment in general. The results of their interviews did not vary significantly from other participants in terms of perceived levels of environmental concern. It should also be noted, that all participants were from the wellington region, which has the highest mean household income level in the country, as well as the highest number of high-skilled occupations compared to other territorial authorities (Ministry of Business Innovation \& Employment, 2015).

\subsection{Word (content) cloud}

McNaught and Lam (2010) describe a word cloud as a way to represent graphically, the frequency in which words appear in a piece of text and are good for preliminary or validation analysis. Words that are used more frequently in the piece of text occupy a more prominent position in the word cloud. The idea, is that words that are expressed more frequently are likely to indicate areas of greater importance. It has been described as applicable method in qualitative content analysis as "any material that can be studied using content analysis can therefore be visualized through the generation of a content cloud" (Cidell, 2010, p. 514). For this research, word clouds (Figure 6) were created using NVIVO. For individuals, all of the elicited constructs were used to generate the word clouds, however, for the group analysis all of the participants elicited constructs were combined to 
produce an overall group word cloud (Appendix 3). The resultant word clouds helped determine the strength of the themes that appeared to emerge from the repgrids during construct analysis.

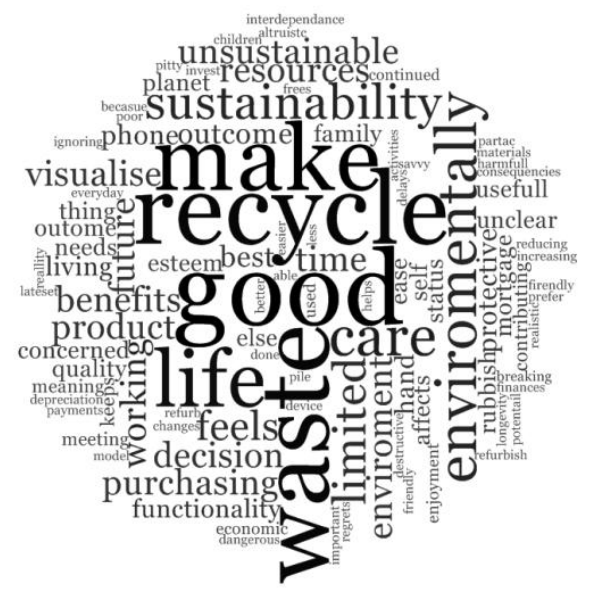

Figure 6. Example word cloud

\subsection{Visual focusing}

Visual focusing is a repgrid analysis technique, that is used to help reveal if there are any potential relationships between the constructs and elements within a repgrid (Tan \& Hunter, 2002). The process requires the realignment of the repgrid so similarly rated constructs and elements are aligned which can result in construct poles being reversed. This process results in the emergence of highlevel patterns of data from within the repgrid (Edwards, McDonald, \& Young, 2009). For this research, the process of visual focusing was used during initial analysis to highlight the potential data patterns and relationships, and was enhanced by using conditional formatting within an excel spreadsheet that highlighted all ratings below 3 as blue, all ratings above 3 as yellow and all 3's as grey (Figure 7).

\begin{tabular}{|c|c|c|c|c|c|c|c|}
\hline & & & & & & & \\
\hline & & E1 & E2 & E3 & E4 & E5 & \\
\hline & & $\begin{array}{l}\text { Re using an old } \\
\text { mobile phone }\end{array}$ & $\begin{array}{l}\text { Purchasing a } \\
\text { refurbished } \\
\text { mobile phone }\end{array}$ & $\begin{array}{l}\text { Recycling an } \\
\text { old mobile } \\
\text { phone }\end{array}$ & $\begin{array}{l}\text { Worst thing to } \\
\text { do with old } \\
\text { mobile phone }\end{array}$ & $\begin{array}{l}\text { Ideal thing to } \\
\text { do with old } \\
\text { mobile phone }\end{array}$ & \\
\hline SUP & Overall - Most effective & 1 & 1 & 2 & 5 & 2 & Overall - Least effective \\
\hline sc13.2 & not waste of time & 1 & 1 & 3 & 3 & 2 & ease of use \\
\hline sc12.4 & saves time & 1 & 1 & 4 & 4 & 2 & give up \\
\hline C11 & Usefull (limited) & 1 & 1 & 1 & 2 & 3 & Not usefull \\
\hline C10 & being used again & 1 & 1 & 3 & 1 & 4 & recycle \\
\hline C9 & best use of product & 1 & 1 & 2 & 3 & 3 & reuse \\
\hline C8 & best use of product & 1 & 1 & 1 & 4 & 4 & recycle \\
\hline sc7.1 & enviromentally sound & 1 & 1 & 1 & 5 & 2 & waste \\
\hline sc6.1 & creates less rubbish & 1 & 1 & 1 & 4 & 1 & waste of technology \\
\hline sc5.1 & recycle & 1 & 2 & 1 & 3 & 1 & rubbish pile up \\
\hline sc4.4 & quality of life & 1 & 1 & 2 & 3 & 2 & restriction on wellbeing \\
\hline sc3.4 & quality of everyday living & 1 & 1 & 3 & 1 & 5 & healthy enviroment (recycling) \\
\hline sc2.3 & enviromental concerns & 1 & 1 & 2 & 5 & 1 & toxic waste \\
\hline sc1.6 & family is the most important thing we have & 2 & 3 & 1 & 3 & 2 & no regrets for living life \\
\hline
\end{tabular}


Figure 7. Visual focusing repgrids

Following visual focusing both the sum of differences and the percentage similarity were also calculated for both the elements and the constructs. This was done because in qualitative content analysis, it is possible to use quasi-statistical analysis methods (Neergaard, Olesen, Andersen, \& Sondergaard, 2009) that can help describe patterns in the data (Sandelowski, 2000).

\subsection{Sum of differences}

The sum of differences is a mathematical calculation designed to highlight the differences between the ratings for both elements and constructs, with the aim of trying to find the smallest difference (Jankowicz, 2005). It is useful for analysing the difference between both the elements and constructs, and is a non-statistical way to present the data that is reflected in the visual analysis. It is useful when examining individual repgrids but is limited when wanting to compare repgrids from different participants. However, in order to compare across repgrids the sum of differences needs to be converted into a percentage similarity.

\subsection{Percentage similarity}

The percentage similarity is calculated using the sum of differences. It provides a consistent measurement that can be used to compare repgrids across a group to measure similarity within a single repgrid (Jankowicz, 2005). As with the sum of differences, it can be calculated for both elements and constructs. For elements it is possible to calculate the percentage similarity within a repgrid and when the same elements are used across several repgrids it allows for a comparison across repgrids. For constructs, the percentage similarity only allows for comparison within a repgrid, however, the result of construct reversals also needs to be captured to identify what affects these have on the percentage similarity. To allow for the comparison of constructs across repgrids the percentage similarity needs to be used in conjunction with a summative construct and Honey's content analysis.

For this research, the percentage similarity was captured in an element rating matrix (Figure 8) and construct rating matrix (Figure 9). 


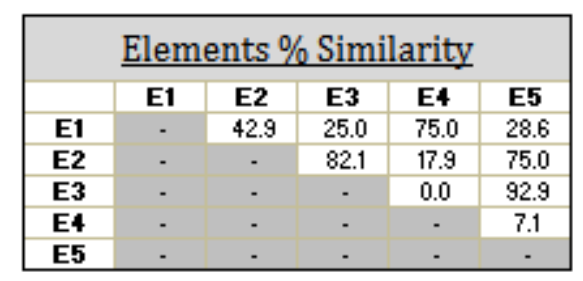

Figure 8. Elements percentage similarity matrix

\begin{tabular}{|c|c|c|c|c|c|c|c|c|c|c|c|c|c|c|c|c|c|}
\hline \multicolumn{18}{|c|}{ Constructs \% Similarity } \\
\hline \multirow{16}{*}{ 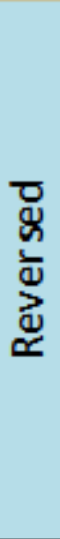 } & \multirow{3}{*}{$\begin{array}{c}\text { Honeys } \\
\text { CA }\end{array}$} & & \multicolumn{15}{|c|}{ Un reversed } \\
\hline & & & & SUP & sc13.3 & $\operatorname{se12.3}$ & sc11.2 & $\operatorname{se10.1}$ & $\operatorname{se9.1}$ & $5 \mathrm{c8} .2$ & sc7.1 & sc6.1 & $\operatorname{se-5.2}$ & sct.1 & C3 & $\sec 2.2$ & sc1.2 \\
\hline & & & SUP I & $\cdot$ & -40 & -80 & 70 & .70 & 60 & 70 & 90 & -90 & 80 & 70 & 80 & 60 & 60 \\
\hline & L & $\mathrm{R}$ & sc13.3 & 60 & - & 40 & -30 & 30 & -20 & .70 & -50 & 50 & -60 & .70 & -40 & -60 & -60 \\
\hline & I & $\mathrm{R}$ & sc12.3 & 80 & 40 & . & .70 & 90 & -60 & .70 & -90 & 90 & -80 & .70 & -60 & -60 & -60 \\
\hline & 1 & & sc11.2 & 70 & 30 & 90 & $\cdot$ & -60 & 90 & 60 & 80 & -80 & 70 & 60 & 50 & 70 & 70 \\
\hline & I & $\mathrm{R}$ & $\operatorname{se10.1}$ & 70 & 30 & 90 & 100 & . & .70 & -60 & -80 & 80 & .70 & -60 & -50 & -50 & .50 \\
\hline & L & & $\operatorname{se9.1}$ & 60 & 20 & 80 & 90 & 90 & $\cdot$ & 50 & 70 & .70 & 60 & 50 & 40 & 60 & 60 \\
\hline & I & & $\operatorname{ses} 8.2$ & 70 & 70 & 70 & 60 & 60 & 50 & . & 80 & -80 & 90 & 100 & 70 & 90 & 90 \\
\hline & $\mathrm{H}$ & & sc7.1 & 90 & 50 & 90 & 80 & 80 & 70 & 80 & - & -100 & 90 & 80 & 70 & 70 & 70 \\
\hline & $\mathrm{H}$ & $\mathrm{R}$ & se6.1 & 90 & 50 & 90 & 80 & 80 & 70 & 80 & 100 & - & -90 & -80 & .70 & .70 & .70 \\
\hline & 1 & & $\operatorname{se} 5.2$ & 80 & 60 & 80 & 70 & 70 & 60 & 90 & 90 & 90 & - & 90 & 80 & 80 & 80 \\
\hline & 1 & & sct.1 & 70 & 70 & 70 & 60 & 60 & 50 & 100 & 80 & 80 & 90 & - & 70 & 90 & 90 \\
\hline & 1 & & C3 & 80 & 60 & 60 & 50 & 50 & 40 & 70 & 70 & 70 & 80 & 70 & . & 60 & 60 \\
\hline & L & & $\operatorname{se} 2.2$ & 60 & 60 & 80 & 70 & 70 & 60 & 90 & 70 & 70 & 80 & 90 & 60 & - & 100 \\
\hline & L & & sc1.2 & 60 & 60 & 80 & 70 & 70 & 60 & 90 & 70 & 70 & 80 & 90 & 60 & 100 & . \\
\hline
\end{tabular}

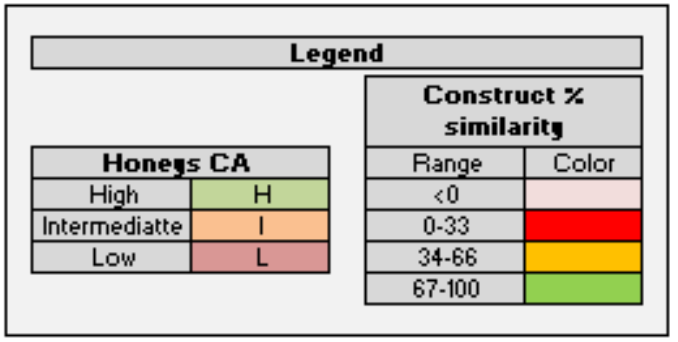

Figure 9. Construct percentage similarity matrix

After the visual analysis, individual grid elements were reviewed for their percentage similarity. This included reviewing how the reuse, refurbish and recycle elements compared to the grounding elements "worst" and "ideal". Constructs were also then reviewed against each other by working through each construct and reversing it to ensure the highest degree of similarity possible between constructs was achieved. Reversing a construct is where the words at the pole of the construct are reversed and all the construct ratings are inverted. This is possible because a reversed construct has the same meaning as the un-reversed construct (Jankowicz, 2005). Where a higher degree of similarity was achieved because of the reversing of a construct, this was noted. For the individual repgrids comparisons, all constructs were tested against each other in order to get the highest 
percentage similarity. When constructs were tested for use in Honey's content analysis, all constructs within a grid were only tested against the overall supplied construct.

\subsection{Content analysis}

The conventional approach to content analysis is used when there is limited theory or research in an area. It avoids the use of preconceived categories, instead the researcher uses an inductive approach to data analysis, which allows categories to emerge (Hsieh \& Shannon, 2005) as a result the researcher, becomes grounded in the data (Kondracki, Wellman, \& Amundson, 2002). In directed content analysis, the intent is to test or extend a theoretical model and it follows a more structured approach (Hsieh \& Shannon, 2005). It is described as being deductive in nature and results in the researcher establishing some predetermined key words or categories, which have been established using sources such as the current literature (Kondracki et al., 2002). This approach does not limit the ability of new knowledge to emerge, as data that is unable to be coded against the predetermined categories, is re-examined later to determine if it represents a new category (Hsieh \& Shannon, 2005). It is also possible to apply both approaches, as they are not deemed to be mutually exclusive (Kondracki et al., 2002). Deductive content analysis is appropriate where there is some pre-existing knowledge. Because a pool of theoretical knowledge already existed in the area that was being examined this allowed for the development of priori categories, subcategories and a theoretical model, therefore a deductive content analysis approach was adopted.

When it comes to content analysis, researchers also need to determine whether you wish to look at manifest content, latent content, or both. Kondracki et al. (2002) described these various types of content. Manifest content is that which is easily observable on the surface of the data. It tends to emerge when coding the data and examines the frequency of which words occur. However, latent content is more difficult to identify, and requires a more complex process. It requires the researcher to attempt to identify deeper meanings from the data other than that which is seen on the surface. Whilst this research focuses mainly on manifest content, some latent content analysis was attempted during repgrid analysis.

When coding during content analysis it is important to understand that "...a word or a phrase does not contain its meaning as a bucket contains water, but has the meaning it does by being a choice about its significance in a given context." (Bliss, Monk \& Ogborn 1983: cited in Miles \& Huberman, 
1984). Using this research as an example, whilst the constructs form the meaning units for the content analysis (Jankowicz, 2005), the context in which the constructs were created should also be considered. Therefore, in order to determine where a core construct belonged in the coding matrix a number of areas were reviewed. These included:

- An examination of the underlying subordinate constructs that generated the core constructs.

- The repgrid elements that led to the original construct elicitation.

- The background demographic questions captured using the short questionnaire at the start of the interview.

- An interview memo.

- The interview recording.

In order to ensure no potential new categories or subcategories were missed, any areas that did not appear to fit into the original coding matrix were re-examined and new categories and sub categories created where required (Hsieh \& Shannon, 2005). In order to provide validity in the coding process a selection of sample codes along with supporting information and documentation were provided to the research supervisor to review to determine if both the researcher and the supervisor were able to identify similar coding selections.

\subsection{Honey's content analysis}

In order to ensure that content analysis captures some of the value presented in the ratings matrix, Honey's content analysis was used. It is a similar process to other forms of content analysis, however, as Jankowicz (2005) describes, the approach of Honey's content analysis allows for the inclusion of information that reflects the ratings data which otherwise would be lost using more conventional content analysis approaches. This is achieved by asking participants to rate all elements against a researcher defined construct in the same way elicited constructs were rated. For this research the construct that was provided was "Overall-Most effective $<>$ Overall-Least effective". It can be described as a summative or overall construct that is designed to capture how participants feel overall about the area being explored. By getting all participants to rate elements along the same overall construct, a point of construct similarity is created. It is then possible to determine how all participant provided constructs rate against this overall construct using a high, medium, or low (H-IL) indices comparison that is created from the percentage similarity rating (Appendix 2). 


\subsection{Summary of methods}

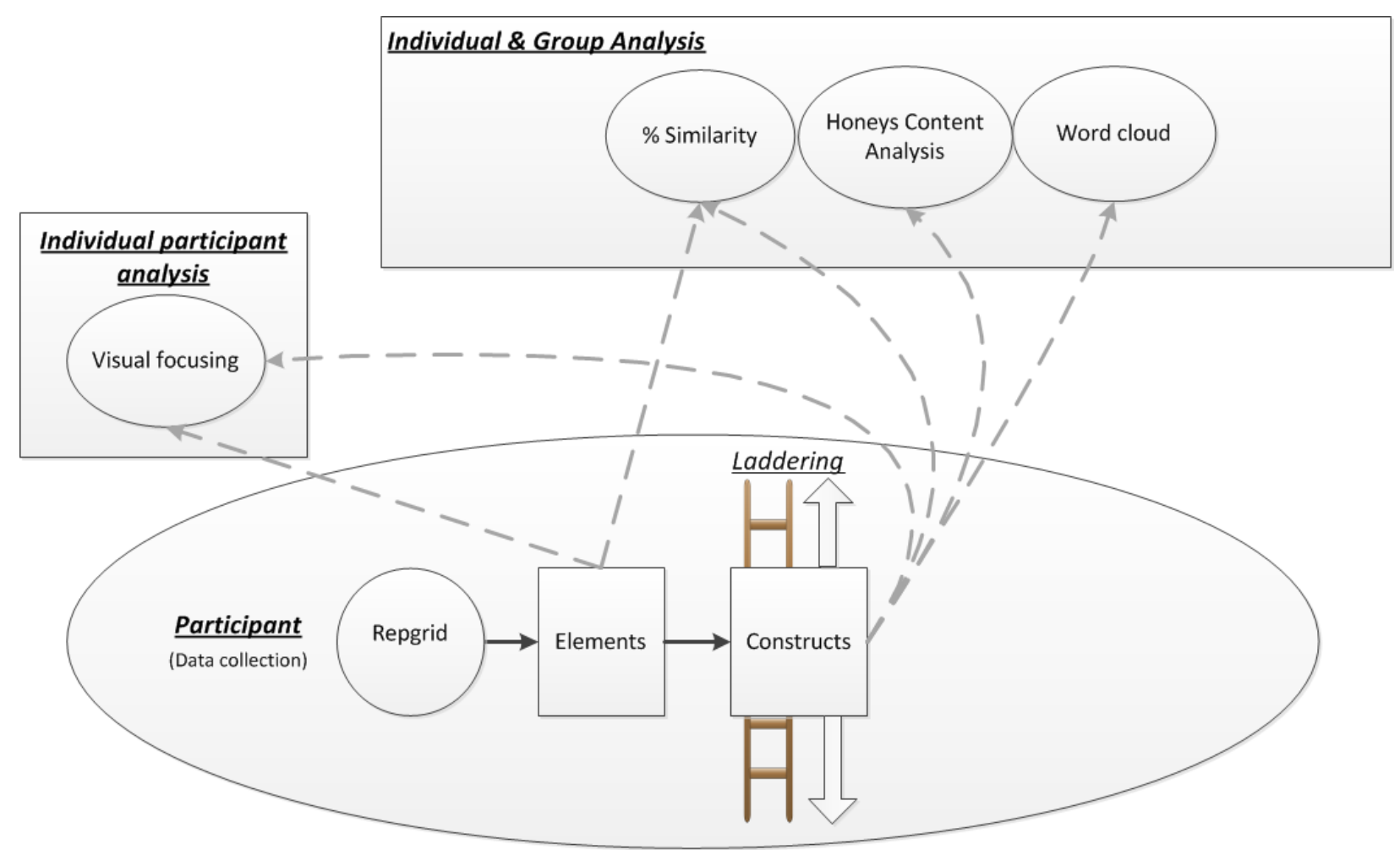

Figure 10. Summary of methods

Figure 10 summarises how all the methods presented in this research work together. During data collection, each participant helps develop a repgrid that produces a set of rated elements and constructs. If laddering is used, constructs may also be laddered up or down depending on the purpose of the research. For this research laddering up was utilised. When it comes to analysis, visual focusing is used to analyse individual participants' repgrids, whilst the percentage similarity, Honey's content analysis and word clouds are used for both individual and group analysis. However, whilst visual focusing and the percentage similarity provide the ability to analyse both elements and constructs, Honey's content analysis and word clouds were only used to analyse constructs.

It also important to identify that while both content analysis and Honey's content analysis are presented in the method section, only Honey's content analysis is presented in the summary. This is because Honey's content analysis as an extension of content analysis. The sum of differences is also not presented in the summary though it is described in the method. This is because the sum of differences is required to calculate the percentage similarity, but is not used in the analysis. 


\subsection{Initial testing}

Initial repgrid testing was done using Idiogrid elicitation software, however, this limited the ability to undertake laddering when attempting to identify core constructs during the elicitation process. It also did not allow for the capture of constructs during the catch all phase of the interview. Since these were two important aspects of the research it was decided to not use the software but instead use Microsoft Excel. A workbook was created that could be used to capture all constructs during the elicitation and laddering process. Participants had their own version of the workbook template. The use of excel allowed for the easy visual manipulation of both the elements and constructs as well as the easily recalculation of any mathematical formula.

\subsection{Ethics}

Ethics approval is an essential aspect of any research that involves human participants as it ensures their rights are protected and, the risk of any potential harm is minimized. Ethics approval for this research was sought from, and approved by, the School of Information Management Human Ethics Committee. As part of receiving ethics approval, participants were provided with an information sheet (Appendix4) outlining the importance of the research and the role they would play. They were also provided with a list of background demographic questions (Appendix 4b) and asked to sign an informed consent form (Appendix 4c) to acknowledge they understood the implications of participating in the research. 


\section{Results}

The results chapter is comprised of three sections. The first section, reviews the results of each participant in terms of both the elements in the individual repertory grid and the general results suggested by their constructs. The second section reviews the repertory grids as a group and the third, the results of the content analysis. Whilst the results section is primarily about delivering the research results, "It is common practice to include some discussion within the results section of qualitative research and follow with a concluding discussion.”(Anderson, 2010, p. 5). Therefore, some discussion is also included in the results section. As previously mentioned, for the purposes of this study some terms have the same meaning, for instance, subcategory and factor (Please refer to table 1).

\subsection{Individual participant analysis}

This section will briefly describe each of the participants' demographic information, and provide a summary of their repertory grid. Five elements were present to each participant, three reflected the area under investigation which were reuse, refurbish and recycle, whilst two were grounding elements, the worst thing to do and ideal thing to do.

\section{Participant 1}

Participant 1 is aged between 18 and 34 with an income range of $\$ 50,000$ to $\$ 75,000$. They are employed as a full time support leader and their highest academic achievement was a high school qualification. Analysis of the percentage similarity across elements identified that the recycle element had an 88.6 percent similarity to the ideal element, whilst both the reuse and refurbish elements had a 50 percent and 52.3 percent similarity respectively. Reuse had an 88.6 percent similarity to the refurbish element and was the highest of the non-grounding elements, whilst reuse only a 52.3 percent similarity to the recycle element which was the lowest of the non-grounding elements. This suggests the participant views recycling as the most ideal thing to do with a used mobile phone and that reuse and refurbishing were seen as the most similar. During analysis of the repgrid constructs, three of the elicited constructs needed reversing to achieve the highest possible percentage similarity across constructs. Two constructs were more closely associated with the overall construct, with a 90 percent similarity, and were related the subcategories environmental awareness and social norm/culture. They suggested the participant is concerned about their lack of thought when it 
comes to environmental issues and fears how any negative environmental consequences could directly them.

\section{Participant 2}

Participant 2 is aged between 35 and 55 with an income range of $\$ 25,000$ to $\$ 50,000$. They are part time self-employed as a midwife in the health sector and their highest academic achievement was at the postgraduate level. Analysis of the percentage similarity between elements indicated that recycle had a percentage similarity of 87.5 percent to the ideal element. This was a significantly higher alignment with the ideal element compared to both the reuse and refurbish elements at 69.6 percent and 62.5 percent respectively. The percentage similarity between reuse and recycle was 82.1 percent, which was the highest of the non-grounding elements, whilst the percentage similarity between refurbish and recycle was 71.4 percent, which was the lowest of the non-grounding elements. This suggests the participant views reuse, refurbishing and recycling, all as positive, however, recycling is seen as the more ideal way to manage used mobile phones, whilst reuse and recycling are seen as the most similar. During analysis of the repgrid constructs, two of the elicited constructs needed to be reversed in order to achieve the highest possible percentage similarity across constructs. Five constructs were more closely associated with the overall construct, all having the same percentage similarity of 80 percent. These constructs were related to the subcategories age of technology, residual value, social norm/culture, and environmental awareness. They indicated concern for the environment, needing to be well informed with money, the lack of need for the latest technology and the need to be a role model.

\section{Participant 3}

Participant 3 is aged between 18 and 34 with an income range of $\$ 50,000$ to $\$ 75,000$. They are employed full time as an administrator and their highest academic achievement was at the undergraduate level. Analysis of the percentage similarity between elements identified that both reuse and refurbish had identical percentage similarity to the ideal element at 63.6 percent whilst recycle had the highest percentage similarity at 75 percent. Both reuse and refurbish had the highest percentage similarity of the non-grounding constructs at 100 percent whilst both reuse and refurbish had and 88.6 percent similarity to recycle, which was the lowest of the non-grounding elements. This indicates the participant views recycling as more ideal than reusing or refurbishing but does not differentiate between reusing and refurbishing mobile phones. Five constructs were more closely 
associated with the overall construct all with a percentage similarity of 80 percent. These constructs were associated with the subcategories ease, functioning, perception, and environmental awareness. They indicated that this participant felt being environmentally friendly and avoiding waste was important especially when phones are still functioning, however, the outcome of recycling is not always clear.

\section{Participant 4}

Participant 4 is aged between 74 and 91 with an income range of $\$ 50,000$ to $\$ 75,000$. They are retired and their highest academic achievement was a high school qualification. Analysis of the percentage similarity across elements identified recycle as having the highest percentage similarity to the ideal element at 73.2 percent, followed by reuse at 66.1 percent and refurbish at 62.5 percent. There was a 96.4 percent similarity between reuse and refurbish, which was the highest of the nongrounding elements, whilst refurbish had a 71.4 percent similarity to recycle which was the lowest of the non-grounding elements. This, as with previous participants, indicates that recycling is perceived as the better approach to managing a used mobile phone whilst reusing and refurbishing mobile phones are seen as highly similar. Two constructs had a 90 percent similarity to the overall construct and were associated with the subcategory environmental awareness and related to the idea of managing waste along with concern for the environment.

\section{Participant 5}

Participant 5 is aged between 35 and 55 with an income range of $\$ 50,000$ to $\$ 75,000$. They are employed part time as a nurse in the health sector and their highest academic achievement was at the undergraduate level. Analysis of the percentage similarity between elements indicated recycle had a 92.9 percent similarity to the ideal element; this was followed by refurbish at 75 percent and reuse at 28.6 percent. Refurbish had an 82.1 percent similarity to recycle and was the highest of the nongrounding elements whilst reuse only had a 25 percent similarity to recycle, which was the lowest of the non-grounding elements. This suggests that this participant strongly feels that recycling is the ideal thing to do with a used mobile phone and that recycling is seen more similar to refurbishing than reusing. Two constructs had the highest percentage similarity at 90 percent to the overall construct and were related to the subcategories of environmental awareness and social norm/culture. These constructs reflected both concern for the environment and the idea that somebody else should benefit from used mobile phones. 


\section{Participant 6}

Participant 6 is aged between 35 and 55 with an income range greater than $\$ 100,000$. They are employed part time as a soldier in the Zealand Defense Force (NZDF) and their highest academic achievement was a trade certificate. Analysis of the percentage similarity across elements identified that reuse had a high 81.8 percent similarity to the ideal element, followed by recycle at 72.7 percent and refurbish 54.5 percent. Reuse and recycle had a 72.7 percent similarity to each other and was the highest of the non-grounding elements, whilst refurbish had a 50 percent similarity to recycle, which was the lowest of the non-grounding elements. This suggests that the participant views reuse as closer to the ideal thing to do whilst reusing and recycling were the most closely associated. Only one construct was closely associated with the overall construct with a 100 percent similarity. This construct was related to the subcategory functioning and reflected that the participant felt that the repairing of a mobile phone was the area most associated with a used mobile phone whilst concern for the environment only had an 80 percent similarity to the overall construct.

\section{Participant 7}

Participant 7 is aged between 35 and 55 with an income range of $\$ 50,000$ to $\$ 75,000$. They are employed full time as a fitter and turner and their highest academic achievement was a trade certificate. Analysis of the percentage similarity between elements identified that reuse had a 68.2 percent similarity to the ideal element followed by refurbish and recycle both with a 54.5 percent similarity. Refurbish had a 59.1 percent similarity to recycle and was the highest of the nongrounding elements, whilst reuse had a 50 percent similarity to both refurbish and recycle, which was the lowest of the non-grounding elements. This suggests that the participant views reuse as the most ideal thing to do with a used mobile phone compared to both refurbishing and recycling, whilst refurbishing is perceived as more similar to recycling. During analysis of the repgrid constructs, three of the elicited constructs needed to be reversed in order to achieve the highest possible percentage similarity across constructs. Two constructs had a 100 percent similarity with the overall construct. These constructs were related to the subcategory of having a spare and reflected the participants desire to disassemble old phones for their parts along with the need to avoiding wasting a product that could be reused. A construct that reflected concern for the environment only had an 80 percent similarity to the overall construct. 


\section{Participant 8}

Participant 8 is aged between 35 and 55 with an income greater than $\$ 100,000$. They are employed full time as a manager within the legal sector and their highest academic achievement was at the postgraduate level. Analysis of the percentage similarity across elements identified both refurbish and recycle had an 86.5 percent similarity whilst reuse had an 82.7 percent similarity with the ideal element. Refurbish was seen as similar to recycle with a 96.2 percent similarity, and was the highest of the non-grounding elements, whilst reuse had an 88.5 percent similarity to recycle, which was the lowest of the non-grounding elements. This suggests that the participant sees both refurbishing and recycling as the best way to manage used mobile phones, and is reflected in the participant view that refurbishing and recycling are the most similar. During analysis of the repgrid constructs, one of the elicited constructs needed to be reversed in order to achieve the highest possible percentage similarity across constructs. Seven constructs were closely associated with the overall construct with a 70 percent similarity. These constructs related to subcategories associated with the age of technology, environmental awareness, social norm/culture, functioning, and income and reflected ideas of reliance on technology, priorities, and family.

\section{Participant 9}

Participant 9 is aged between 35 and 55 with an income range of $\$ 25,000$ to $\$ 50,000$. They are employed part time as a nurse within the health sector and their highest academic achievement was at the undergraduate level. Analysis of the percentage similarity across elements identified that reuse had the highest percentage similarity at 84.1 percent followed by recycle at 81.8 percent and refurbish at 75 percent. Reuse was aligned with recycle with an 88.6 percent similarity, and was the highest of the non-grounding elements, whilst refurbish and recycle had an 84.1 percent similarity, which was the lowest of the non-grounding elements. This suggests that the participant sees reuse as the best approach to managing used mobile phones whilst reuse is seen as slightly more similar to recycling than refurbishing is to recycling. Two constructs were closely associated with the overall construct with a 90 percent similarity and they were related to the subcategory residual value whilst a construct that reflected concern for the environment only had a 60 percent similarity to the overall construct. This suggests this participant places more importance on the residual value in a used mobile phone than concern for the environment. 


\section{Participant 10}

Participant 10 is aged between 18 and 34 with an income range of $\$ 25,000$ to $\$ 50,000$. They are a full time student and their highest academic achievement was a high school qualification. Analysis of the percentage similarity between elements identified that reuse had an 86.4 percent similarity to the ideal element followed by both recycle and refurbish at 84.1 percent. Reuse was aligned with refurbish with an 88.6 percent similarity and was the highest of the non-grounding elements, whilst refurbish had a 72.7 percent similarity with recycle, which was the lowest of the non-grounding elements. This suggests that the participant sees reusing as a more ideal way to manage used mobile phones and that reuse is perceived as similar to refurbishing. Four constructs were closely associated with the overall construct with a 90 percent similarity and were related to subcategories of social norm/ culture and environmental awareness. This suggests the participant, has concerns in terms of how they influence their social circle as well as their impact on the environment.

\section{Participant 11}

Participant 11 is aged between 35 and 55 with an income range greater than $\$ 100,000$. They are employed full time lawyer within the legal sector and their highest academic achievement was at the postgraduate level. A comparison of the percentage similarity between the elements indicated that recycle was aligned with the ideal element with a 90.9 percent similarity followed by reuse and refurbishing, both with a 70.5 percent similarity. Refurbish was aligned with recycle with a 79.5 percent similarity, and was the highest of the non-grounding elements, whilst reuse had a 63.6 percent similarity to refurbish, and was the lowest of the non-grounding elements. This indicates that for this participant, recycling a used mobile phone is seen as more ideal than both reusing and refurbishing, whilst recycling is more aligned with refurbishing. Analysis of the percentage similarity across constructs resulted in three constructs being reversed. One construct had an 80 percent similarity to the overall construct and was related to the subcategory the age of technology whilst three constructs that reflected concern for the environment had a 70 percent similarity to the overall construct. This suggests the age of technology is slightly more important to this participant than the environment.

\section{Participant 12}

Participant 12 is aged between 18 and 34 with an income range of $\$ 50,000$ and $\$ 75,000$. They are employed full time as an analyst within the justice sector and their highest academic achievement 
was at the postgraduate level. A comparison of the percentage similarity between elements identified that reuse had a 97.7 percent similarity with the ideal element followed by refurbish at 77.3 percent and recycle at 72.7 percent. Refurbish had an 81.8 percent similarity with recycle and was the highest of the non-grounding elements, whilst reuse only had a 70.5 percent similarity with recycle which was the lowest of the non-grounding elements. This suggests the participant sees reuse as the more ideal way to manage used mobile phones and sees refurbishing and recycling as the most similar. Four constructs all had a 100 percent similarity to the overall construct and were all related to the subcategory environmental awareness, which suggests the participant has a strong concern for the environment in terms of managing used mobile phones.

\section{Participant Summary}

The following table (Table 2) provides a summary of the demographic information provided by each participant and includes a brief summary of the important aspects of their repgrids analysis. 


\begin{tabular}{|c|c|c|c|c|c|c|c|c|c|}
\hline Participant & Age & Gender & Income & Education & Emplogment & Position & $\begin{array}{l}\text { Most ideal } \\
\text { element }\end{array}$ & $\begin{array}{l}\text { Most similar } \\
\text { elements }\end{array}$ & Most similar to overall construct \\
\hline 1 & $18-34$ & $\mathrm{~F}$ & $\$ 50,000-\$ 75,000$ & High School & Full time & Legal support leader & Recycle & $\begin{array}{l}\text { Reuse and } \\
\text { refurbish }\end{array}$ & $\begin{array}{l}\text { Concern for the environment, how impact on } \\
\text { environment will affect them. }\end{array}$ \\
\hline 2 & $35-55$ & $\mathrm{~F}$ & $\$ 25,000-\$ 50,000$ & Postgraduate & Part time & Midwife & Recycle & $\begin{array}{l}\text { Reuse and } \\
\text { recycle }\end{array}$ & $\begin{array}{l}\text { Concern for the environment, well informed with } \\
\text { money, lack of need for the latest technology, role } \\
\text { model. }\end{array}$ \\
\hline 3 & $18-34$ & $\mathrm{~F}$ & $\$ 50,000-\$ 75,000$ & Undergraduate & Full time & Administrator & Recycle & $\begin{array}{l}\text { Reuse and } \\
\text { refurbish }\end{array}$ & $\begin{array}{l}\text { Concern for the environment, avoid waste, more } \\
\text { information on recyling needed. }\end{array}$ \\
\hline 4 & 74-91 & $\mathrm{F}$ & $\$ 50,000-\$ 75,000$ & High School & Retired & - & Recycle & $\begin{array}{l}\text { Reuse and } \\
\text { refurbish }\end{array}$ & $\begin{array}{l}\text { Concern for the environment, healthy environment for } \\
\text { family, avoid waste. }\end{array}$ \\
\hline 5 & $35-55$ & $\mathrm{~F}$ & $\$ 25,000-\$ 50,000$ & Undergraduate & Part time & Nurse & Recycle & $\begin{array}{l}\text { Recycle and } \\
\text { refurbish }\end{array}$ & $\begin{array}{l}\text { Concern for the environment, sombody else should } \\
\text { benefit. }\end{array}$ \\
\hline 6 & $35-55$ & $M$ & Above $\$ 100,000$ & Trade cert & Full time & Soldier & Reuse & $\begin{array}{l}\text { Reuse and } \\
\text { recycle }\end{array}$ & Repairing mobile phones. \\
\hline 7 & $35-55$ & $M$ & $\$ 50,000-\$ 75,000$ & Trade cert & Full time & FitterdTurner & Reuse & $\begin{array}{l}\text { Recycle and } \\
\text { refurbish }\end{array}$ & Disassembling used phones for parts. \\
\hline 8 & $35-55$ & $M$ & Above $\$ 100,000$ & Postgraduate & Full time & Manager & $\begin{array}{l}\text { Reuse and } \\
\text { recycle }\end{array}$ & $\begin{array}{l}\text { Recycle and } \\
\text { refurbish }\end{array}$ & $\begin{array}{l}\text { Concern for the environment, reliance on technology. } \\
\text { priorities, and family. }\end{array}$ \\
\hline 9 & $35-55$ & $\mathrm{~F}$ & $\$ 0-\$ 25,000$ & Undergraduate & Part time & Nurse & Reuse & $\begin{array}{l}\text { Reuse and } \\
\text { recycle }\end{array}$ & Residual value and saving money \\
\hline 10 & $18-34$ & $\mathrm{~F}$ & $\$ 25,000-\$ 50,000$ & High School & Student & - & Reuse & $\begin{array}{l}\text { Reuse and } \\
\text { refurbish }\end{array}$ & Concern for the environment, be a good influence. \\
\hline 11 & $35-55$ & $M$ & Above $\$ 100,000$ & Postgraduate & Full time & Lawyer & Recycle & $\begin{array}{l}\text { Recycle and } \\
\text { refurbish }\end{array}$ & The age of the technology \\
\hline 12 & $18-34$ & $\mathrm{~F}$ & $\$ 50,000-\$ 75,000$ & Postgraduate & Full time & Analyst & Reuse & $\begin{array}{l}\text { Recycle and } \\
\text { refurbish }\end{array}$ & $\begin{array}{l}\text { Concern for the environment, the age of the } \\
\text { technology, social good }\end{array}$ \\
\hline
\end{tabular}

Table 2. Participant summary 


\subsection{Group analysis}

\subsubsection{Element analysis (Reuse, Refurbish, Recycle)}

As with individuals, it is possible to compare the percentage similarity across elements for the group of participants because each participant was presented with the same set of elements. Therefore, the following section examines the results of the element analysis from a group perspective.

All but one participant had a 73 percent similarity or greater between recycling and the ideal element. The one participants where recycling had a slightly higher percentage similarity to the worst element, can be explained by this participant's particular focus on being able to reuse a product for his own use as opposed to sending it away for recycling where there is some perceived loss of residual value. Both reuse and refurbishing had a 50 percent or greater percentage similarity to the ideal element across all but one participant. The only participant that indicated a low percentage similarity was participant 5 where reuse had 28.6 percent similarity to the ideal element and a 75 percent similarity to the worst thing to do. When it came to reuse, these figures were noticeably distinct from the other participants' responses but appear to reflect participant 5's strong positive feelings towards recycling and negative feelings associated with storing because of the associated lost value in terms of a declining technology that is unlikely to be used, as well as the perceived clutter, it generates. The participants concerns in regards clutter suggest that the small size of a mobile phone does not necessarily prevent it from being associated with the idea of unnecessary storage for some people. However, to give that some context only one person in this study raised the idea of storage creating clutter and for them size only had a low level of importance.

Three participants had identical rating structures when it came to the percentage similarity of the elements when using just using a high, intermediate, or low rating whilst ignoring the exact values. For this process, a high rating was defined as 67 percent to 100 percent, intermediate 34 percent to 66 percent, and low 0 percent to 33 percent. The resulting analysis indicated reuse, refurbishing and recycling all had a high level of similarity to the ideal element. This appears to indicate that for participants 9,10 and 12 they view reuse, refurbishing and recycling all as highly similar to the ideal thing to do whilst having minor distinctions as to which is more ideal at an individual level. They also had comparable levels of percentage similarity across all the non-grounding constructs. It is not clear if there was any reason for this matching structure, or for the apparent stronger polarity in 
terms of these participants consistent high percentage similarity to the ideal thing to do and consistent low percentage similarity to the worst thing to do, as the only known commonality across all three participants was that they were female. There may be a more explanatory reason to be found in areas of demographic data that were not collected, however, on a superficial level, it may suggest that in some instances females may be more emotive in terms of their expression of when something is ideal and when it is not. However, it is important to note that this apparent polarity was absent in the male participants and did not extend across all the female participants.

In contrast, participants 7 and 8 had noticeably dissimilar percentage similarity structures. Participant 7 was consistently at the intermediate level in terms of percentage similarity in all but one measure whilst participant 8 was at the high percentage similarity level in all but one measure. The only percentage similarity that matched in terms of it being at a high level was that between reuse and the ideal thing to do. Both participants were male with differing levels of education, income, and age. One participant's employment was more practically focused whilst the other held a professional managerial position. Participant 7 appeared to be more focused on deconstruction and reusing parts as a form of entertainment whilst participant 8 was more focused on reuse in terms of timesaving and its impact on priorities and family as well as having a more pragmatic view of potential issues. These different areas of concern could be a reflection of their different levels of life experience suggested by their age range differences or it could be related to their level of education. However, whilst demographic information such as age and education may play a role in terms of the importance these participants placed on factors presented in the $3 \mathrm{R}$ model, there does not appear to be a straightforward connection in terms of the effect on their core beliefs.

An examination of the combination of elements presented during the triadic elicitation process identified that three participants identified the grounding elements of worst and ideal as being similar for one of the construct elicitations. This was identified because these participants all had similar responses during part of the interview in regards the ideal and worst elements. They all suggested that these approaches were based on opinion rather than fact. All of these participants appeared to exhibit a higher level of confidence during the interview process and when providing constructs during elicitation. This suggests that these participants appear to be confident in challenging what they perceive as just "opinion" and may present a problem if they were presented with educational material that challenged on own internal beliefs. However, it should be noted that 
not all participants who appeared confident during the interview process had the same type of response when considering the ideal and worst elements.

Overall the results of the element analysis suggests that in general, all three approaches, reuse, refurbishing and recycling appear to be perceived as being good ways of managing used mobile phones, but to varying degrees. However, whilst all three are generally perceived to be good, participants appeared to prefer either reuse or recycling out of the three approaches. The alignment of reuse towards the ideal thing to do is potentially problematic considering the long-term, environmental ramifications, of storing used mobile phones. In general, the perception that reuse and therefore storing a used mobile phone is closer to the ideal thing to do provides some support to Baxter and Gram-Hanssen (2016) where it was suggested that mobile phones may be stored because people perceive their actions as being environmentally neutral. However, the results of this study suggest that the environmentally neutral position may be too conservative and that in fact people perceive storing as a positive outcome in terms of the environment.

\subsubsection{Honey's content analysis}

Although Jankowicz (2005) did not adopt a ranking system when explaining Honey's content analysis a ranking system was adopted in this research (Appendix 2). This was done because a ranking system can be used in content analysis when comparing how frequently certain codes occur (Hsieh \& Shannon, 2005). Since using Honey's content analysis had already created a frequency count, the next logical step was to add a ranking order. This ranking related to how many codes were coded to a specific subcategory, category or combination of subcategory-category. The frequency of the codes found under within a specific area was used as an indicator of its importance to the participants as a group. As previously mentioned, for the purposes of this study some terms have the same meaning, for instance, subcategory and factor (Please refer to table 1).

Constructs were assigned to subcategories, but several subcategories had links to more than one category. As a result, the structure of the content analysis table (Appendix 2) prevented the easy assignment of a ranking to each of the categories. As a result, the content analysis table was compressed and realigned so that subcategories, which were related to multiple categories, were now split by category. This meant that categories that had been previously split by subcategory could now be combined. The result was that it was possible to give each category a ranking (Figure 13). This 
means the results section uses a combination of Honey's content analysis table (Appendix 2) and the result of the compressed table (Figure 13). The main difference between them is that figure 13 has the category rankings where appendix 2 does not, however, appendix 2 has the constructs, H-I-L value and percentage similarity to the overall construct.

It should also be noted that whilst some constructs may appear obscure, because of the limited ability to capture context in a few words, all constructs were confirmed by the researcher with the participant before capture, and their relative context considered during coding.

\section{Environmental awareness}

Of all the potential subcategories, environmental awareness was coded significantly more times than any other subcategory and had 39 percent of constructs coded to it. It was also identified to varying degrees across all interviews and had an overall subcategory ranking of one, which means it was the single most important subcategory of all subcategories identified before any splitting. However, because environmental awareness is suggested to have a relationship with three categories storing, purchasing refurbished, and engagement (Figure 12) the results of the subcategory-category combination were also reviewed.

It was identified that the combination of environmental awareness and storing had 10 percent of the constructs coded to it, giving it a subcategory-category ranking of three (Figure 13). The percentage similarity to the overall construct prior to H-I-L analysis ranged between 0 percent and 100 percent with only one having less than a 70 percent similarity (Appendix 2). This suggests that in general most participants appeared to associate environmental awareness and storing fairly strongly with used mobile phones. The percentage similarity and H-I-L ratings were then re-examined once the constructs that expressed the same idea but had different H-I-L ratings were removed, as suggested by Jankowicz (2005). An example of the H-I-L process can be seen in figure 11. Analysis of the percentage similarity to the overall construct showed that, as a group, all constructs associated with this subcategory/category combination had percentage similarities of between 70 and 90 percent to the overall construct. There also appeared to be some consistency across the constructs H-I-L ratings with constructs exhibiting either a high or an intermediate rating whilst none exhibited a low H-I-L rating. 


\begin{tabular}{|c|c|c|c|c|}
\hline Construct & HIL $\%$ & Subcategory & Category & Outcome \\
\hline 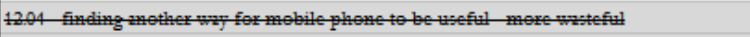 & H $100 \%$ & 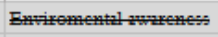 & Stering & Reuse \\
\hline 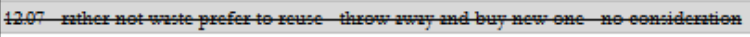 & H $100 \%$ & 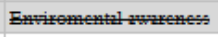 & Stering & Restros \\
\hline 5.06 environmentally friendly - environmentally unfriendly $(R)$ & H $90 \%$ & Enviromental awzreness & Storing & Reuse \\
\hline 10.08 I want to do good things - not caring & H $90 \%$ & Enviromental awareness & Storing & Reuse \\
\hline 1208 best thing to do is reuse it jut threw it in the twh is the wert thing to do & $+90 \%$ & 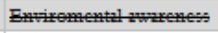 & Stering & Reuse \\
\hline 2.07 good utilization - harmful & H $80 \%$ & Enviromental awareness & Storing & Reuse \\
\hline 7.04 you can do anything - can't do anything $(R)$ & H $80 \%$ & Enviromental awareness & Storing & Reuse \\
\hline 6.01 finite source - depletion & $180 \%$ & Enviromental awareness & Storing & Reuse \\
\hline 1.1 because I care - you should care & $180 \%$ & Enviromental awareness & Storing & Reuse \\
\hline 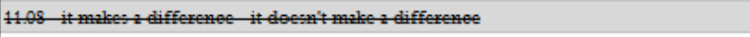 & $H^{\prime} 700 \%$ & 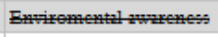 & Stering & Restl \\
\hline 4.09 best use of product - reuse & H $70 \%$ & Enviromental awareness & Storing & Reuse \\
\hline 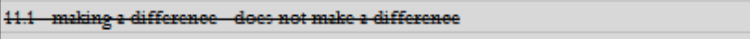 & $H^{\prime} 700 \%$ & 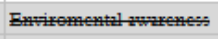 & Stering & Rewe \\
\hline 11.01 gut fecling better decision $(R)$ & Qts & 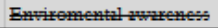 & Storing & Rewse \\
\hline
\end{tabular}

Figure 11. Example H-I-L analysis

Overall, the subcategory-category ranking of three indicates the combination is an important one from a group perspective whilst the higher percentage similarity indicates participants strongly associated it the idea of used mobile phones. Finally, the H-I-L indices indicated an intermediate to high level of relative importance to each individual within the wider group.

Analysis of the combination of environmental awareness and engagement identified that this subcategory/category combination had 28 percent of the constructs coded against, with a subcategory-category ranking of one. The percentage similarity to the overall construct ranged from between 10 to 100 percent and accounted for 10 out of 12 participants (Appendix 2). However, following H-I-L analysis, only a few constructs remained once conflicting constructs were removed which prevented analysis of the relativistic nature of the percentage similarity from individual participants' perspective. Overall, the subcategory-category ranking indicates that from a group perspective it was the most important subcategory-category combination and represented the majority of the group. However, the wide range of percentage similarity ratings indicates that there was a broad range in the way participants associated it with the idea of used mobile phones. The results of the H-I-L analysis also seem to suggest that when there are several core constructs expressed by individuals in relation to environmental awareness and engagement, each core construct may not be consistently important to the individual.

Finally, the combination of environmental awareness and engagement only had 1 percent of the constructs coded to it. This gave it a subcategory-category ranking of 17 and only represented one participant (Figure 13). This ranking combined with its 90 percent percentage similarity rating to the overall construct and intermediate H-I-L indices (Appendix 2) indicates that even though the 
participant who mentioned it strongly associated the subcategory-category with used mobile phones, it only appeared to hold an intermediate level of importance.

\section{Social Norm/Culture}

Social norm/culture had 20 percent of the constructs at the subcategory level coded to it, which gave it a subcategory ranking of two (Figure 13), which suggests it was the second most important subcategory before splitting. Unlike environmental awareness, however, social norm/culture was only coded in 10 of the participants' interviews, although this still represents the majority of participants. As with environmental awareness, social norm/culture was suggested to have a relationship with three categories engagement, on giving, and purchasing refurbished (Figure 12). Analysis of each these subcategory-category combinations identified that social norm/culture when associated with engagement had 11 percent of the constructs coded against it and a subcategorycategory ranking of two (Figure 13). The percentage similarity had a range of 50 to 90 percent prior to H-I-L analysis (Appendix 2), however, H-I-L analysis resulted in the number of represented participants dropping from nine to five; the remaining H-I-L indices all had an intermediate to high level. Overall, this indicates that once social norm/culture was split amongst its related categories it was the second most important subcategory-category combination, and it was also associated with managing used mobile phones at a mid to high level by participants and from an individual perspective had a mid to high level of importance.

The combination of social norm/culture and on giving had 5 percent of constructs coded against it and a subcategory-category ranking of six (Figure 13). Analysis of the percentage similarity to the overall construct prior to H-I-L analysis indicated a range of 70 percent to 90 percent and was reflective of four participants constructs (Appendix 2). However, following H-I-L analysis only one construct remained which prevented a broad analysis of the relativistic nature of the percentage similarity and therefore establishing the relative importance to each individual across the group. However, the one individual presented by the remaining construct indicated a 70 percent similarity to the overall construct but only an intermediate level on the H-I-L indices. Overall, the combination of social norm/culture and on giving with its subcategory-category ranking of six suggests that it was the sixth most important subcategory-category and prior to H-I-L analysis the percentage similarity suggests it was fairly strongly associated with used mobile phones. The single remaining participant post H-I-L analysis suggests, they associated social norm/culture and on 
giving relatively highly with used mobile phones but only felt it was at an intermediate level of importance to them.

The combination of social norm/culture and purchasing refurbished had 3 percent of the constructs coded against it and a subcategory-category ranking of 10 (Figure 13). Analysis of the percentage similarity prior to H-I-L analysis indicated a range of 50 percent to 90 percent and was reflective of three participants constructs (Appendix 2). After H-I-L analysis was conducted, the remaining constructs consisted of two participants. However, although the 50 percent to 90 percent similarity to the overall construct remained there was no consistency across the H-I-L indices with each level of indices represented in the results. Overall, this suggests that this subcategory-category has a lower level of importance from a group perspective, whilst being mildly to strongly associated with managing old mobile phones, with a level of importance that varied significantly across individuals.

\section{Age of technology}

The subcategory Age of technology had 7 percent of the constructs coded to it, which gave it a subcategory ranking of three, which suggests it was the third most important subcategory before any splitting. However, the age of technology was only linked to one category, purchasing refurbished which resulted in subcategory/category ranking of four (Figure 13). Analysis of the percentage similarity prior to H-I-L analysis indicated a range of 60 to 90 percent (Appendix 2), however, H-I-L analysis resulted in the number of represented participants dropping from seven to six; with the remaining H-I-L indices all having an intermediate to high level. However, it should be noted that only one participant was responsible for the intermediate H-I-L rating on two constructs. Overall, this suggests this subcategory-category was fairly important, whilst participants felt it was quite strongly associated with managing old mobile phones and it had a mid to high level of relative importance to them individually. It should be noted though that for some participants, the age of the technology was not necessarily perceived as a negative, rather it could be perceived as positive if it aligned with their requirements.

\section{Functioning}

This subcategory had 6 percent of the constructs at the subcategory level coded to it, which gave it a subcategory ranking of four (Figure 13). This suggests that functioning was the fourth most important subcategory for half of the participants. The functioning subcategory was only linked to 
one category, storing, which resulted in a subcategory-category ranking of six. Analysis of the percentage similarity to the overall construct prior to H-I-L analysis indicated a range of 20 to 100 percent (Appendix 2), which changed to 20 to 90 percent post analysis. However, once H-I-L analysis was completed the number of represented participants dropping from six to three, with all the remaining H-I-L indices having no consistency in terms of their level. This suggests that functioning, when considered in terms of its relationship to storing was the fifth most important subcategory-category whilst there was a broad range in how much people associated it with the idea of managing used mobile phones. In addition, whilst the H-I-L analysis did halve the number of participants that were represented, there was still a significantly broad range of H-I-L indices indicating there was no consistency in terms of its relative importance to participants.

\section{Residual value}

This subcategory had 5 percent of the constructs coded to it, which gave it a subcategory ranking of five whilst the subcategory was only linked to one category, storing, which resulted a subcategorycategory ranking of seven (Figure 13). Analysis of the percentage similarity to the overall construct prior to H-I-L analysis indicated a range of 20 to 90 percent (Appendix 2), while H-I-L analysis resulted in the number of represented participants dropping from three to one. Whilst preventing analysis of the importance to each individual relatively across the group, the remaining construct indicated that one participant felt that residual value and storing was only weakly related to managing used mobile phones and that the relative importance to them was low. Overall, this suggests, whilst there appears to be a mid level of importance attributed to both the subcategory and subcategory/category combination at the group level, at the individual level there was significant variance in the how much it was linked with managing used mobile phones.

\section{Spare}

This subcategory had 5 percent of the constructs coded to it, which gave it a subcategory ranking of six whilst the subcategory was only linked to one high level category, storing, which resulted a subcategory/category ranking of eight (Figure 13). Even though these rankings were only reflective of a single participant, a H-I-L analysis was undertaken to determine if there was any ambiguity in the participants coded constructs. The result indicated there was strong ambiguity across all constructs indicating there was no consistency in their relative importance. Overall, this suggests that 11 of the participants did not consider the idea of a spare mobile phone when it comes to storing 
used mobile phones and that the importance to one individual was responsible for the overall rankings.

\section{Perception}

This subcategory had 4 percent of the constructs coded to it, which gave it a subcategory ranking of seven and since the subcategory was only linked to one high level category the combination received subcategory/category ranking of nine (Figure 13). Prior to H-I-L analysis the percentage similarity to the overall construct ranged from 50 percent to 90 percent (Appendix 2) whilst H-I-L analysis resulted in the removal of four constructs and two participants that left just one construct representing one participant. This prevented relativistic analysis of the percentage similarity to determine if there is any consistency across participants, however, for the single participant the idea of the subcategory/category combination was both highly associated with managing an old mobile phone and highly important indicate by the high H-I-L indices. Overall, this suggests the subcategory, on its own and when associated with purchasing a refurbished mobile phone, has a mid-level of importance, however; the percentage similarity to the overall construct indicates a mid to high level of association with managing old mobile phones.

\section{Other coded subcategories}

Knowledge, price and income were all subcategories that had between two and three percent of the constructs resulting in subcategory rankings of between eight and nine and as a group represented nine of the participants. These subcategories were all associated with purchasing a refurbished mobile phone and when considered as a subcategory/category combination their subcategory/category ratings ranged between 10 and 11 (Figure 13). They all had a variety of percentage similarities to the overall construct (Appendix 2) indicating there was significant variation in the way participants viewed how closely related these subcategories were to managing old mobile phones. H-I-L analysis also indicated variety in terms of their relative importance to individuals when analysis did not remove conflicting constructs.

Size, ease, and trust (data) were all subcategories that had between one to two percent of the constructs resulting in subcategory rankings of between 11 and 13, with 13 being the lowest level ranking for a subcategory that had associated constructs (Figure 13). Size was associated with one category, storing, whilst ease was associated with both on giving or recycling. Trust (data) was 
associated with storing, on giving and engaging. Of trust (data), only the combination of trust (data) and on giving had a construct coded against it. As a group, these subcategories represented four participants but each subcategory was generally only associated with a single participant. When considered in combination with their associated categories the subcategory/category ranking was between 14 and 16 (Figure 13). Analysis of the percentage similarity to the overall construct prior to H-I-L analysis indicated a range of 60 percent to 90 percent (Appendix 2) which indicated they were perceived as having a mid to high level of association with managing used mobile phones depending on the participant. However, the limited number of constructs prevented any in-depth informative H-I-L analysis. Only one construct appeared to be relatively significant as it related to the size of mobile phones and indicated that the one participant who did consider it associated with used mobile phones only felt it was of low importance.

\section{Other non-coded subcategories}

Several subcategories identified in the original model were not coded against any constructs. Some of these subcategories such as experience and regulations were only linked to one high level category. Whilst these subcategories appear to stand out because they had no associated constructs it does not preclude them from being retained in the model, however, where subcategories were removed, the reasoning for their removal was discussed.

\subsubsection{Model additions}

\section{Macro-economic}

During repgrid coding, a new subcategory emerged that had not been previously considered. This subcategory reflects the idea of macro level economic improvement through the establishment of new jobs that are created because of the recycling process. The subcategory only emerged from a single participant, however, it was a core consideration for this person and a motivating factor to engage with recycling. Whilst having a low subcategory ranking of 11 (Figure 13) it did appear to fit with other low-level subcategories. When considered in terms of its link to recycling, the subcategory/category combination ranking was 14 (Figure 13). Prior to H-I-L analysis, the percentage similarity of the two constructs to the overall construct was 50 percent to 80 percent

(Appendix 2), however, H-I-L analysis resulted in both constructs being removed because they had conflicting H-I-L indices. 


\section{New environmental awareness link}

During the process of repgrid coding, an existing subcategory appeared to be directly linked to a category in a way that was not previously captured in the original model. Environmental awareness whilst already having linkages to a couple of categories was not a subcategory that was originally identified as affecting the purchasing refurbished category. Whilst this participant appeared to be motivated to purchase a refurbished mobile phone over a new mobile phone because it was associated with meeting their needs, they also appeared to have a strong sense of environmental awareness, which meant it needed to be added to the model (Figure 12).

\section{Categories}

Overall, engagements ranking of one indicates it is the most important category even though only three of its related factors had constructs coded to them, this is followed by storing, purchasing refurbished and on giving.

\section{Summary}

In summary, it appears as though both subcategories and categories, when viewed either individually or in combination, have varying degrees of influence across the $3 \mathrm{R}$ model. The implications of these results need to be discussed, including their impact on the original $3 \mathrm{R}$ model and any potential adjustments that are required. 


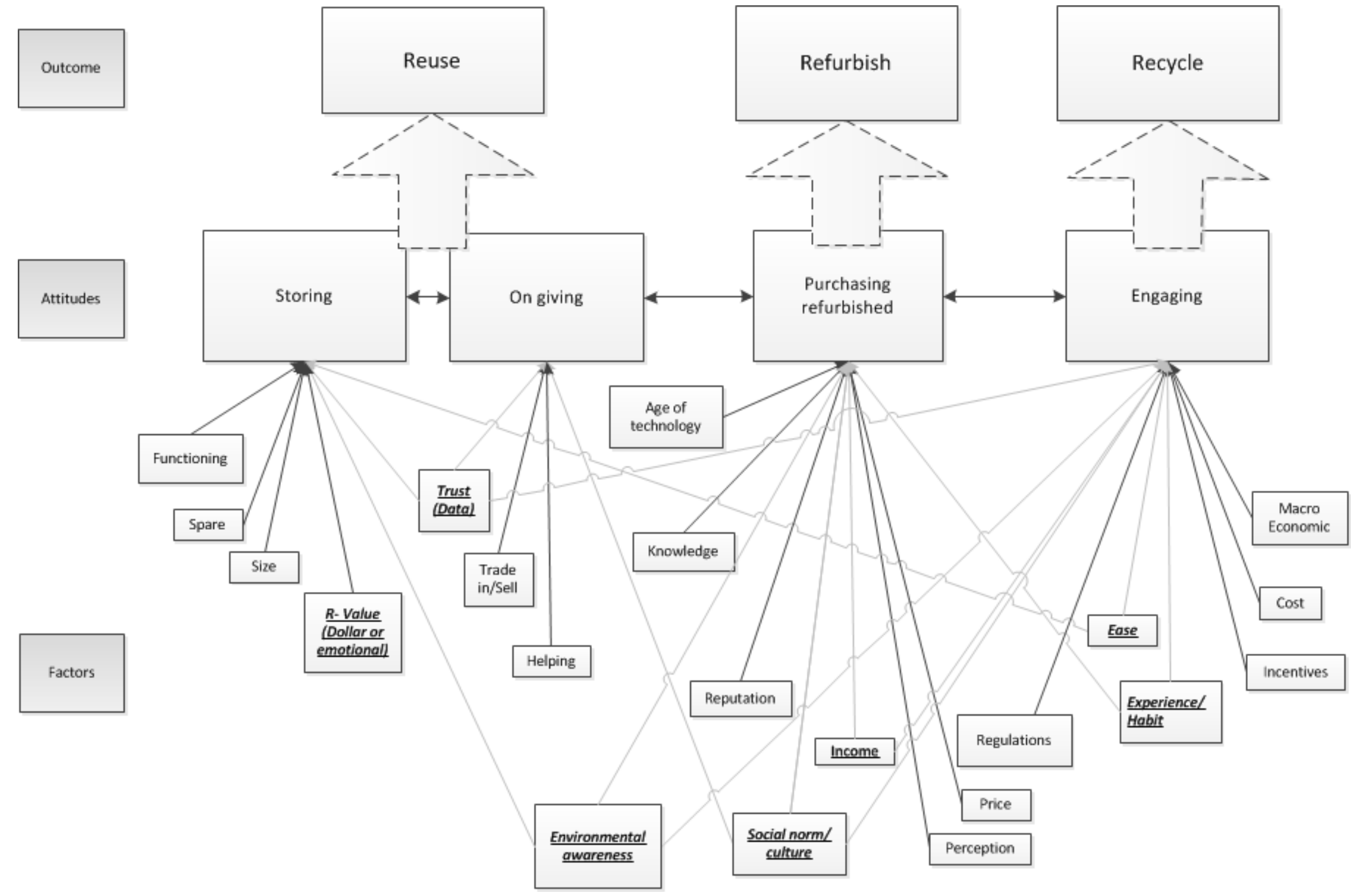




\begin{tabular}{|c|c|c|c|c|c|c|c|c|c|c|c|c|c|c|c|c|c|c|c|c|c|c|c|c|c|c|c|c|c|}
\hline & \multicolumn{10}{|c|}{ Reuse } & \multicolumn{9}{|c|}{ Refurbish } & \multicolumn{10}{|c|}{ Recycle } \\
\hline Category ranking & \multicolumn{7}{|c|}{2} & \multicolumn{3}{|c|}{4} & \multicolumn{9}{|c|}{3} & \multicolumn{10}{|c|}{1} \\
\hline $\begin{array}{l}\text { Categories } \\
\end{array}$ & \multicolumn{7}{|c|}{ Storing } & \multicolumn{3}{|c|}{ On giving } & \multicolumn{9}{|c|}{ Purchasing refurbished } & \multicolumn{10}{|c|}{ Engagement } \\
\hline $\begin{array}{c}\text { Subcategory/Category } \\
\text { ranking }\end{array}$ & 16 & 8 & 14 & 3 & 19 & 6 & 7 & 19 & 4 & 16 & 19 & 12 & 4 & 16 & 10 & 12 & 10 & 9 & 19 & 19 & 19 & 2 & 19 & 19 & 19 & 19 & 19 & 1 & 14 \\
\hline $\begin{array}{l}\text { Subcategories split by } \\
\text { category }\end{array}$ & ชั้ & 气ั & \&ै & 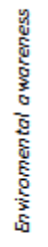 & 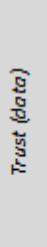 & 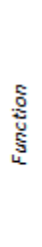 & $\frac{\frac{5}{5}}{\alpha^{\prime}}$ & 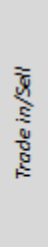 & 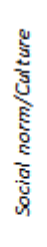 & 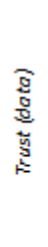 & 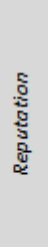 & 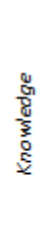 & 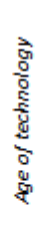 & 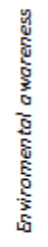 & 气ू้ & है & 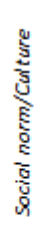 & 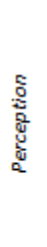 & 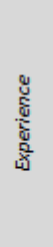 & 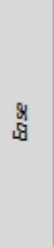 & 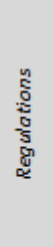 & 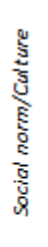 & 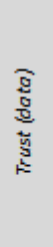 & 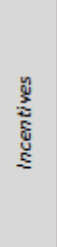 & 氙 & 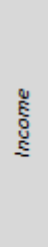 & 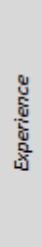 & 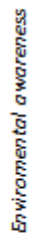 & 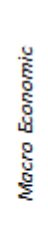 \\
\hline
\end{tabular}

\begin{tabular}{|l|c|}
\hline \multicolumn{1}{|c|}{$\begin{array}{c}\text { Subcategory rankings } \\
\text { (Before split) }\end{array}$} & \\
\cline { 2 - 2 } Enviromental awareness & Rank \\
\hline Social norm/Culture & 1 \\
\hline Age oftechnology & 2 \\
\hline Function & 3 \\
\hline R_Value & 4 \\
\hline Spare & 5 \\
\hline Perception & 6 \\
\hline Price & 7 \\
\hline Income & 8 \\
\hline Knowledge & 9 \\
\hline Ease & 9 \\
\hline Macro Economic & 11 \\
\hline Size & 11 \\
\hline Trust (data) & 13 \\
\hline Trade in/Sell & 13 \\
\hline Reputation & 14 \\
\hline Experience & 14 \\
\hline Regulations & 14 \\
\hline Incentives & 14 \\
\hline Cost & 14 \\
\hline
\end{tabular}

Figure 13. The 3R model (Content analysis applied)

Note: Subcategories shaded in grey received no coded constructs 


\section{Discussion}

This study has attempted to determine what underlying factors impact people's attitudes, and how those attitudes can affect the sustainable management of used mobile phones. It appears, based on the repgrid element analysis, participants generally see reuse, purchasing refurbished mobile phones and recycling as positive ways to manage used mobile phones, as a result, the following section will discuss the both the factors and attitudes presented in the $3 \mathrm{R}$ model that lead to these outcomes. As previously mentioned, for the purposes of this study some terms have the same meaning, for instance, subcategory and factor (Please refer to table 1).

\section{Environmental awareness}

There have been conflicting suggestions that consumers with greater environmental awareness are more likely to engage in recycling (Leiserowitz et al., 2006; Welfens et al., 2015; Yin et al., 2014) or conversely higher levels of environmental awareness makes no difference (Baxter \& Gram-Hanssen, 2016; Wang et al., 2011). The results of this study seem to indicate there is a link between environmental awareness and engagement as it was the top subcategory/category ranking in this study (Figure 13). However, the degree to which people feel enviromental awareness is important appears to vary across individuals. An important implication of these results is that it identifies that people's core beliefs reflect a need to be environmentally aware and recycle old mobile phones, which is important because people's core beliefs are the most difficult beliefs to change. Therefore, the challenge of raising environmental awareness using educational programmes does not face the task of having to create or change existing core beliefs, however, there is still a need to leverage and amplify existing beliefs in order to be effective. By raising this relative level of importance, it may be possible stimulate behavioral changes that reflect those heightened attitudes.

Environmental awareness was also linked with storing old mobile phones and was one of the top three subcategory/category rankings in this study (Figure 13). When considered together with the suggestion that storing is perceived to be closer to the ideal thing to do than the worst it provides additional support that concern for the enviroment plays a role in peoples attitudes towards storing used mobile phones. Several other studies (Ongondo \& Williams, 2011a; Speake \& Yangke, 2015; Suckling \& Lee, 2015; Welfens et al., 2013; Wilhelm et al., 2015; Ylä-Mella et al., 2015) have 
identified that people tend to store their used mobile phones. As suggested previously, the problem is that storing a used mobile phone may not be the most environmentally appropriate course of action because of the potential loss of future use (Ylä-Mella et al., 2015) and the risk that stored mobile phones are more likely to end up in the household rubbish (Jang \& Kim, 2010). This is because technology does become obsolete and this can occur while it is being stored and eventually the old phone still needs to be disposed of. Finally, the impact of environmental awareness when considered in the context of purchasing refurbished mobile phones does not appear to be significant. As a result, environmental awareness appears to be an important consideration across two areas, storing and engagement. It is therefore suggested educational and take back programmes should target people's perceptions that storing a used mobile phone is an environmentally friendly option and provide information to suggest the contrary is true, whilst at the same time continuing to encourage recycling. Programmes should also avoid giving too much emphasis to promoting the purchasing of refurbished mobile phones from an environmental awareness perspective as this may only have limited impact given its low level of importance to participants.

\section{Social norm/Culture}

This study seems to indicate that the social norms and culture that surrounds peoples lives is the second most important subcategory that affects attitudes towards engaging with recycling, and is significantly more important than any other remaining subcategory under engagement (Figure 13). This supports the suggestion by Hansmann et al. (2006) that both the social and cultural background of individuals appears to impact behaviour, as well as Welfens et al. (2015) who suggested that educational messages needed to target social groups in order to be effective. The use of social media to deliver targeted educational programmes may be a good approach because of the speed and the levels of distribution social networks can achieve at a relatively low cost compared to more traditional modes of delivery such as television and radio advertising. Educational messages that target both environmental awareness and social norm/culture at the same time may also provide the greatest levels of return in terms of improving attitudes towards engaging with recycling and is important becasue engagment appears to be the most important category. For example targeting emotive subjects such as children and future generations, which were often referenced at various levels in peoples constructs, and linking those with concerns for the environment to create messages that emphasise the need to sustain resources for future generations. 
However, the impact of social norm/culture does not only affect people's attitudes towards engaging with recycling but also on giving and purchasing refurbished mobile phones (Figure 12). In terms of on giving, Panambunan-Ferse and Breiter (2013) suggested that it could be perceived negatively from a cultural perspective. The results of this study identified that the combination of social norm/culture and on giving was a fairly important consideration for particpants, which suggests it is important that educational messages are sensitive to what is most appropriate. In terms of the purchasing refurbished category, which was ranked third, social norm/culture was the second most important subcategory although it was only at a comparatively mid to low level of importance when considered across all other subcategory-category ratings. This suggests that in targeted messaging, the most value may be gained from targeting the impact of social norm/culture in terms of engagement but not necessarily in terms of on giving or purchasing refurbished.

\section{Age of technology and Perception}

The age of technology subcategory and its relationship with purchasing refurbished phones was the only combination under purchasing refurbished that had a significantly high subcategory-category ranking (Figure 13). This suggests that the age of technology is a significant consideration when people consider purchasing a refurbished mobile phone. This appears to support Ylä-Mella et al. (2015) where is was asserted that the short lifespan of technology is a concern for consumers, which in turn supports suggestions that people are concerned about refurbished mobile phones becoming obsolete (van Weelden et al., 2015). Whilst it is possible to suggest that this concern may only occur in first world countries where consumers are aware of the fast pace of technology and therefore more sensitive to technological changes, it is likely to also be valid in third world countries that have larger second hand markets. That is because the age of technology is a relative concept, in that the perception people have of the age of technology is relative to the type and age of the technology that is available in their country. It is also important to mention that the age of technology and the potential assumption that older technology may be less favored should be avoided. This study suggests that perception of older technology may be one of preference in instances where it meets requirements; and those requirements appear to centre on function and cost.

\section{Spare, Functioning and Residual value}

All three of these subcategories appear under storing and appear to hold a mid-range level of importance in terms of people's attitudes towards storing old mobile phones (Figure 13). However, 
whilst there are concerns around having a spare and whether or not it is functioning, it does not appear these concerns are founded on actual experiences or outcomes. For instance, when it comes to having a spare mobile phone, Huang and Truong (2008) identified that those who store their phones do not often end up using it and Welfens et al. (2013) suggested some old mobile phones are stored even when they are no longer functioning. This suggests it may be more than just experience that is driving the relevance people place on these areas. Perhaps it relates to the need to collect and store resources, which seems more closely aligned with human instinct than learned experience. It is also possible that functioning in some ways is related to having a spare mobile phone for some people and may explain why it has a similar level of importance. However, the link between the two is not clear, and may not exist for everyone, because as previously mentioned, some people store old mobile phones even when they are no longer working.

As with the potential relationship between the spare and functioning subcategories, residual value may be related as well, in that the residual value of a phone may be tied to the fact that it still works. What was clear from this study is that when it comes to residual value the most important aspect overall for participants was the financial residual value. This materialistic form of residual value, as opposed to emotional value, may be easier to manage when trying to discourage people from storing their old mobile phone. This is because the transfer of money, particularly where money is not a major issue, may be easier than trying to manage the transfer of items that hold sentimental value. Overall, whilst these three subcategories are different enough to warrant being categorized separately there does appear to be some potential relationships between them, which suggests when trying to manage them as part of an environmental awareness program, consideration should be given towards targeting them as a group.

\section{Ease}

In this study ease was linked to both the storing of mobile phones and engagement and recycling (Figure 12). In terms of storing, ease has been identifed by several authors (Thavalingam \& Karunasena, 2016; Ylä-Mella et al., 2015) as a likely to impact people's decision to store mobile phones. This study seems to support this assertion, however, the level of importance which participants attributed to ease was only minimal compared to other factors (Figure 13). In terms of engagement, ease received no coded constructs indicating it did not form any part of the participants core beliefs. This may suggest that approaches that promote ease to enourage engagement, such as 
take back schemes in highly accessible places (Silveira \& Chang, 2010), or even permanent instalations (Ylä-Mella et al., 2015) may not actually work as intended. The real contribution of these highly visible and accessible takeback places may be to increase the level of a person's environmental awareness which in turn affects engagement.

\section{Size}

This study appears to suggest, that whilst a mobile phone's size is considered when storing it is not a significant consideration (Figure 13). This appears to support several authors assertions that the small size of old mobile phones does not create the same type of barrier compared to other large electronic devices, and therefore does not inhibit a consumers decisions to store them (Darby \& Obara, 2005; Welfens et al., 2015). The inherent size of a mobile phone may mean its size is unlikey to ever create a barrier to being stored. However, it may be possible to incentivise people to not store them by linking the size of the mobile phone to environmental messages that suggest whilst small the environmental impact can be large.

\section{Trust (Data)}

A study by Baxter and Gram-Hanssen (2016) suggested that concerns around information security did not appear to be a major concern in household surveys and as a result may be more of a concern for businesses. The results of this study may partially support this perpsective because the subcategory category combination of trust(data) and ongiving only had a very low subcategorycategory ranking whilst trust(data) in combination with other categories received the lowest ranking as no constructs were coded to them (Figure 13). This suggests that particpants in general had virtually no concerns around the misuse of data. However, this may not necssarly hold true at the individual level, expecially where a person has experinced a life event that affects the use of their personal information in a untrustworthy way. However, unless educational messages are specifically targeted towards people who have experienced issues with the personal data, messages that attempt to address fears around trust(data) may only have a limited impact since it does not appear to be of great concern from a wider group perspective.

\section{Knowledge, Price and Income}

Knowledge, price, and income were all associated with purchasing refurbished mobile phones and all had a relatively low level of importance (Figure 13). Knowledge and price are both subcategories 
that are used to help judge the benefit versus risks of used mobile phones (van Weelden et al., 2015), whilst income is more aligned to the relative financial status of individuals (Wang et al., 2011). Since it has been suggested that third world countries have a more developed second hand mobile phone market (Thavalingam \& Karunasena, 2016), it is possible to surmise that the second hand mobile phone market in New Zealand is not as developed. This lack of a second hand mobile phone market may be a reflection of people's willingness to purchase. Since there is limited willingness to purchase, the need to place importance on both product knowledge and price is reduced.

In terms of income, its level of importance may also reflect the nature of the demographic group from which the participants came. Thavalingam and Karunasena (2016) suggest that financial status is a barrier to purchasing a mobile phone, however, this may only be the case when there is a significant gap between the price of a mobile phone and their relative income. A person in a third world country earning a few dollars a day is unlikely to be able to afford a mobile phone, whilst a poorer person in a first world country who either receives some government financial support, or who earns a minimum wage, may still be able to afford a mobile phone, albeit a budget one. As a result, whilst there was a significant difference in the income levels of participants in this study, their relative income compared to the potential price of a mobile phone did not appear to be an issue, which may explain why price only had a low level of importance. This relative income effect may also be the reason why income did not receive any coded constructs in terms of its association with engaging with recycling.

\section{Macro-economic}

A new subcategory, macro-economic, was not initially identified and emerged from the coding process of the interviews in respect to engagement with recycling (Appendix 2). Even though it had constructs coded to it, it still had a low ranking compared to other subcategories that also had constructs coded against them, indicating it was not of significant importance at the group level. In addition, consideration was also given to whether or not macroeconomic, and the thought of job creation, was a form of non-monetary incentive and should therefore be included within the incentive factor by expanding the original definition. However, if this were done there would then be an argument to include other factors under engagement such as ease and regulations that have remained separate (Figure 12). Ultimately, it was decided that macroeconomic represented a distinct enough definition within the scope of this study to remain separate. It also adds weight to the idea 
that whilst many factors are constant across the majority of participants, some factors are unique to individuals, which in turn reflects the uniqueness of some of their beliefs.

\section{Incentives and Cost}

In this study the incentives subcategory received no constructs during content analysis (Figure 12) this seems to support Speake and Yangke (2015) who suggested incentives did not appear to be a significant factor when it comes to motivating consumers to engage in take back programmes. If incentives do not form part of a person's core belief system, it seems unlikely that their use as a motivational tool, even if they are in the form of material compensation, as suggested by Welfens et al. (2015) will work. However, this non-significant outcome appears to be contrary to Ongondo and Williams (2011a) who suggested incentives are an important factor that needs to be considered when trying to improve engagement. These contrary positions may indicate that the effect of incentives is not straight forward, and that other factors may be playing a role in determining its overall effectiveness.

It has also been suggested that cost is a factor in terms of encouraging consumers to engage in take back programmes (Huang \& Truong, 2008; Wilhelm et al., 2015). However, the results of this study indicate cost does not form a part of the participants' core beliefs and therefore does not present an area of concern. Whilst this suggests cost is not a barrier, this perspective may only be true initially, and should costs become prohibitive it may affect engagement.

\section{Reputation and experience}

Two subcategories, reputation and experience, did not receive any coded constructs in relation to the category of purchasing refurbished mobile phones, whilst experience was not coded to any constructs in relation to engagement (Figure 12). This suggests reputation and experience, in terms of purchasing a refurbished mobile phone, do not form the basis of any participants' core beliefs. It is possible that their lack of importance, as with other factors related to refurbishing mobile phones, reflects the lack of a secondhand market for used mobile within New Zealand. If the same study were to be conducted in a third world country, where second hand mobile phones make up a greater market share, the significance of reputation and experience may be much greater. Alternatively, it could be suggested that this is a reflection of the potential complex links that exist between brand and decision-making as suggested by Abbey, Meloy, Guide, et al. (2015). However, this seems 
unlikely because both reputation and experience did not have any constructs coded to them, indicting they did not actually exist within the core beliefs of the participants. For the complex links to exist, the factors themselves would need to first exist within the belief system of the participants.

The lack of constructs coded to experience in terms of engagement also appears to suggest that experience/habit does not affect people's attitudes towards engagement. This is in contrast to Speake and Yangke (2015) where it was suggested there was a link between the formation of habits in terms of recycling and Welfens et al. (2015) who suggested the step to recycling e-waste may be easier if habits already exist. If people's core beliefs are developed from their experiences then the lack of core beliefs may suggest recycling, in general, was not yet an ingrained habit within the participants in this study. This suggests there may be a chicken and the egg scenario, where people have developed their core beliefs around recycling have done so because of their experiences, whereas people who have no experience with recycling may not have developed their core beliefs relative to recycling. Based on this assertion and since nobody in the study seemed to express any indepth experience or habits in regards recycling, they would in theory, not have yet developed those core beliefs.

\section{Trade in/Sell, Regulations}

When it comes to the idea of trading in or selling a used mobile phone, the low subcategory and subcategory-category rank (Figure 12) indicate that participants did not consider this option when thinking about managing used mobile phones and may be another symptom of the presence of a limited used mobile phone market. Yin et al. (2014) suggested that consumers might be inhibited from disposing of their mobile phones through recycling schemes if they are able to realize some economic gain from selling them. However, the low level of significance attributed to selling, in combination with the low-level importance of other related factors such as incentives and cost; suggest that the potential to sell a mobile phone is not an inhibiting factor for participants when it comes to sending a used mobile phone for recycling. However, there may be some justification in suggesting that the ability to realize economic gain may be more prevalent in third world countries, where the potential gain from selling a used mobile phone, may be significantly higher especially where relative levels of wealth are low. 
It has also been suggested that the absence of regulations may be linked to a lack of engagement (Yin et al., 2014) and that programmes such as take back schemes should be made mandatory (Silveira \& Chang, 2010). This study identifies that regulations are not a consideration when it comes to peoples core beliefs, however, whilst not important in the context of this group of participants, regulations may form part of consumers core beliefs in countries that are more authoritarian and less liberal in nature. This lack of consideration for regulations in core beliefs does not mean regulations would not work. That is because the influence of regulations is different from other factors, regulations do not rely on voluntary engagement; instead, regulations are compulsory in nature, which means the voluntary component of engagement is removed. Therefore, whilst consumers may not believe in them, they may still respond to them.

\section{Helping}

A significant amount of consideration was given to whether or not the subcategories helping and social norm/culture should be defined as a single subcategory both during subcategory development and during the results analysis. During the development of the intial 3R model it was decided that there was enough of a seperation to justify two different subcategories. One reason was because the decision to help sombody by passing on an old mobile phone, whilst linked a persons societal and cultural background (Hansmann, Bernasconi, Smieszek, Loukopoulos, \& Scholz, 2006), does not neccsiarily rely on a persons social norm/cultural experince. However, in hindsight it was decided the potential effect of peoples experiences may have been underestimated. The first part of the justification for this change can be found during the development of the social norm/culture subcategory where it was suggested that "whilst some users do pass on their old mobile phones because they may want to help, the decision to do so may be affected by social or cultural perceptions". The second part of the justification, and the point that created enough weight to shift to a merger of subcategories, was that the constructs that were coded to helping, fitted just as neatly within social norm/culture. The decision to merge also appeared to be supported when the change was considered in the context of other authors suggestions that users give their old phones to family and friends (Ongondo \& Williams, 2011a; Welfens et al., 2015; Yin et al., 2014), that is because those family and friends form the basis of that persons social and cultural experiences. 


\section{Age and Gender}

Both age and gender were identified in the original model but failed to have any of the participants core constructs coded against them. There are a number of reasons as to why this may have occurred. Since core constructs are viewed as representing what is truly important to an individual in the construct hierarchy it is possible some of the subcategories introduced in the original $3 \mathrm{R}$ model did not actually reflective people's core beliefs. For example, Ongondo and Williams (2011a) suggested there may be a link between gender and mobile phone storage, whilst Darby and Obara (2005) suggest that females are more likely to give an old mobile phone to charity rather than a male. Finally, both Speake and Yangke (2015) and Darby and Obara (2005) suggest that females were more engaged or more willing to recycle compared to males. Whilst these studies indicate gender may have an impact; it does not appear likely to be because a man or a woman strongly identifies with their gender as a reason to store, purchase refurbished or engage with recycling. The same logic applies to demographic data such as age and education level. For example, although Ongondo and Williams (2011a) proposed that older people are more likely to engage in recycling and Yin et al. (2014) identified the impact of education level, no participant in this study suggested they had a deep core belief that was driven by their perception of their age or education level. As a result, it is proposed that this type of demographic information, which is not created from life's experiences, but is more an expression of a person's genetic makeup, has a more indirect influence on people's life experiences and therefore the factors and attitudes that are contained within the 3R model (Figure 14). 


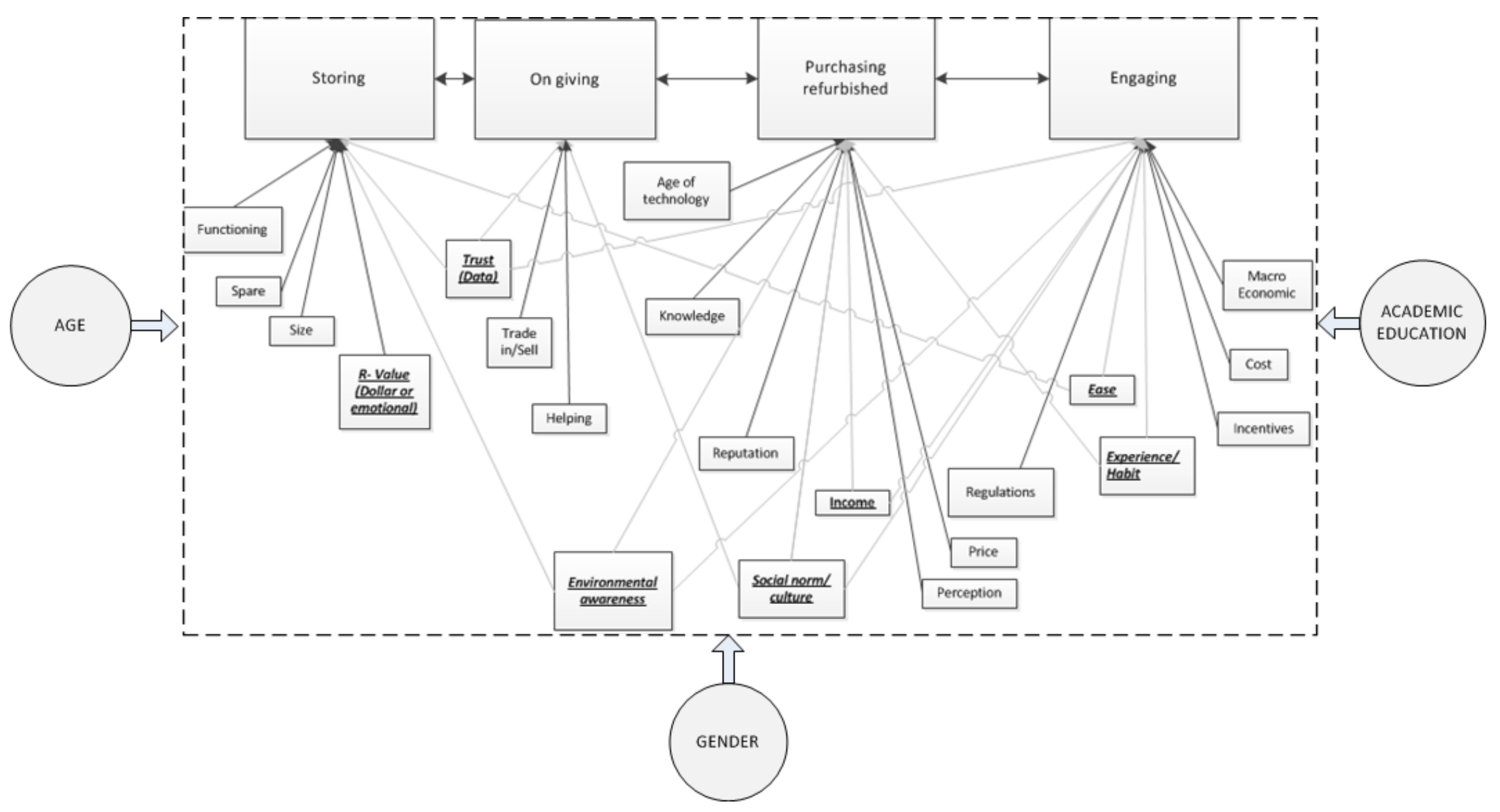

Figure 14. Demographic influences on 3R model 


\section{Conclusion}

The final section of this research will review some of the studies key findings whilst also discussing the implications for both theory and practice and identifying areas for future research.

A number of underlying factors were initially identified as likely to affect consumer attitudes towards the sustainable management of used mobile phones. However, the results of this study suggest not all of those factors appear to have a role to play when it comes to consumers core beliefs, and of those factors that do, there is a broad variety in their level of perceived importance. Environmental awareness was identified as the most important factor in the $3 \mathrm{R}$ model and touches on all attitudes, from reuse to purchasing refurbished mobile phones and recycling. This means that consumers', as a group, have core beliefs that reflect a significant underlying concern for the environment, however, the level of concern at the individual level is varied. Environmental awareness with its strong association with engagement means success in raising environmental awareness will improve attitudes towards engaging, which has in turn been identified as the single most important attitude. Environmental awareness's association with storing is also associated with concern for the environment but this concern, which leads to phones being stored, is actually contrary to an environmentally sustainable approach. This means that educational messaging that works on raising awareness levels should also discourage the storing of old mobile phones by highlighting the negative environment impacts. This educational approach with its focus on environmental awareness may be the single most effective way of improving how users manage their used mobile phones.

This study has also identified that a person's social norms or cultural experiences are a significant factor associated with on giving, purchasing refurbished, and engaging, and only second in importance to environmental awareness. These beliefs are generally linked to concerns around more emotive subjects such as family, children, and future generations. The emotive nature of these beliefs could be leveraged by incorporating them into the same educational messages used to promote environmental awareness. This could be achieved by highlighting how, by not managing old mobile phones in terms of waste production and over use of resources; the negative environmental issues faced by future generations will be significant. 
Another important result of this study is the limited importance that was attributed to both the attitude of purchasing refurbished mobile phones and all but one of the factors that were related to it. This confirms the relative lack of importance second hand mobile phones hold in the belief structure of participants and is a reflection of the idea that non third world countries have less developed second hand phone markets. The one factor that was identified to be important, the age of technology, indicates this is a significant consideration for people when thinking about used mobile phones. However, this area of concern does not necessarily originate from a negative perspective; rather, the age of technology can be considered positive due to its economic value compared to new mobile phones, and where the perceived functionality of the used phone meets requirements.

One final point identified in this study is that peoples' attitudes towards storing mobile phones or engaging in recycling do not seem to be significantly influenced by the perceived ease of undertaking these activities. Therefore, engagement in take back programmes that promote the idea of ease may not be experiencing engagement because participants are concerned about ease, but rather the program message works on their environmental awareness and resonating with core beliefs that reflect concern for the environment. Overall, the results of this study provide a direction in which to target educational messaging on sustainability and the impact of used mobile phones, and these messages should focus on factors identified as important in two of the three R's in the 3R model, reusing and recycling, in order to achieve maximum value.

\subsection{Implications for theory}

This research has taken an existing strategy, originally proposed as a way to manage e-waste sustainably, and applied it to a specific area of concern, used mobile phones. It has drawn on a range of literature from different fields, whilst adopting variants of tried and tested interview and coding techniques in order to both collect and analyze the data. Whilst individually these various aspects are not unique, in combination, they represent an attempt to approach and understand an established problem from a different perspective. This has resulted in a qualitative study that has not only captured the core beliefs of individuals but has synthesised them into a group perspective, resulting in an explanatory model that reflects those core beliefs. This study has shown that in terms of people's core beliefs, reuse and recycling are perceived as closer to ideal when compared to other 
approaches like refurbishing, and it has been established that collectively all of these approaches are seen by consumers as positive ways to manage used mobile phones.

It has collated and presented factors that have been identified across a number of studies as affecting how consumers deal with their used mobile phones, and identified that those factors do not necessarily hold equal importance in terms of their influence across consumers' attitudes. It has identified that factors of a more demographic nature appear to utilize a different pathway of influence in terms of the affect they have on people's attitudes when compared with people's core beliefs and has suggested that more subtle relationships may exist between factors and attitudes, other than the more obvious direct ones. Finally, it has resulted in the understanding that people's core beliefs do reflect a concern for the environment regardless of what actions they ultimately take and has provided support to the idea that mobile phones are stored because of concern for the environment, not necessary because it is the easiest option.

\subsection{Implications for practice}

This study has several implications in terms of practice. Firstly, it allows practitioners to focus on improving sustainability practices by concentrating on the areas of greatest influence in terms of consumer attitudes. It presents an opportunity to create more targeted educational messages that leverage the high importance consumers appear to place on areas such as environmental awareness and social norms and culture while limiting the effort required to be spent on areas that present lesser returns such as promoting the purchasing of refurbished mobile phones. It suggests that practitioners may not need to focus as much on making it easy for consumers to engage with take back programmes, or overcome the ease perceived with storing a used mobile phone because ease is not considered significant by consumers. This study has also identified that when it comes to either engaging with recycling or trading in used mobile phones, factors such as ease, cost, incentives, and income have limited or no concern to consumers, which suggests that they have a level of cost and convenience tolerance when it comes to trading in or recycling used mobile phones.

This means that businesses that offer financial returns on used mobile phones, or are concerned with the need to offset consumers' costs in order to encourage engagement may be focusing too much on the wrong sort of incentives. Alternatively, businesses should focus more energy tapping into the underlying concerns around children and future generations by promoting the 
environmental issues that are associated with the storing of used mobile phones. By leveraging these underlying beliefs not only would there be an increased level of engagement from consumers, it could potentially lead to improved corporate images for mobile phone providers, because of the perception that they are in touch with consumer concerns and actively working to address them.

In New Zealand, the current telecommunication providers' participation in the Telecommunications forum (TCF) is a good indication they see value in voluntary engagement, however, the funding of educational programmes beyond providing current messaging such as take back bins in shops and paid postage for sending in phones it may be an issue. That is because, whilst the New Zealand government supports initiatives that promote the sustainable management of used mobile phones, it does not appear willing to provide funding (Fletcher, 2014). As a result, any required funding would need to be sourced directly from other stakeholders. This may prove problematic, as members of the TCF would need to see a sound return on the type of increased financial investment required especially where a change in the current cost benefit ratio may act as strong disincentive. These increased costs, however, could potentially be offset by the savings made in offering lower returns on used mobile phone trade ins and charging for services such as return postage when phones are being returned for recycling. Additional investment could potentially be sourced from other environmentally concerned parties such as Greenpeace if they were convinced a partnership with industry was able to provide practical solutions.

\subsection{Research Limitations}

This study, as with other types of research, has limitations related to the research approach under taken.This section will disscuss those limitations and there potential implications.

Firstly, there are potential limitations associated with the use of the repgrid interview technique that was adopted for this research. Whilst it was specifically selected because it allowed access to the participants core constructs that might otherwise be inaccessable through other, more standard interview techniques, the technique did present several challenges that may have impacted the interview outcomes. For example, the number of elements used in the repgrids, whilst representing in general the area under investigation, does raise the question as to whether or not they were diverse enough, in terms of their total number, to elicit a truly representative number of constructs. Some participants found it difficult to distinguish between some elements such as reuse and 
refurbish, in that for them, they represented concepts that were either similar or the same. Even where participants did suggest they were different, the majority of the time, they were perceived to be positive ways of dealing with used mobile phones although to varying degrees. It may have been more beneficial to allow the particpants to generate the elements to be used rather than using researcher supplied elements. Whilst this would have resulted in less control, and would have allowed particpants to extend concepts beyond the intended scope of the study as well as create potential difficulty in comparing results across participants, it may have provided a richer more representative source of elements and therefore helped with the elicitation of a broader range of constructs.

The adoption of a convenience sampling approach, whilst appropriate brings with it concerns around how representative the participants are of the general population. Whilst for this study, care was taken to try to ensure there was a diverse representation; it is not possible to determine how representative the participant group was. The fact that all participants were known to the researcher creates a point of commonality from which some degree of under representation could be inferred. It also raises the issue of researcher bias, and the ability to be objective, which whilst inherent when adopting an interpretative approach, may have been further affected by the relationships with participants.

Another potential limitation, also related to the use of a qualitative study, was the approach adopted for validating the coding of repgrid constructs when using Honey's content analysis. As with any coding process, a validity check was undertaken, this for this study consisted of the research supervisor validating the appropriateness of the codes and the coding process. However, this simplified inter coder reliability check only focused on a sample of the codes and did not include the use of additional independent coders or the use of any statistical checks such as Krippendorff's alpha. Although the degree of validity checking adopted for this study was deemed appropriate for the size and scope of study, the limitations of this approach need to be recognized.

Finally, the factors presented in the $3 \mathrm{R}$ model do not appear to be comprehensive and only represent those relative to the participants' core beliefs. As a result, this study did not succeed in explaining the relative importance of other types of factors, those that are more demographic nature, such as age and gender and failed to expose some of the more subtle inter-factor relationships that 
are suggested to exist. This means that whilst this study has generated some thought provoking results it is not exhaustive in its explanatory power, and as a result, the $3 \mathrm{R}$ model needs to undergo further testing and refinement.

\subsection{Future research}

It is suggested that future studies examine the relationships proposed in the $3 \mathrm{R}$ model statistically, on a much larger, more representative sample to determine both the relationships strengths and validity. This would not only help with validating the relationships that have been suggested to exist in the 3R model but also overcome some of the current studies limitations in being able to confidently generalize the results to the wider population.

Future studies could also investigate the more subtle relationships that are suggested to exist between subcategories in this study. In that vein, exploring consumers constructs using a pyramiding technique during repgrids elicitation to build up a visual understanding of their hierarchical constructs would help in linking their core constructs or beliefs with their subordinate constructs. Since lower, subordinate constructs are more specific and linked more closely with a person's behavioral traits this would also provide a bridge to help determine how attitudes towards reuse, refurbishing, and recycling may be expressed behaviorally.

\subsection{Concluding comments}

This study attempted to identify what factors and consumer attitudes affect the sustainable management of used mobile phones, and develop a model that could be used to explain the relationships. A strategy that was discussed by Murugesan (2008) as a way to sustainability manage ewaste, the 3R strategy was used as a lens through which to understand both the factors and consumer attitudes. This research has been successful in identifying a number of underlying factors and the associated consumer attitudes that are affected when considering the sustainable management of used mobile phones. It has identified that some factors such as environmental awareness and social norm/culture appear to have a significant impact on several consumer attitudes whilst having a minimal affect on others. It has strengthened the link between how people's attitudes towards storing mobile phones are affected by environmental awareness and has highlighted how factors such as ease and incentives, whilst currently identified to promote engagement, only appear to be of limited importance to consumers. In summary, this research adds the current body of 
knowledge and may play some small part in changing the way we manage the burden of used mobile phones. 


\section{References}

Abbey, J. D., Meloy, M. G., Blackburn, J., \& Guide, V. D. R. (2015). Consumer Markets for Remanufactured and Refurbished Products. California Management Review, 57(4), 26-42.

Abbey, J. D., Meloy, M. G., Guide, V. D. R., \& Atalay, S. (2015). Remanufactured Products in Closed-Loop Supply Chains for Consumer Goods. Production and Operations Management, 24(3), 488-503.

Amoyaw-Osei, Y., Agyekum, O., Pwamang, J., Mueller, E., Fasko, R., \& Schluep, M. (2011). Ghana e-waste country assessment. SBC e-waste Africa project. Dubendorf, EMPA.

Anderson, C. (2010). Presenting and Evaluating Qualitative Research. American Journal of Pharmaceutical Education, 74(8), 141.

Archer, S. (1988). Qualitative research and the epistemological problems of the management disciplines. In A. Pettigrew (Ed.), Competitiveness and the Management process (pp. 265-302). Oxford: Basil Blackwell.

Barber, N., Taylor, D. C., \& Dodd, T. (2009). The importance of wine bottle closures in retail purchase decisions of consumers. Journal of Hospitality Marketing \& Management, 18(6), 597-614.

Basel Convention. (2008). Guidance document on the environmentally sound management of used and end-of-life mobile phones. Basel Convention Retrieved from http://archive.basel.int/industry/mppi/documents.html.

Basel Convention. (2011). Mobile Phone Partnership Initiative (MPPI). Retrieved from http://www.basel.int/Implementation/TechnicalAssistance/Partnerships/MPPI/Overview/tabid/ 3268/Default.aspx

Baxter, J., \& Gram-Hanssen, I. (2016). Environmental message framing: Enhancing consumer recycling of mobile phones. Resources, Conservation and Recycling, 109, 96-101.

Bernard, T., \& Flitman, A. (2002). Using Repertory Grid Analysis to Gather Qualitative Data for Information Systems Research. Paper presented at the The Australasian Conference on Information Systems (ACIS).

Bliss, J., Monk, M., \& Ogborn, J. (1983). Qualitative data analysis for educational research: A guide to uses of systemic networks. London, England: Croom Helm.

Braun, V., \& Clarke, V. (2006). Using thematic analysis in psychology. Qualitative research in psychology, 3(2), 77-101.

Brewer, K. M., Harwood, M. L., McCann, C. M., Crengle, S. M., \& Worrall, L. E. (2014). The Use of Interpretive Description Within Kaupapa Māori Research. Qualitative health research, 24(9), 1287-1297.

Brown, B. J., Hanson, M. E., Liverman, D. M., \& Merideth Jr, R. W. (1987). Global sustainability: toward definition. Environmental management, 11(6), 713-719.

Caelli, K., Ray, L., \& Mill, J. (2003). 'Clear as mud': toward greater clarity in generic qualitative research. International journal of qualitative methods, 2(2), 1-13.

Chaplin, L., \& Westervelt, S. (2015). Understanding e-Stewards import, export, \& transit requiremets. Retrieved from http://arcadiansolutions.com/wp-content/uploads/Understanding-e-StewardsTrade-Restrictions-2015.pdf

Cidell, J. (2010). Content clouds as exploratory qualitative data analysis. Area, 42(4), 514-523.

Commerce Commision. (2015). Annual Telecommunications Monitoring Report 2014. Retrieved from http://www.comcom.govt.nz/regulated-industries/telecommunications/monitoring-reportsand-studies/monitoring-reports/

Darby, L., \& Obara, L. (2005). Household recycling behaviour and attitudes towards the disposal of small electrical and electronic equipment. Resources, Conservation and Recycling, 44(1), 17-35. 
Department for Business Innovation \& Skills. (2014). Restriction of hazardous substances 9RoHS) regulations 2012 - Government guidance notes for RoHS 2 [Press release]. Retrieved from https://www.gov.uk/government/uploads/system/uploads/attachment_data/file/340865/bis14-1011-RoHS-guidance-notes-july-2014.pdf

Edwards, H. M., McDonald, S., \& Young, S. M. (2009). The repertory grid technique: Its place in empirical software engineering research. Information and Software Technology, 51(4), 785-798.

European Commission. (2011). Directive 2011/65/EU of the European Parliament and of the Council of 8 June 2011 on the restriction of the use of certain hazardous substances in electrical and electronic equipment (recast). Official Journal of the European Union L174 EN, 88-110.

European Commission. (2012a). Directive 2012/19/EU of the European Parliament and of the Council of 4 July 2012 on waste electrical and electronic equipment, WEEE. Official Journal of the European Union, 197, 38-71.

European Commission. (2012b). Directive 2012/19/EU of the European Parliament and of the Council of 4 July 2012 on waste electrical and electronic equipment, WEEE. Official Journal of the European Union L, 197, 38-71.

European Commission. (2016). Waste Electrical \& Electronic Equipment (WEEE). Retrieved from http://ec.europa.eu/environment/waste/weee/index_en.htm

Fallman, D., \& Waterworth, J. (2005). Dealing with user experience and affective evaluation in hci design: A repertory grid approach. Paper presented at the Workshop Paper, $\mathrm{CHI}$.

Fletcher, H. (2014). Phone recycling scheme gets govt backing. NZ Herald. Retrieved from http://www.nzherald.co.nz/business/news/article.cfm?c_id=3\&objectid=11289751

George. (2016). Apple CEO shares plans for the company's future in India. Retrieved from http://www.gsmarena.com/apple_ceo_shares_plans_for_the_company_future_in_india-news18341.php

Geyer, R., \& Blass, V. D. (2010). The economics of cell phone reuse and recycling. The International Journal of Advanced Manufacturing Technology, 47(5-8), 515-525.

Gibbs, S. (2016). Apple only expects your iPhone to last three years. Retrieved from https://www.theguardian.com/technology/2016/apr/15/apple-iphone-last-three-years-maccomputer-four

Glavič, P., \& Lukman, R. (2007). Review of sustainability terms and their definitions. Journal of Cleaner Production, 15(18), 1875-1885.

Grant, K., Goldizen, F. C., Sly, P. D., Brune, M.-N., Neira, M., van den Berg, M., \& Norman, R. E. (2013). Health consequences of exposure to e-waste: a systematic review. The Lancet Global Health, 1(6), 350-361.

Guba, E. G., \& Lincoln, Y. S. (1994). Competing paradigms in qualitative research. In N. K. Denzin \& Y. S. Lincoln (Eds.), Handbook of qualitative research: Sage, Thousand Oaks.

Hansmann, R., Bernasconi, P., Smieszek, T., Loukopoulos, P., \& Scholz, R. W. (2006). Justifications and self-organization as determinants of recycling behavior: The case of used batteries. Resources, Conservation and Recycling, 47(2), 133-159.

HarperCollins. (2016). Collins english dictionary. Retrieved from http://www.collinsdictionary.com/dictionary/english

Hart, S. L. (1997). Beyond greening: strategies for a sustainable world. Harvard Business Review, 75(1), 66-77.

Heacock, M., Kelly, C., Asante, K., Birnbaum, L., Bergman, A., Bruné, M., ... Huo, X. (2015). E-Waste and Harm to Vulnerable Populations: A Growing Global Problem. Environmental health perspectives.

Heacock, M., Kelly, C., \& Suk, W. (2016). E-waste: the growing global problem and next steps. Reviews on environmental health, 31(1), 131-135. 
Hinkle, D. N. (1965). The change of personal constructs from the viewpoint of a theory of construct implications. Ohio State University Columbus.

Hsieh, H.-F., \& Shannon, S. E. (2005). Three approaches to qualitative content analysis. Qualitative health research, 15(9), 1277-1288.

Huang, E. M., \& Truong, K. N. (2008). Breaking the disposable technology paradigm: opportunities for sustainable interaction design for mobile phones. Paper presented at the Proceedings of the SIGCHI Conference on Human Factors in Computing Systems, Florence, Italy.

Hunter, M. G., \& Beck, J. E. (2000). Using repertory grids to conduct cross-cultural information systems research. Information systems research, 11(1), 93-101.

Huawei. (2015). Connecting the Future 2015 Sustainability Report. Retrieved from http://www.huawei.com/en/sustainability/sustainability-report

Jang, Y.-C., \& Kim, M. (2010). Management of used \& end-of-life mobile phones in Korea: a review. Resources, Conservation and Recycling, 55(1), 11-19.

Jankowicz, D. (2005). The easy guide to repertory grids. West Sussex, England: John wiley \& sons.

Kalengayi, F. K. N., Hurtig, A.-K., Ahlm, C., \& Ahlberg, B. M. (2012). "It is a challenge to do it the right way": an interpretive description of caregivers' experiences in caring for migrant patients in Northern Sweden. BMC health services research, 12(1), 433.

Kelly, G. A. (1955). A theory of personal constructs (Vol. 2). New York, USA: W. W. Norton \& Co.

King, A. M., Burgess, S. C., ljomah, W., \& McMahon, C. A. (2006). Reducing waste: repair, recondition, remanufacture or recycle? Sustainable Development, 14(4), 257-267.

Kiron, D., Kruschwitz, N., Haanaes, K., \& Velken, I. V. S. (2012). Sustainability nears a tipping point. MIT Sloan Management Review, 53(2), 69.

Kissling, R., Coughlan, D., Fitzpatrick, C., Boeni, H., Luepschen, C., Andrew, S., \& Dickenson, J. (2013). Success factors and barriers in re-use of electrical and electronic equipment. Resources, Conservation and Recycling, 80, 21-31.

Kondracki, N. L., Wellman, N. S., \& Amundson, D. R. (2002). Content analysis: review of methods and their applications in nutrition education. Journal of nutrition education and behavior, 34(4), 224230.

Landry, M., \& Banville, C. (1992). A disciplined methodological pluralism for MIS research. Accounting, Management and Information Technologies, 2(2), 77-97.

Lasiuk, G. C., Comeau, T., \& Newburn-Cook, C. (2013). Unexpected: an interpretive description of parental traumas' associated with preterm birth. BMC pregnancy and childbirth, 13(1), 1.

Leiserowitz, A. A., Kates, R. W., \& Parris, T. M. (2006). Sustainability values, attitudes, and behaviors: A review of multinational and global trends. Annu. Rev. Environ. Resour., 31, 413-444.

MacGibbon, J., \& Zwimpfer, L. (2006). e-Waste in New Zealand - TAKING RESPONSIBILITY FOR END-OFLIFE COMPUTERS AND TVS Retrieved from http://www.canz.org.nz/Ewaste\%20in\%20NZ,\%20CANZ\%20report,\%20July\%202006\%20-\%20Web\%20version.pdf

Maheu, C., \& Thorne, S. (2008). Receiving inconclusive genetic test results: an interpretive description of the BRCA1/2 experience. Research in nursing \& health, 31(6), 553-562.

Marsden, D., \& Littler, D. (2000). Repertory grid technique-An interpretive research framework. European Journal of Marketing, 34(7), 816-834.

McNaught, C., \& Lam, P. (2010). Using Wordle as a supplementary research tool. The qualitative report, 15(3), 630.

Meister, J. C., \& Willyerd, K. (2010). Mentoring millennials. Harvard Business Review, 88(5), 68-72.

Miles, M. B., Huberman, A. M., \& Saldana, J. (2013). Qualitative data analysis: A methods sourcebook: SAGE Publications, Incorporated. 
Ministry for the Environment. (2007). Basel Convention on the Control of Transboundary Movements of Hazardous Wastes and their Disposal. Retrieved from http://www.mfe.govt.nz/more/international-environmental-agreements/multilateralenvironmental-agreements/key-multilateral-0

Ministry for the Environment. (2014). RE:MOBILE. Retrieved from http://www.mfe.govt.nz/waste/product-stewardship/accredited-voluntary-schemes/remobile

Ministry for the Environment. (2015a). Mandatory product stewardship. Retrieved from http://www.mfe.govt.nz/node/15017/

Ministry for the Environment. (2015b). Waste Minimisation Act 2008.

Ministry of Business Innovation \& Employment. (2015). Regional Economic Activity Report. Retrieved from http://www.mbie.govt.nz/info-services/business/business-growthagenda/regions/documents-image-library/rear-2015/min-a003-rear-report-Ir-optimised.pdf

Morton, J. (2016). Use an old cellphone - save the world. New Zealand Herald. Retrieved from http://www.nzherald.co.nz/technology/news/article.cfm?c_id=5\&objectid=11688090

Murugesan, S. (2008). Harnessing green IT: Principles and practices. IT professional, 10(1), 24-33.

Muscat, A. C. (2010). Elite athletes' experiences of identity changes during a career-ending injury: an interpretive description. (PhD), University of British Columbia. Retrieved from https://open.library.ubc.ca/clRcle/collections/ubctheses/24/items/1.0054291

Myers, M. D. (2013). Qualitative research in business and management: Sage.

Myers, M. D., \& Avison, D. (2002). Qualitative research in information systems: a reader. London, England: Sage.

Neergaard, M. A., Olesen, F., Andersen, R. S., \& Sondergaard, J. (2009). Qualitative description-the poor cousin of health research? BMC medical research methodology, 9(1), 1.

New Zealand Telecommunications Forum. (2017). About RE:MOBILE. Retrieved from http://www.tcf.org.nz/consumers/mobile/mobile-phone-recycling/

Nidumolu, R., Prahalad, C. K., \& Rangaswami, M. R. (2009). Why sustainability is now the key driver of innovation. Harvard Business Review, 87(9), 56-64.

Nnorom, I., Ohakwe, J., \& Osibanjo, O. (2009). Survey of willingness of residents to participate in electronic waste recycling in Nigeria-A case study of mobile phone recycling. Journal of Cleaner Production, 17(18), 1629-1637.

Nnorom, I., \& Osibanjo, O. (2009). Toxicity characterization of waste mobile phone plastics. Journal of hazardous materials, 161(1), 183-188.

Ojeda-Benítez, S., Armijo-de Vega, C., \& Marquez-Montenegro, M. Y. (2008). Household solid waste characterization by family socioeconomic profile as unit of analysis. Resources, Conservation and Recycling, 52(7), 992-999.

Ongondo, F., \& Williams, I. (2011a). Greening academia: Use and disposal of mobile phones among university students. Waste Management, 31(7), 1617-1634.

Ongondo, F., \& Williams, I. (2011b). Mobile phone collection, reuse and recycling in the UK. Waste Management, 31(6), 1307-1315.

Panambunan-Ferse, M., \& Breiter, A. (2013). Assessing the side-effects of ICT development: E-waste production and management: A case study about cell phone end-of-life in Manado, Indonesia. Technology in Society, 35(3), 223-231.

Paszkowska-Rogacz, A., \& Kabzinska, Z. (2012). Applications of Kelly's Personal Construct Theory to Vocational Guidance. Psychology Research, 2(7), 408.

Perkins, D. N., Brune Drisse, M.-N., Nxele, T., \& Sly, P. D. (2014). E-Waste: A Global Hazard. Annals of Global Health, 80(4), 286-295. doi:http://dx.doi.org/10.1016/j.aogh.2014.10.001

Phythian, G. J., \& King, M. (1992). Developing an expert support system for tender enquiry evaluation: a case study. European Journal of Operational Research, 56(1), 15-29. 
Pope, M., \& Denicolo, P. (1993). The art and science of constructivist research in teacher thinking. Teaching and Teacher Education, 9(5), 529-544.

Quariguasi Frota Neto, J., \& Bloemhof-Ruwaard, J. M. (2009). The environmental gains of remanufacturing: evidence from the computer and mobile industry. ERIM Report Series Reference No. ERS-2009-024-LIS.

Rathore, P., Kota, S., \& Chakrabarti, A. (2011). Sustainability through remanufacturing in India: a case study on mobile handsets. Journal of Cleaner Production, 19(15), 1709-1722.

Rivera, J., \& van der Meulen, R. (2014). Gartner says annual smartphone sales surpassed sales of feature phones for the first time in 2013. Retrieved from http://www.gartner.com/newsroom/id/2665715

Russo, M. V., \& Fouts, P. A. (1997). A Resource-Based Perspective on Corporate Environmental Performance and Profitability. The Academy of Management Journal, 40(3), 534-559. doi:10.2307/257052

Saldaña, J. (2013). The coding manual for qualitative researchers. London, England: Sage.

Sandelowski, M. (2000). Focus on research methods-whatever happened to qualitative description? Research in nursing and health, 23(4), 334-340.

Sarath, P., Bonda, S., Mohanty, S., \& Nayak, S. K. (2015). Mobile phone waste management and recycling: Views and trends. Waste Management, 46, 536-545.

Silveira, G. T., \& Chang, S.-Y. (2010). Cell phone recycling experiences in the United States and potential recycling options in Brazil. Waste Management, 30(11), 2278-2291.

Speake, J., \& Yangke, L. N. (2015). "What do I do with my old mobile phones? I just put them in a drawer": Attitudes and perspectives towards the disposal of mobile phones in Liverpool, UK. Human Geographies--Journal of Studies \& Research in Human Geography, 9(2).

STeP. (2013). Annual Report 2012/13. Retrieved from http://www.stepinitiative.org/files/step/StEP_AR/StEP_AR.html

STeP. (2016). What is e-waste. Retrieved from http://www.step-initiative.org/what-is-ewaste.html

Suckling, J., \& Lee, J. (2015). Redefining scope: the true environmental impact of smartphones? The International Journal of Life Cycle Assessment, 20(8), 1181-1196.

Sugiyama, K., Honma, O., \& Mishima, N. (2016). Quantitative Analysis of Material Flow of Used Mobile Phones in Japan. Procedia CIRP, 40, 79-84.

Tan, F. B., \& Hunter, M. G. (2002). The repertory grid technique: A method for the study of cognition in information systems. MIS quarterly, 39-57.

Thavalingam, V., \& Karunasena, G. (2016). Mobile phone waste management in developing countries: A case of Sri Lanka. Resources, Conservation and Recycling, 109, 34-43. doi:http://dx.doi.org/10.1016/j.resconrec.2016.01.017

Thorne, S. (2008). Interpretive description (Vol. 2). New York, USA: Left Coast Press.

Thorne, S., Kirkham, S., \& O'Flynn-Magee, K. (2004). The analytic challenge in interpretive description. International journal of qualitative methods, 3(1), 1-11.

Thorne, S., Kirkham, S. R., \& MacDonald-Emes, J. (1997). Focus on qualitative methods. Interpretive description: a noncategorical qualitative alternative for developing nursing knowledge. Research in nursing \& health, 20(2), 169-177.

Thorne, S., Oliffe, J., KIM-SING, C., Hislop, T., Stajduhar, K., Harris, S., ... Oglov, V. (2010). Helpful communications during the diagnostic period: an interpretive description of patient preferences. European journal of cancer care, 19(6), 746-754.

UL. (2017). Understanding standards. Retrieved from http://ulstandards.ul.com/standard/?id=110_1

United States Enviromental Protection Agency. (2012). Recycling. Retrieved from https://www3.epa.gov/climatechange/wycd/waste/downloads/Recycling.pdf 
van Weelden, E., Mugge, R., \& Bakker, C. (2015). Paving the way towards circular consumption: exploring consumer acceptance of refurbished mobile phones in the Dutch market. Journal of Cleaner Production, 113, 743-754.

Walsham, G. (1995a). The emergence of interpretivism in IS research. Information systems research, 6(4), 376-394.

Walsham, G. (1995b). Interpretive case studies in IS research: nature and method. European Journal of information systems, 4(2), 74-81.

Wang, Z., Zhang, B., Yin, J., \& Zhang, X. (2011). Willingness and behavior towards e-waste recycling for residents in Beijing city, China. Journal of Cleaner Production, 19(9), 977-984.

Welfens, M. J., Nordmann, J., \& Seibt, A. (2015). Drivers and barriers to return and recycling of mobile phones. Case studies of communication and collection campaigns. Journal of Cleaner Production.

Welfens, M. J., Nordmann, J., Seibt, A., \& Schmitt, M. (2013). Acceptance of mobile phone return programmes for increased resource efficiency by young people-experiences from a German research project. Resources, 2(3), 385-405.

Whyte, G., \& Bytheway, A. (1996). Factors affecting information systems' success. International journal of service industry management, 7(1), 74-93.

Wilhelm, M., Hutchins, M., Mars, C., \& Benoit-Norris, C. (2015). An overview of social impacts and their corresponding improvement implications: a mobile phone case study. Journal of Cleaner Production, 102, 302-315.

Wilhelm, W., Yankov, A., \& Magee, P. (2011). Mobile phone consumption behavior and the need for sustainability innovations. Journal of Strategic Innovation and Sustainability, 7(2), 20-40.

Wilson, A. C. (2010). Telecommunications - Mobile phones. Retrieved from http://www.teara.govt.nz/en/telecommunications/page-7

Winter, D. A. (2003). Repertory grid technique as a psychotherapy research measure. Psychotherapy Research, 13(1), 25-42.

Wright, R. P. (2006). Rigor and Relevance Using Repertory Grid Technique in Strategy Research. In D. J. Ketchen, D. J. Ketchen Jr, \& D. D. Bergh (Eds.), Research Methodology in Strategy and Management (Vol. 3, pp. 289-341): Emerald Group Publishing Limited.

Yau, Y. (2012). Stakeholder engagement in waste recycling in a high-rise setting. Sustainable Development, 20(2), 115-127.

Yin, J., Gao, Y., \& Xu, H. (2014). Survey and analysis of consumers' behaviour of waste mobile phone recycling in China. Journal of Cleaner Production, 65, 517-525.

Ylä-Mella, J., Keiski, R. L., \& Pongrácz, E. (2015). Electronic waste recovery in Finland: Consumers' perceptions towards recycling and re-use of mobile phones. Waste Management, 45, 374-384.

Zhao, H.-h., Gao, Q., Wu, Y.-p., Wang, Y., \& Zhu, X.-d. (2014). What affects green consumer behavior in China? A case study from Qingdao. Journal of Cleaner Production, 63, 143-151. 


\section{Appendix}

1) Literature review category matrix and source articles

\begin{tabular}{|c|c|c|c|c|}
\hline $\begin{array}{l}\text { Theme } \\
\text { Nowownot }\end{array}$ & $\begin{array}{l}\text { Category } \\
\text { A Ambuedel }\end{array}$ & 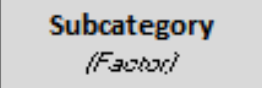 & Example code & Articles \\
\hline & \multirow{9}{*}{ Storage } & Gender & $\begin{array}{l}\text { “...More females than males hoarded } 1 \text { or } 2 \\
\text { mobile phones. How ever, the opposite was } \\
\text { true when considering } 3,5 \text { and } 5+\text { phones; } \\
\text { more males than females hoarded these } \\
\text { number of phones. In total, male students } \\
\text { stockpiled slightly more phones than } \\
\text { females...." }\end{array}$ & (Ongondo \& Williams, 2011a) \\
\hline & & \multirow[b]{2}{*}{ Spare phone } & $\begin{array}{l}\text { "....most respondents claim to keep phones } \\
\text { as spares...." }\end{array}$ & \multirow{2}{*}{ 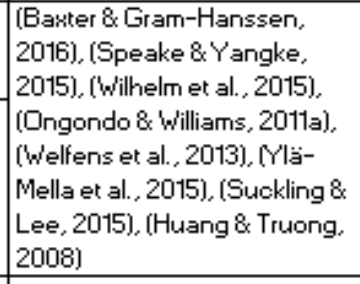 } \\
\hline & & & $\begin{array}{l}\text { "...the most common reason was to keep } \\
\text { them as a spare phone. }\end{array}$ & \\
\hline & & \multirow{2}{*}{ Functioning / broken } & $\begin{array}{l}\text { ¿...people reported keeping phones } \\
\text { knowing they could not use them as } \\
\text { backup because they were broken or not..." }\end{array}$ & \multirow{2}{*}{$\begin{array}{l}\text { (Whelfens et al., 2013), (Huang } \\
\text { \& Truong, 2008), } \\
\text { (Panambunan-Ferse \& Breiter. } \\
\text { 2013) }\end{array}$} \\
\hline & & & $\begin{array}{l}\text { "...not only keep their old mobile phones } \\
\text { that are still working but also their broken } \\
\text { ones." }\end{array}$ & \\
\hline & & \multirow{2}{*}{$\begin{array}{c}\text { Residual value } \\
\text { (Dollax or emotional) }\end{array}$} & $\begin{array}{l}\text { "....consumers seem relatively unwilling to } \\
\text { simply give aw ay this value..." }\end{array}$ & \multirow{2}{*}{$\begin{array}{l}\text { (Ongondo \& Williams, 2011a), } \\
\text { (Thavalingam \& Karunasena, } \\
\text { 2016). (Welfens et al., 2015), } \\
\text { (Bater \& Gram-Hanssen, } \\
\text { 2016). (Ylä-Mella et al. , 2015) }\end{array}$} \\
\hline & & & “... second hand selling price..." & \\
\hline & & \multirow[b]{2}{*}{ Ease of storage } & $\begin{array}{l}\text { “...end of use mobile phones lay at home } \\
\text { because they have not gotten around to } \\
\text { returning it yet." }\end{array}$ & \multirow[b]{2}{*}{$\begin{array}{l}\text { (Thavalingam \& Karunasena, } \\
\text { 2016). (Ylä-Mella et al. 2015) }\end{array}$} \\
\hline & & & $\begin{array}{l}\text { “...high storingrate at homes indicates that } \\
\text { proximity and convenience of the current } \\
\text { system is inadequate to motivate the return } \\
\text { of small wEEE.” }\end{array}$ & \\
\hline
\end{tabular}




\begin{tabular}{|c|c|c|c|c|}
\hline \multirow[t]{7}{*}{ Reuse } & & Size & $\begin{array}{l}\text { "For small electronics and particularly } \\
\text { mobile phones, there are no such physical } \\
\text { barriers....." } \\
\text { "Finally, mobile phones are very small, } \\
\text { unlike bulky refrigerators, for instance. } \\
\text { Hence, owners of old devices do not mind } \\
\text { keeping several generations of mobile } \\
\text { phones at home." }\end{array}$ & $\begin{array}{l}\text { (Welfens et al. , 2015). (Baxter } \\
\text { \& Gram-Hanssen, 2016). } \\
\text { (Darby \& Obara, 2005) }\end{array}$ \\
\hline & & $\begin{array}{c}\text { Education } \\
\text { (Environmental) }\end{array}$ & $\begin{array}{l}\text { "....for mobile phones there is a very } \\
\text { significant third option of do-nothing, } \\
\text { meaning retaining the electronic product } \\
\text { after use." } \\
\text { "...consumers appear relatively aw are of the } \\
\text { issues surrounding electronics and mobile } \\
\text { phone recycling-and yet fail to recycle } \\
\text { mobile phones nearly as extensively as } \\
\text { many other electronic products. The do- } \\
\text { nothing option is extremely attractive to }\end{array}$ & $\begin{array}{l}\text { (Baster \& Gram-Hanssen, } \\
\text { 2016) } \\
\end{array}$ \\
\hline & & Trust (Data) & $\begin{array}{l}\text { "People are afraid that others could enrich } \\
\text { themselves through one's own mobile } \\
\text { phone or misuse personal data. Therefore, } \\
\text { they prefer to keep their old mobile phone." }\end{array}$ & (Welfens et al. , 2015) \\
\hline & \multirow{4}{*}{$\begin{array}{c}\text { Passing to } 3 \text { rd } \\
\text { party }\end{array}$} & Helping/Gifts & $\begin{array}{l}\text { "...passed the phones to other people as } \\
\text { gifts." } \\
\text { "...students passing on phones to someone } \\
\text { else." }\end{array}$ & $\begin{array}{l}\text { (Dngondo \& Williams, 2011a), } \\
\text { (Yin et al. , 2014), ('Welfenset } \\
\text { al. , 2015) }\end{array}$ \\
\hline & & Trade in/Sell & $\begin{array}{l}\text { "...some of them said they will sell their old } \\
\text { mobile phone...or give to it friends." } \\
\text { "...w ould either keep them for personal use } \\
\text { or sell them..." }\end{array}$ & $\begin{array}{l}\text { ('Dngondo \& Williams, 2011a), } \\
\text { (Huang \& Truong, 2008), } \\
\text { ('Welfens et al. , 2013), (Yin et } \\
\text { al., 2014) }\end{array}$ \\
\hline & & Social norm/Culture & $\begin{array}{l}\text { “...users are reluctant to pass their phones } \\
\text { to friends or family, as owning or giving aw ay } \\
\text { an old cell phone is frowned upon." }\end{array}$ & $\begin{array}{l}\text { (Panambunan-Ferse \& Breiter } \\
\text { 2013) }\end{array}$ \\
\hline & & Trust (Data) & $\begin{array}{l}\text { "People are afraid that others could enrich } \\
\text { themselves through one's own mobile } \\
\text { phone or misuse personal data. Therefore, } \\
\text { they prefer to keep their old mobile phone." }\end{array}$ & (Welfens et al. , 2015). \\
\hline
\end{tabular}




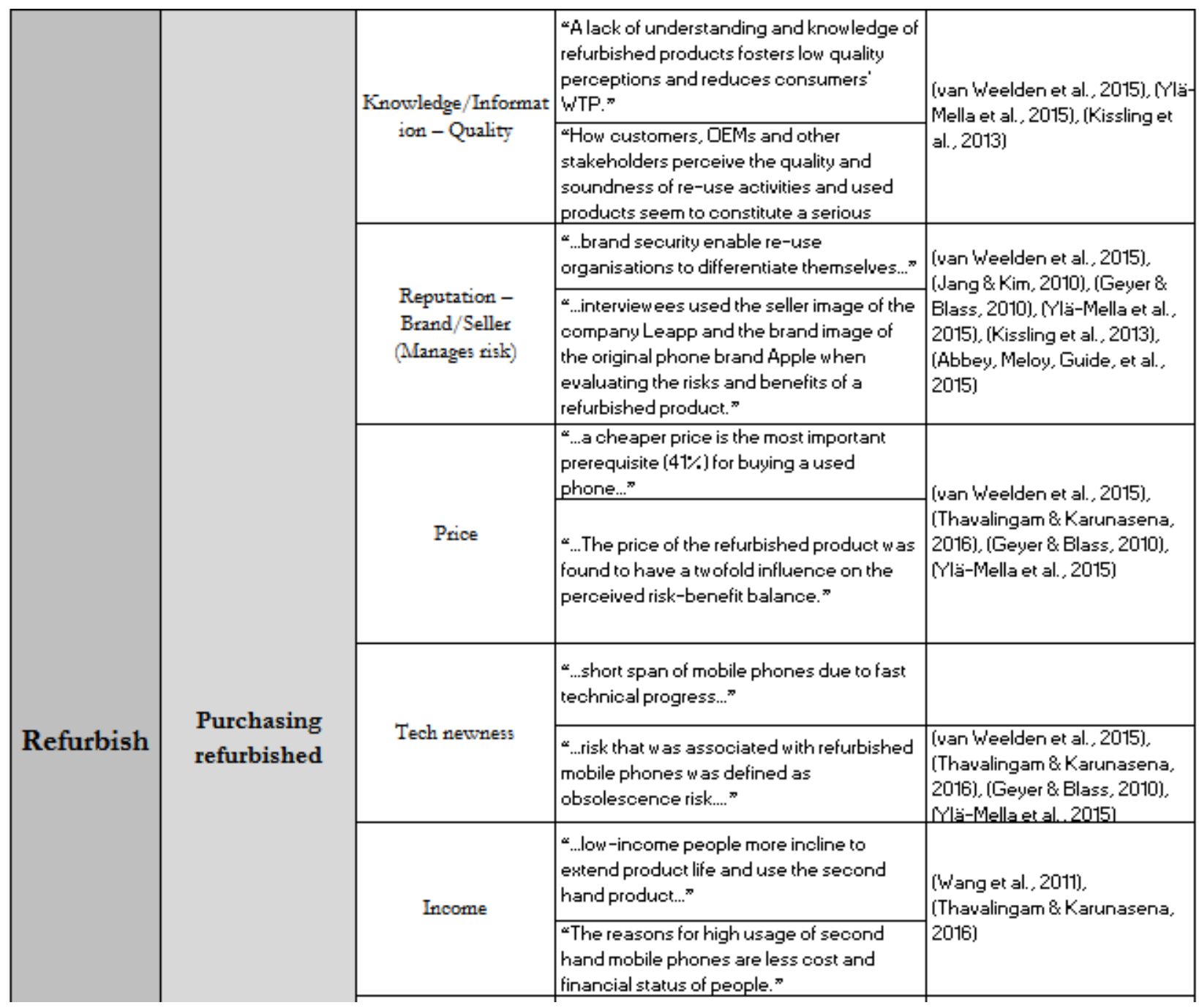




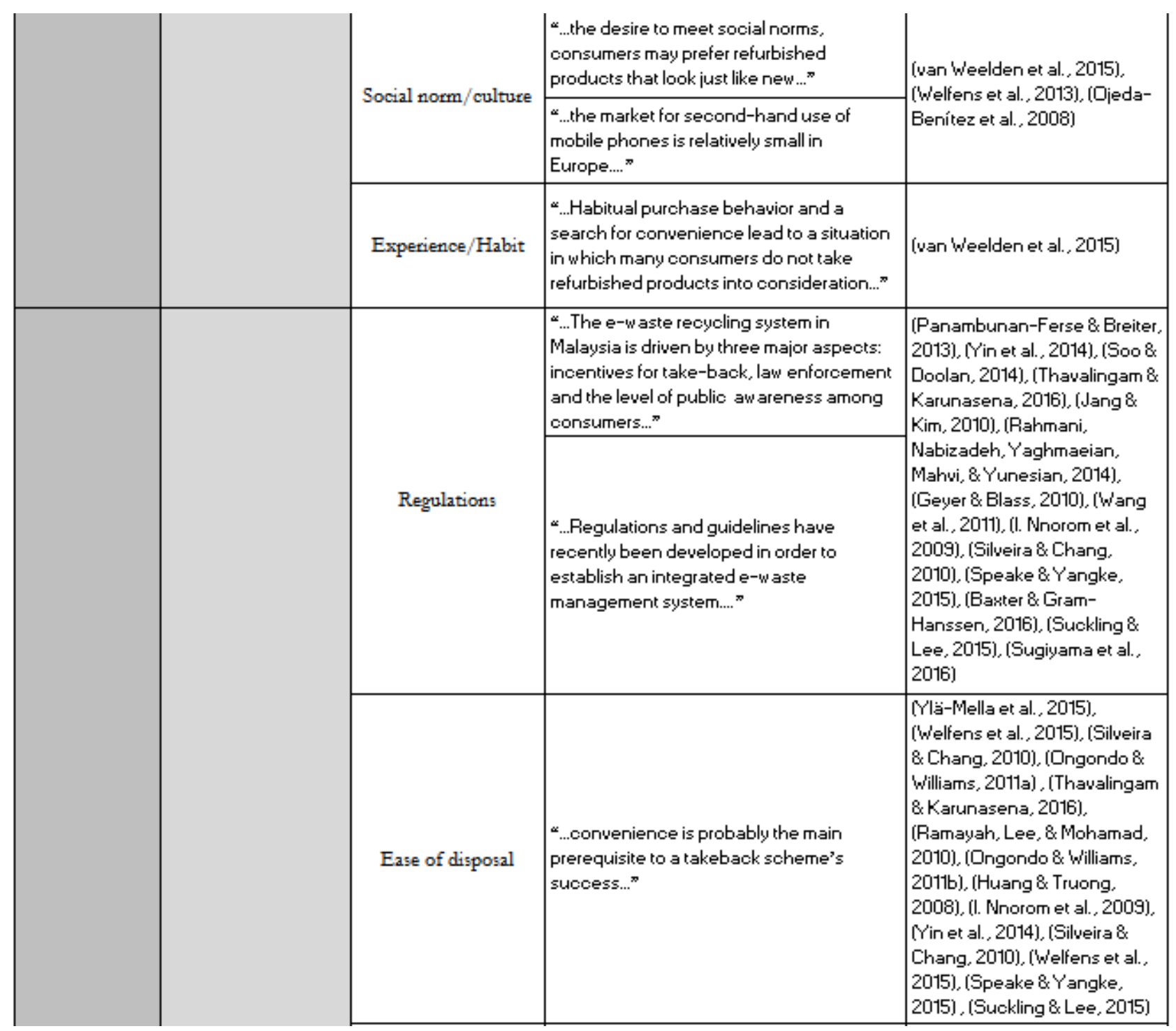




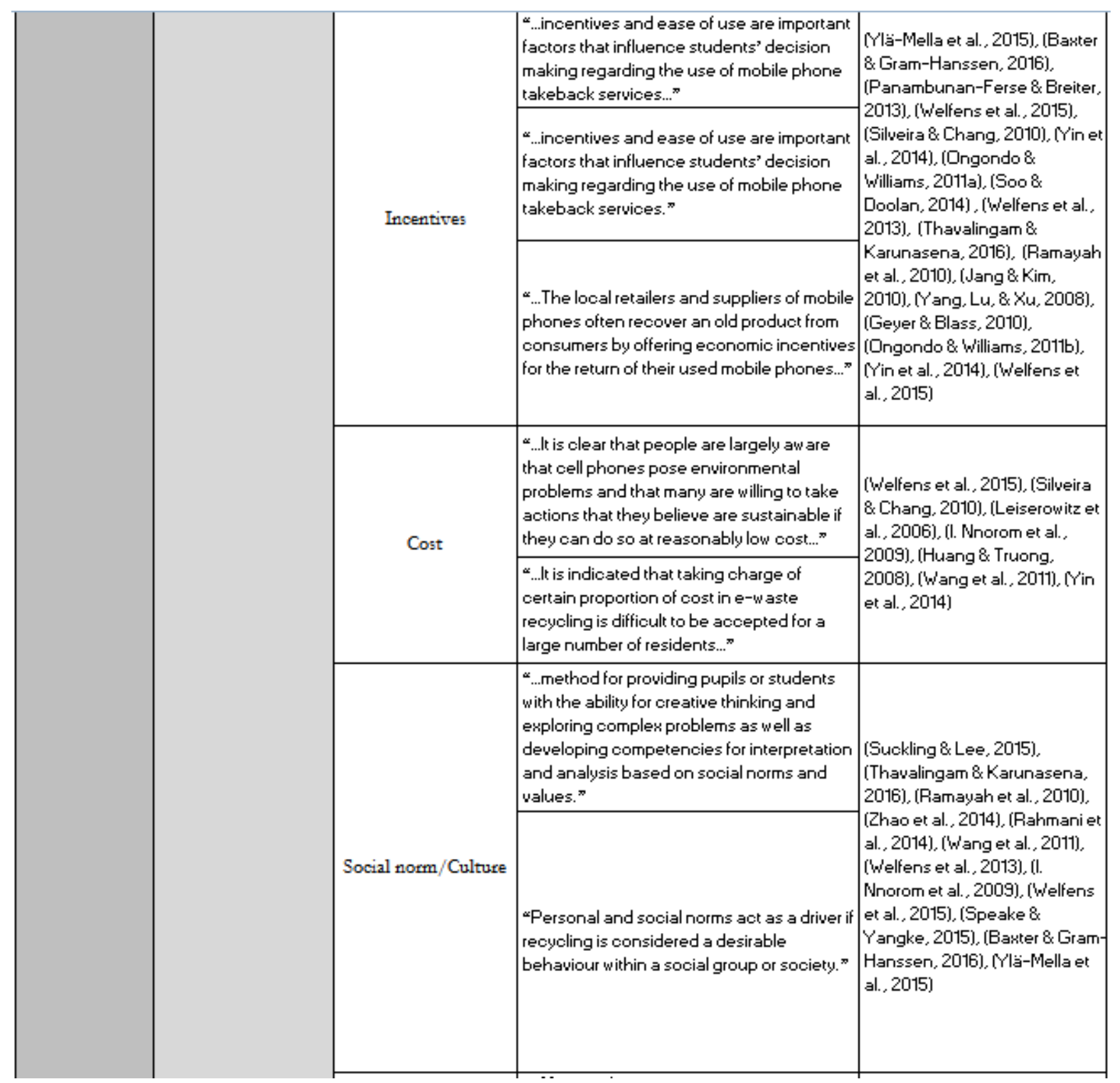




\begin{tabular}{|c|c|c|c|c|}
\hline \multirow[t]{9}{*}{ Recycle } & \multirow[t]{9}{*}{ Engagement } & Trust (Data/General) & $\begin{array}{l}\text { “...Mistrust due to non-transparent } \\
\text { recycling pathw ays is another factor that } \\
\text { influences the return and recycling of } \\
\text { manilenhones } \approx \\
\text { “...lssues relating to data security are often } \\
\text { mentioned in mobile phone and IT } \\
\text { equipment recycling..." }\end{array}$ & $\begin{array}{l}\text { ('Welfens et al., 2013), ('Welfens } \\
\text { et al., 2015), (Baxter \& Gram- } \\
\text { Hanssen, 2016) }\end{array}$ \\
\hline & & \multirow[b]{2}{*}{ Income } & $\begin{array}{l}\text { "...low-income people rarely participate in } \\
\text { the recycling..." }\end{array}$ & \multirow{2}{*}{$\begin{array}{l}\text { (Darby \& Obara, 2005), (Yang } \\
\text { et al., 2008), ('Wang et al., } \\
\text { 2011), (Ramayahet al., 2010), (l. } \\
\text { Nnorom et al., 2009), (Yin et al. } \\
\text { 2014), ('Welfens et al., 2015). } \\
\text { (Zhao et al. , 2014) }\end{array}$} \\
\hline & & & $\begin{array}{l}\text { “...monthly income and education level } \\
\text { have a positive relationship with the } \\
\text { willingness of customers in waste mobile } \\
\text { phone recycling..." }\end{array}$ & \\
\hline & & \multirow[t]{2}{*}{ Gender } & $\begin{array}{l}\text { “...females were also more willing to engage } \\
\text { in mobile phone recycling than males...." }\end{array}$ & \multirow{2}{*}{$\begin{array}{l}\text { (Speake \& Yangke, 2015), } \\
\text { (Darby \& Obara, 2005) }\end{array}$} \\
\hline & & & “...women play a greater role in recycling..." & \\
\hline & & \multirow{2}{*}{ Age } & $\begin{array}{l}\text { “...The relationship between age and green } \\
\text { consumer behavior shows that older } \\
\text { consumers are more likely to engage in } \\
\text { using and recycling behaviors..." }\end{array}$ & \multirow{2}{*}{$\begin{array}{l}\text { (Zhao et al., 2014), (Speake \& } \\
\text { Yangke, 2015), ('Wanget al., } \\
\text { 2011), ('Dngondo \& Williams, } \\
\text { 2011a) }\end{array}$} \\
\hline & & & $\begin{array}{l}\text { “...For age, the results revealed that the age } \\
\text { group } 25-29 \text { years were more willing to } \\
\text { participate in mobile phone recycling than } \\
\text { any other..." }\end{array}$ & \\
\hline & & \multirow[b]{2}{*}{$\begin{array}{l}\text { Education } \\
\text { (Academic) }\end{array}$} & $\begin{array}{l}\text { “...monthly income and education level } \\
\text { have a positive relationship with the } \\
\text { willingness of customers in waste mobile } \\
\text { phone recycling..." }\end{array}$ & \multirow[b]{2}{*}{$\begin{array}{l}\text { (Yin et al., 2014). (Ramayahet } \\
\text { al., 2010), (Ylä-Mella et al., } \\
\text { 2015). (Zhao et al., 2014). } \\
\text { ('Wang et al., 2011) } \\
\end{array}$} \\
\hline & & & $\begin{array}{l}\text { “...better-educated tend to score higher on } \\
\text { all components of the environmental } \\
\text { domain, probably reflecting the fact that } \\
\text { "the very nature of ecology with its complex } \\
\text { interactions between organisms and } \\
\text { environment serves to make its subject } \\
\text { matter difficult to understand and } \\
\text { assimilate"..." }\end{array}$ & \\
\hline
\end{tabular}




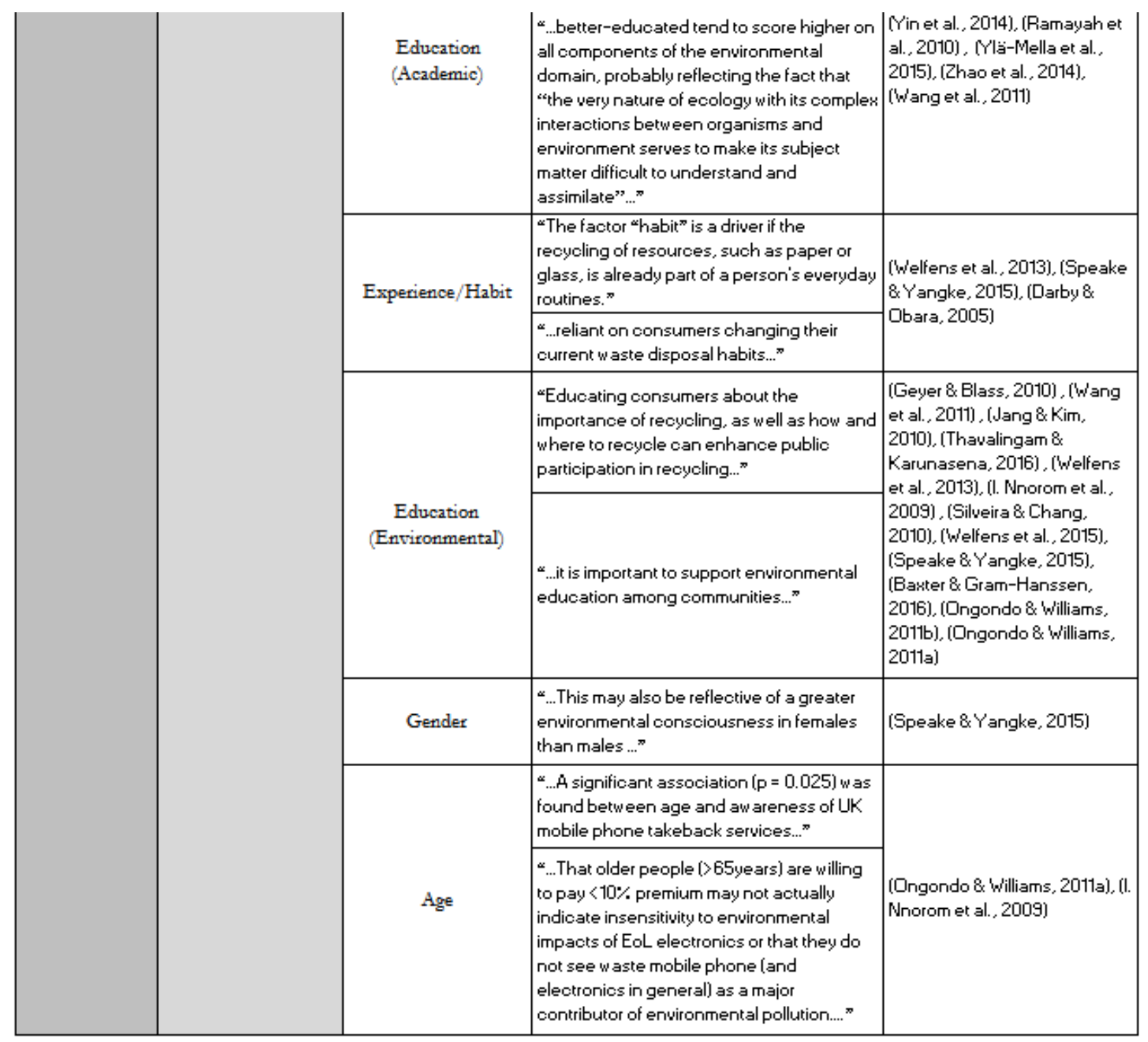


2) Honey content analysis

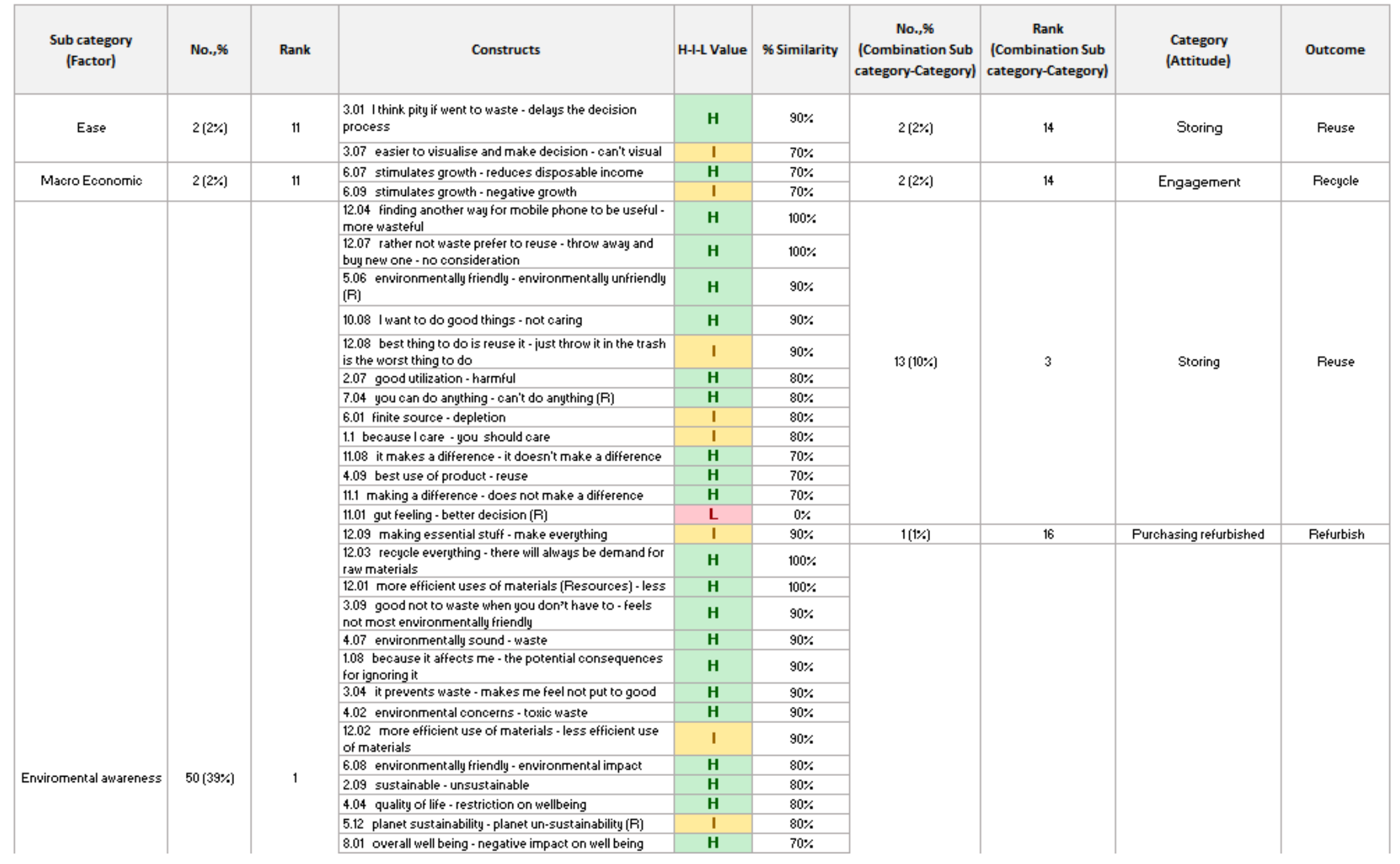




\begin{tabular}{|c|c|c|c|c|c|c|c|c|c|}
\hline & & & 8.1 gives ability to have positive effect - having no effect & H & $70 \%$ & \multirow{22}{*}{$36(28 \%)$} & \multirow{22}{*}{1} & \multirow{22}{*}{ Engagement } & \multirow{22}{*}{ Recycle } \\
\hline & & & 11.04 life is important to me - everything must die & $\mathbf{H}$ & $70 \%$ & & & & \\
\hline & & & 4.06 creates less rubbish - waste of technology & H & $70 \%$ & & & & \\
\hline & & & $\begin{array}{l}3.05 \text { reusing materials - feels bad when something can be } \\
\text { done about it }\end{array}$ & 1 & $70 \%$ & & & & \\
\hline & & & 1.04 the future (our life) - enjoyment (life should be fun) & $\mathbf{I}$ & $70 \%$ & & & & \\
\hline & & & $\begin{array}{l}3.03 \text { good to purchase and recycle - have to make a } \\
\text { decision on impact }\end{array}$ & $\mathbf{I}$ & $70 \%$ & & & & \\
\hline & & & 11.05 acknowledgement of self - self wouldn't make a & I & $60 \%$ & & & & \\
\hline & & & 4.08 best use of product - recycle & I & $60 \%$ & & & & \\
\hline & & & 2.06 sustainable - dangerous & $\mathbf{I}$ & $60 \%$ & & & & \\
\hline & & & 9.09 not using resources - using new & $\mathbf{I}$ & $60 \%$ & & & & \\
\hline & & & $\begin{array}{l}11.07 \text { importance of identification - identifying would not } \\
\text { matter }\end{array}$ & $\mathbf{I}$ & $60 \%$ & & & & \\
\hline & & & 5.01 reducing waste - unsustainable resource use & $\mathbf{L}$ & $60 \%$ & & & & \\
\hline & & & 5.02 making the most of resources - over use of & $\mathbf{L}$ & $60 \%$ & & & & \\
\hline & & & 1.09 we have to look after environment - it's a working & $\mathbf{L}$ & $60 \%$ & & & & \\
\hline & & & 4.05 recycle - rubbish pile up & $\mathbf{I}$ & $50 \%$ & & & & \\
\hline & & & 11.06 to make a difference - to stay with the known & $\mathbf{I}$ & $50 \%$ & & & & \\
\hline & & & 6.02 extinction (Materials) - innovation (R) & $\mathbf{L}$ & $50 \%$ & & & & \\
\hline & & & 8.07 affects your life - has no impact on life & $\mathbf{L}$ & $50 \%$ & & & & \\
\hline & & & $\begin{array}{l}4.03 \text { quality of everyday living - healthy environment } \\
\text { (recycling) (0) }\end{array}$ & $\mathbf{L}$ & $20 \%$ & & & & \\
\hline & & & 2.03 sensible - altruistic & $\mathbf{L}$ & $10 \%$ & & & & \\
\hline & & & 3.08 benefit from a working phone - good for the & $\mathbf{L}$ & $10 \%$ & & & & \\
\hline & & & 9.05 finite resources - unlimited resources & $\mathbf{L}$ & $10 \%$ & & & & \\
\hline \multirow{8}{*}{ Function } & \multirow{8}{*}{$8(6 \%)$} & \multirow{8}{*}{4} & $\begin{array}{l}9.01 \text { childhood only happens once (Missed childhood) - } \\
\text { think childhood will last longer }\end{array}$ & H & $80 \%$ & \multirow{8}{*}{$8(6 \%)$} & \multirow{8}{*}{6} & \multirow{8}{*}{ Storing } & \multirow{8}{*}{ Reuse } \\
\hline & & & $\begin{array}{l}8.04 \text { stress will impact responsibilities and priorities - no } \\
\text { barriers to succeeding with priorities }\end{array}$ & $\mathbf{H}$ & $70 \%$ & & & & \\
\hline & & & 3.02 Still has functionality - waste & $\mathbf{H}$ & $90 \%$ & & & & \\
\hline & & & 6.1 first option - to dispose or recycle & H & $100 \%$ & & & & \\
\hline & & & 8.02 Thave to manage my time - I have plenty of time & I & $60 \%$ & & & & \\
\hline & & & $\begin{array}{l}11.02 \text { fear of change (more workttime) - its easier (less } \\
\text { work'time] (R) }\end{array}$ & $\mathbf{L}$ & $20 \%$ & & & & \\
\hline & & & $\begin{array}{l}11.03 \text { better outcome when consider more options - } \\
\text { better the devil you know }[R]\end{array}$ & $\mathbf{L}$ & $20 \%$ & & & & \\
\hline & & & 6.05 learnt system (easier) - slow & $\mathbf{L}$ & $50 \%$ & & & & \\
\hline \multirow{3}{*}{ Income } & \multirow{3}{*}{$3(2 \%)$} & \multirow{3}{*}{9} & 8.03 family priorities are important - selfishness & H & $70 \%$ & \multirow{3}{*}{$3(2 \%)$} & \multirow{3}{*}{12} & \multirow{8}{*}{ Purchasing refurbished } & \multirow{8}{*}{ Refurbish } \\
\hline & & & 5.08 got a mortgage to pay - not meeting mortgage & $\mathbf{I}$ & $70 \%$ & & & & \\
\hline & & & $\begin{array}{l}4.01 \text { family is the most important thing we have - no } \\
\text { regrets for living life }\end{array}$ & $\mathbf{I}$ & $40 \%$ & & & & \\
\hline \multirow{5}{*}{ Knowledge } & \multirow{3}{*}{$3(2 \%)$} & \multirow{3}{*}{9} & 3.1 helps to visualise outcome - unclear outcome & I & $70 \%$ & \multirow{3}{*}{$3(2 \%)$} & \multirow{3}{*}{12} & & \\
\hline & & & 6.03 piece of mind - antiety & $\mathbf{L}$ & $30 \%$ & & & & \\
\hline & & & 6.04 security - life threatening & $\mathbf{I}$ & $60 \%$ & & & & \\
\hline & & & 4.13 not waste of time - ease of use & $\mathbf{H}$ & $70 \%$ & & & & \\
\hline & & & 3.06 good to reuse if it can - unclear outcomes to the & H & $90 \%$ & & & & \\
\hline
\end{tabular}




\begin{tabular}{|c|c|c|c|c|c|c|c|c|c|}
\hline \multirow[t]{3}{*}{ Perception } & \multirow[t]{3}{*}{$5(4 \%)$} & \multirow[t]{3}{*}{7} & 4.11 Useful (limited) - Not useful & $\mathbf{I}$ & $50 \%$ & \multirow[t]{3}{*}{$5(4 \%)$} & \multirow[t]{3}{*}{9} & & \\
\hline & & & 5.04 not purchasing new - purchasing new (unnecessary) & I & $70 \%$ & & & & \\
\hline & & & 5.13 no concerned about status - status $(\mathrm{R})$ & $\mathbf{L}$ & $60 \%$ & & & & \\
\hline \multirow{4}{*}{ Price } & \multirow{4}{*}{$4(3 \%)$} & \multirow{4}{*}{8} & 9.02 not being able to pay bills - Spending what you don't & H & $80 \%$ & \multirow{4}{*}{$4(3 \%)$} & \multirow{4}{*}{10} & & \\
\hline & & & 9.07 not spending excess money - spending & $\mathbf{H}$ & $80 \%$ & & & & \\
\hline & & & 9.06 budget - spending & $\mathbf{I}$ & $50 \%$ & & & & \\
\hline & & & 7.05 I like to enioy life - I don't like dying (R) & $\mathbf{L}$ & $40 \%$ & & & & \\
\hline \multirow{7}{*}{ R_Value } & \multirow{7}{*}{$7(5 \%)$} & \multirow{7}{*}{5} & 9.08 saving money - spending & H & $90 \%$ & \multirow{7}{*}{$7(6 \%)$} & \multirow{7}{*}{7} & \multirow{8}{*}{ Storing } & \multirow{8}{*}{ Reuse } \\
\hline & & & 2.05 savvy - stupid & H & $80 \%$ & & & & \\
\hline & & & 9.03 llike a bargain - luxury & H & $90 \%$ & & & & \\
\hline & & & 9.04 I found best deal for my budget - buy first thing you & I & $60 \%$ & & & & \\
\hline & & & 2.01 depreciation - limited finances $(\mathrm{R})$ & $\mathbf{L}$ & $20 \%$ & & & & \\
\hline & & & 7.01 forces to budget - unlimited disposable income & $\mathbf{L}$ & $20 \%$ & & & & \\
\hline & & & 2.13 unsentimental - sentimental ( $R$ ) & $\mathbf{L}$ & $20 \%$ & & & & \\
\hline Size & $1(1 \%)$ & 13 & 9.06 budget - spending & I & $50 \%$ & $1(1 \%)$ & 16 & & \\
\hline \multirow{24}{*}{ Social } & \multirow{24}{*}{$27(21 \%)$} & & $\begin{array}{l}10.07 \text { does not matter they are not ideal, still good - it } \\
\text { would be bad }\end{array}$ & H & $90 \%$ & \multirow{14}{*}{$14(11 \%)$} & \multirow{14}{*}{2} & & \\
\hline & & & $\begin{array}{l}10.06 \text { I want to live in a clean place - wanting to live in a } \\
\text { dirty place }\end{array}$ & H & $90 \%$ & & & & \\
\hline & & & 2.04 role model-destructive & H & $80 \%$ & & & & \\
\hline & & & 10.05 people should do the right thing - doing wrong & H & $80 \%$ & & & & \\
\hline & & & 5.05 builds a better future - restricts building the future & $\mathbf{I}$ & $80 \%$ & & & & \\
\hline & & & 1.03 its good - it changes things $(\mathrm{R})$ & $\mathbf{I}$ & $80 \%$ & & & & \\
\hline & & & 8.06 that's what your judged by - just forgotten & H & $70 \%$ & & & Engagement & Recucle \\
\hline & & & 1.07 I'm contributing - is unknown & $\mathbf{I}$ & $70 \%$ & & & & \\
\hline & & & 2.02 so my children have something - because its & I & $70 \%$ & & & & \\
\hline & & & 9.1 want them to have same life style - poorer lifestyle & I & $70 \%$ & & & & \\
\hline & & & 8.08 no effect - impacts on my family $(R)$ & I & $60 \%$ & & & & \\
\hline & & & 6.06 growth for future generations - scarce resources & I & $50 \%$ & & & & \\
\hline & & & 1.06 because I want to be good - like to be realistic & $\mathbf{L}$ & $50 \%$ & & & & \\
\hline & & & $\begin{array}{l}8.05 \text { I want there to be a future for my children - we leave } \\
\text { an unsustainable environment }\end{array}$ & $\mathbf{L}$ & $50 \%$ & & & & \\
\hline & & 2 & 1.05 needs meaning - so I won't die & H & $90 \%$ & & & & \\
\hline & & & 10.1 I want to feel safe in the world l live in - feeling unsafe & H & $80 \%$ & & & & \\
\hline & & & 2.08 its protective - its non protective & I & $70 \%$ & & & & \\
\hline & & & 10.01 I want to be a good example - I would be a bad & I & $70 \%$ & & & & \\
\hline & & & $\begin{array}{l}10.02 \text { because l care about the future - you don't care } \\
\text { about the future }\end{array}$ & I & $70 \%$ & $9(7 \%)$ & 4 & On giving & Reuse \\
\hline & & & $\begin{array}{l}10.03 \text { I want people to be good people - I want people to } \\
\text { be bad }\end{array}$ & 1 & $70 \%$ & & & & \\
\hline & & & 1.01 continued existence - unknown own & $\mathbf{I}$ & $80 \%$ & & & & \\
\hline & & & 5.07 someone else benefits - no one benefits & H & $90 \%$ & & & & \\
\hline & & & 2.1 self esteem - poor self esteem & $\mathbf{I}$ & $70 \%$ & & & & \\
\hline & & & $\begin{array}{l}10.09 \text { I want to meet my own expectations - being a no } \\
\text { hoper }\end{array}$ & H & $90 \%$ & & & & \\
\hline
\end{tabular}




\begin{tabular}{|c|c|c|c|c|c|c|c|c|c|}
\hline & & & $\begin{array}{l}12.05 \text { if someone could use it they should - not finding } \\
\text { out if mobile phone can be useful for somebody else }\end{array}$ & I & $90 \%$ & \multirow{3}{*}{$4(3 \%)$} & \multirow{3}{*}{10} & \multirow{3}{*}{ Purchasing refurbished } & \multirow{3}{*}{ Refurbish } \\
\hline & & & $\begin{array}{l}10.04 \text { I want a safe world to live in - an unsafe world to live } \\
\text { in }\end{array}$ & $\mathbf{L}$ & $60 \%$ & & & & \\
\hline & & & 1.02 because I care - reality $(R)$ & $\mathbf{L}$ & $50 \%$ & & & & \\
\hline \multirow{6}{*}{ Spare } & \multirow{6}{*}{$6(5 \%)$} & \multirow{6}{*}{6} & $\begin{array}{l}7.08 \text { you can enjoy dismantling - you can no longer enjoy } \\
\text { dismantling it ( } \mathrm{R} \text { ) }\end{array}$ & H & $80 \%$ & \multirow{6}{*}{$6(5 \%)$} & \multirow{6}{*}{8} & \multirow{6}{*}{ Storing } & \multirow{6}{*}{ Reuse } \\
\hline & & & $\begin{array}{l}7.1 \text { enjoy repurposing existing components - dislike } \\
\text { repurposing components }\end{array}$ & H & $80 \%$ & & & & \\
\hline & & & $\begin{array}{l}7.02 \text { make different things out of it (enjoy) - make the } \\
\text { same thing out of it }\end{array}$ & $\mathbf{H}$ & $100 \%$ & & & & \\
\hline & & & 7.03 does not go to waste - goes to waste & $\mathbf{H}$ & $100 \%$ & & & & \\
\hline & & & 7.07 Ilove it - I hate it & I & $60 \%$ & & & & \\
\hline & & & 7.06 Idon't like to be stressed - l like to be stressed & $\mathbf{L}$ & $40 \%$ & & & & \\
\hline \multirow{9}{*}{ Age of technology } & \multirow{9}{*}{$9(7 \%)$} & \multirow{9}{*}{3} & 2.11 sustainability - interdependence & $\mathbf{H}$ & $80 \%$ & \multirow{9}{*}{$9(7 \%)$} & \multirow{9}{*}{4} & \multirow{9}{*}{ Purchasing refurbished } & \multirow{9}{*}{ Refurbish } \\
\hline & & & 4.12 saves time - give up & H & $70 \%$ & & & & \\
\hline & & & 8.12 total reliance - nice not to rely on it & H & $70 \%$ & & & & \\
\hline & & & 11.09 Self acknowledgement - no acknowledgement & H & $80 \%$ & & & & \\
\hline & & & $\begin{array}{l}2.12 \text { not interested in latest device - invest time in other } \\
\text { activities }\end{array}$ & H & $80 \%$ & & & & \\
\hline & & & $\begin{array}{l}5.1 \text { increasing lifespan of parts - longevityllifespan - } \\
\text { breaking (R) }\end{array}$ & I & $70 \%$ & & & & \\
\hline & & & $\begin{array}{l}12.1 \text { need can be satisfied by second hand phone - get all } \\
\text { the extra features (but don't need) }\end{array}$ & $\mathbf{I}$ & $90 \%$ & & & & \\
\hline & & & $\begin{array}{l}12.06 \text { match price and tech with what I NEED - just what } \\
\text { you want regardless of cost }\end{array}$ & $\mathbf{L}$ & $60 \%$ & & & & \\
\hline & & & $\begin{array}{l}5.11 \text { keeps you up with the play - not being able top } \\
\text { partake in new tech }\end{array}$ & $\mathbf{I}$ & $70 \%$ & & & & \\
\hline Trust [data] & $1(1 \%)$ & 13 & 8.11 it affects family - no effect & I & $60 \%$ & $1(1 \%)$ & 16 & Ongiving & Reuse \\
\hline
\end{tabular}


3) Group - Word (Content) cloud

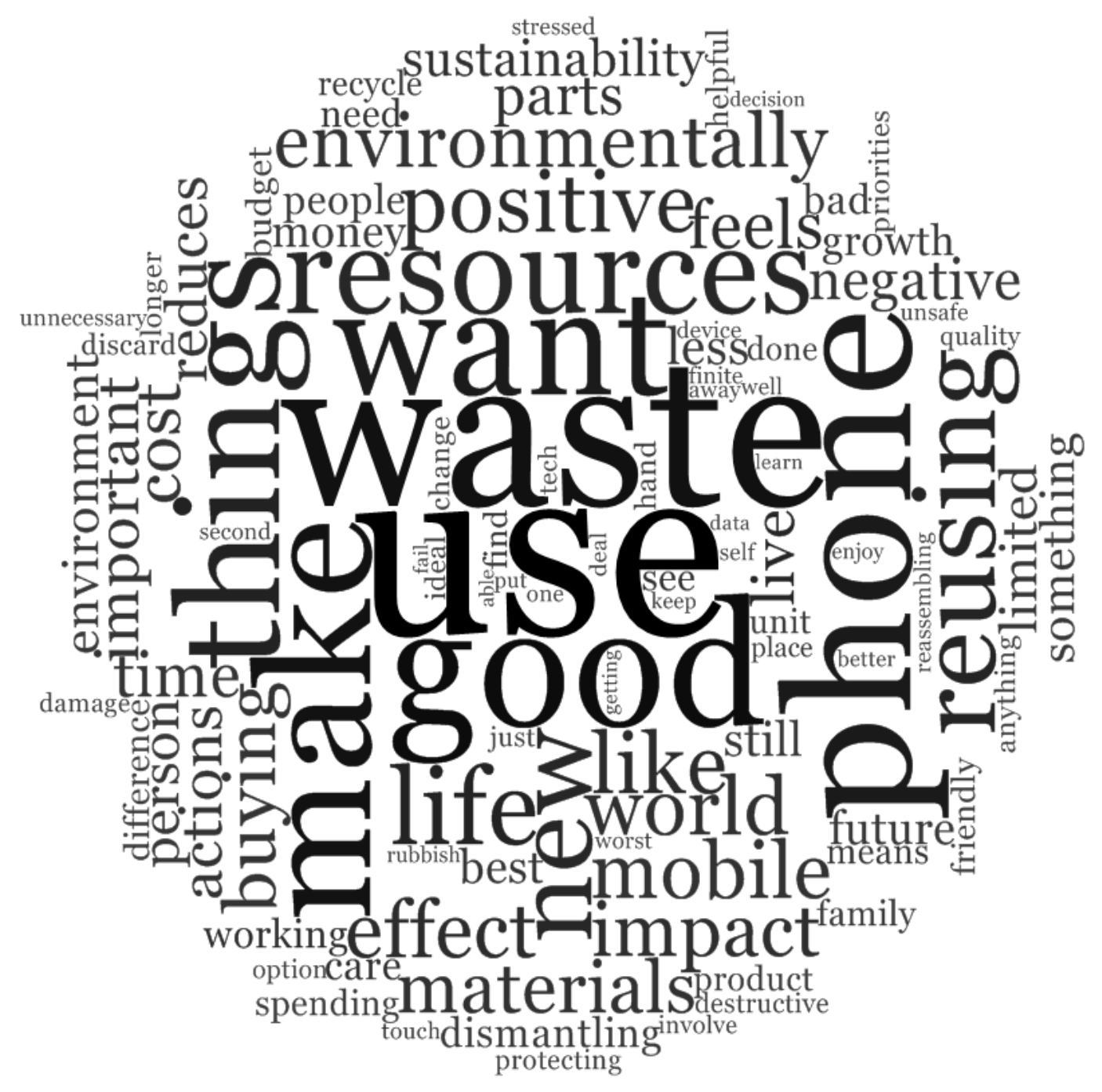


4) Human ethics

a) Information sheet

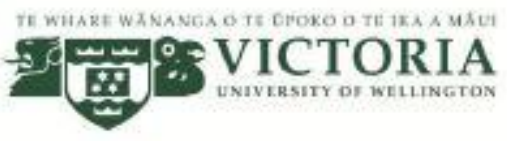

\section{Consumer attitudes towards the sustainable management of used mobile phones}

\section{INFORMATION SHEET FOR PARTICIPANTS}

Thank you for your interest in this project. Please read this information before deciding whether or not to take part. If you decide to participate, thank you. If you decide not to take part, thank you for considering my request.

Who am I?

My name is Philip Coffey and I am a Masters student in The School of Information Management at Victoria University of Wellington. This research project is work towards my thesis.

What is the aim of the project?

Mobile phones are very pervasive in today's society and what people choose to do with used mobile dispose has a direct impact on the natural environment. The purpose of this research project is to study people's attitudes towards managing mobile phones when they are perceived to have reached the end of their useful lives. This research has been approved by the Victoria University of Wellington Human Ethics Committee [provide approval number].

How can you help?

If you agree to take part I will interview you in a place that is convenient for you but it will need to be quite to enable you to concentrate. The interview will consist of five background questions and an elicitation process where you will be asked to describe in a series of contrasting statements how you feel about managing used mobile phones. During this process you may be asked a series of "why" or "how" questions to clarify some of the statements produced. You will then be asked to rate these contrasting statements on a 5 point scale.

The interview will take approximately 60 minutes. I will audio record the interview and may transcribe it to assist with analysis. You can stop the interview at any time without giving a reason. You can withdraw from the study up to four weeks after the interview. If you withdraw, the information you provided will be destroyed or returned to you.

What will happen to the information you give?

This research is confidential. I will not name you in any reports, and I will not include any information that would identify you. Only my supervisors and I will read the notes or any transcripts of the interview. All interview transcripts, summaries and any recordings will be kept securely and destroyed 3 years after the research ends. 
b) Background questions

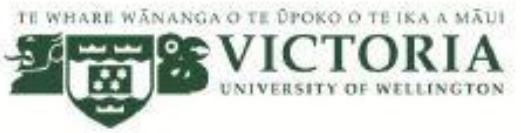

\section{Consumer attitudes towards the sustainable management of used mobile phones}

\section{Background questions}

Name:

1. What is your age?

$18-34$

$35-55$

$56-73$

$74-91$

2. What is your income range?

$\$ 0-\$ 25,000$

$\$ 25,000-\$ 50,000$

$\$ 50,000-\$ 75,000$

$\$ 75,000-\$ 100,000$

Above $\$ 100,000$

3. What is your highest education level?
Postgraduate
Undergraduate
High school
Other
If other please specify: 
4. What is your current employment?

Full time employed

Part time employed

Self employed

Student

Retired

Unemployed

Other

If other please specify:

5. If you are employed, what is your current role?

Please specify: 
Informed consent

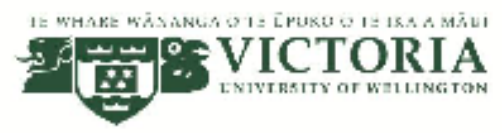

\section{Consumer attitudes towards the sustainable management of used mobile phones \\ CONSENT TO INTERVIEW}

This consent form will be held for 3 years.

Researcher: Philip Coffey, School of Information Management, Victoria University of Wellington.

- I have read the Information Sheet and the project has been explained to me. My questions have been answered to my satisfaction. I understand that I can ask further questions at any time.

- I agree to take part in an audio recorded interview.

I understand that:

- I may withdraw from this study up to four weeks after the interview and any information that I have provided will be returned to me or destroyed.

- The information I have provided will be destroyed 3 years after the research is finished.

- Any information I provide will be kept confidential to the researcher and the supervisor. I understand that the results will be used for a Masters report and a summary of the results may be used in academic reports and/or presented at conferences.

- My name will not be used in reports, nor will any information that would identify me.

- I would like a copy of the interview repertory grid and interview Yes No transcript (if transcribed):

- I would like to receive a copy of the final report and have added my Yes No email address below.

Signature of participant:

Name of participant:

Date:

Contact details: 